\title{
27. LATE MIOCENE-HOLOCENE PALEOCEANOGRAPHY OF THE WESTERN EQUATORIAL ATLANTIC: EVIDENCE FROM DEEP-SEA BENTHIC FORAMINIFERS ${ }^{1}$
}

\author{
Hisato Yasuda ${ }^{2}$
}

\begin{abstract}
Changes in deep-water environments of the western Atlantic over the past six million years were investigated by studying benthic foraminiferal abundance changes in 335 samples from Sites $926(3598 \mathrm{~m}), 928(4010 \mathrm{~m})$, and $929(4358 \mathrm{~m})$ on the Ceara Rise. The location of these sites on a transect at lower abyssal depths provides a unique opportunity to evaluate changes in water mass properties as well as in the supply of organic matter from the surface waters, especially because other Leg 154 studies provided a precise time scale for correlations between the sites.

Relative abundances and fluxes of the most abundant species were calculated, with emphasis on the four most common species-Nuttallides umbonifera, Globocassidulina subglobosa, Epistominella exigua, and Alabaminella weddellensis. There are two patterns in relative abundance of these species with time: the first group of species (N. umbonifera and G. subglobosa) shows abundance variations that differ from site to site (i.e., with depth). The other group (E. exigua and A. weddellensis) shows variations in relative abundance that are similar at all three sites and thus independent of depth.

In the first group, G. subglobosa increased in relative abundance at the shallowest Site 926 from 2.4 to $1 \mathrm{Ma}$, but decreased at the two deeper sites. In contrast, $N$. umbonifera, a marker species for corrosive bottom waters and specifically for Antarctic Bottom Water (AABW), shows large fluctuations in relative abundance, but overall increased in relative abundance at the two deeper sites and decreased at the shallower site from 2.1 Ma to the present. Its relative abundance shows three peaks during the last 0.9 m.y., which were coeval at the two deeper sites. These large fluctuations in abundance of $N$. umbonifera suggest that production of AABW fluctuated in intensity, with increases during interglacial periods. AABW formation increased overall from about $3 \mathrm{Ma}$, and the amplitude of the fluctuations increased from $0.9 \mathrm{Ma}$ to present. The differences between sites are interpreted as showing that the deeper Sites 928 and 929 were commonly within AABW, whereas the shallower site was not.

The species in the second group, E. exigua and A. weddellensis, occur in the recent oceans commonly in oceanic regions where spring blooms lead to seasonal deposition of phytodetrital material. At the Ceara Rise sites, they show variations in relative abundance that are similar at all three sites from 4.5 to $1.2 \mathrm{Ma}$, confirming that these species react to environmental factors that are not depth related, and thus possibly to surface productivity. The two species, however, do not covary exactly: at $2.6 \mathrm{Ma}$, for example, E. exigua decreased in relative abundance whereas $A$. weddellensis increased.
\end{abstract}

\section{INTRODUCTION}

Stratigraphic changes in deep-sea benthic foraminiferal faunas have been commonly associated with changes in North Atlantic deep-water circulation during the Pleistocene glacial-interglacial cycles (Streeter, 1973; Streeter and Shackleton, 1979; Schnitker, 1974, 1979, 1980; Peterson and Lohmann, 1982). Studies on recent benthic foraminiferal assemblages likewise revealed a relation between faunal composition and hydrographic parameters such as temperature, salinity, oxygen content, and carbonate saturation (Murray, 1973; Lohmann, 1978; Corliss, 1979; Corliss and Honjo, 1981; Peterson, 1984; Mead, 1985). Many different authors have attempted to correlate the distribution of deep-water benthic foraminifers with changes in water mass circulation patterns in the deep oceans, and benthic foraminiferal faunal changes have been linked to changes in stable isotope records (e.g., Woodruff and Savin, 1989; Boersma, 1990; Hermelin, 1991; Thomas, 1992).

On the other hand, several authors reported that the abundance of several species of benthic foraminifers is correlated to surface primary productivity and thus the availability of food on the seafloor. Much data on faunas along continental margins suggest that faunas rich in genera such as Melonis, Uvigerina, and Bolivina reflect a high food supply rather than oxygen concentration in the bottom waters (e.g., Lutze and Coulbourn, 1984; Mackensen et al., 1985; Altenbach and

${ }^{1}$ Shackleton, N.J., Curry, W.B., Richter, C., and Bralower, T.J. (Eds.), 1997. Proc. ODP, Sci. Results, 154: College Station, TX (Ocean Drilling Program).

22-5-1 Akebono-machi, Department of Geology, Kochi Univeristy, Kochi 780, Japan. yasuda@cc.kochi-u.ac.jp
Sarnthein, 1989; Schnitker, 1993; Rathburn and Corliss, 1994; Corliss, 1983; Miao and Thunell, 1993; Hermelin and Shimmield, 1990; Thomas et al., 1995).

Other species of benthic foraminifers have been observed to respond opportunistically to a seasonal supply of phytodetritus under overall oligotrophic circumstances in the open ocean (Gooday, 1988, 1993; Gooday and Lambshead, 1989). High rates of phytodetritus deposition can not be linked in a simple way to high surface productivity, but reflect hydrographic conditions in the upper layers of the oceans (particularly, a deep layer of winter mixing), that lead to a strong phytoplankton bloom (Campbell and Aarup, 1992). The opportunistic, phytodetritus-exploiting species have been used to reconstruct the occurrence of episodes with common phytodetritus deposition in down-core investigations (Smart et al., 1994; Thomas et al., 1995; Thomas and Gooday, in press).

Benthic foraminiferal faunas thus can play an important role in the investigation of deep-water circulation patterns as well as paleoproductivity (e.g., Mackensen et al., 1995). The purpose of this study is to examine the long-term changes in deep-water environments as based on benthic foraminiferal faunal and abundance changes during the past 6 m.y.

\section{MATERIAL AND METHODS}

Samples were taken from three sites drilled on Leg 154 on the Ceara Rise (equatorial Atlantic Ocean) on a depth transect between 3000 and $4300 \mathrm{~m}$, at lower abyssal depths (Fig. 1). Hole 926A $\left(3^{\circ} 43.146^{\prime} \mathrm{N}, 43^{\circ} 44.884^{\prime} \mathrm{W}, 3598.4 \mathrm{~m}\right)$ is presently in the lower part of North Atlantic Deep Water (NADW). Hole 928A $\left(5^{\circ} 27.320^{\prime} \mathrm{N}\right.$, 
$42^{\circ} 54.489^{\prime} \mathrm{W}, 4010.7 \mathrm{~m}$ ) is under the mixing zone of NADW and Antarctic Bottom Water (AABW), and is near the lysocline. Hole 929A $\left(5^{\circ} 58.573^{\prime} \mathrm{N}, 43^{\circ} 44.396^{\prime} \mathrm{W}, 4357.6 \mathrm{~m}\right)$ is presently under AABW.

Samples were taken at $1.5-\mathrm{m}$ intervals (one sample per section) over a sediment section corresponding to the last 6-7 m.y.; 114 samples from Hole 926A, 107 samples from 928A, and 114 samples from Hole 929A were examined (Tables 1-3). Samples of about $10 \mathrm{~cm}^{3}$ were treated with a $1 \%-3 \%$ hydrogen peroxide solution for $1-12 \mathrm{hr}$, and wet-sieved over a $63-\mu \mathrm{m}$ screen. The coarse fraction was dried and split into aliquots containing approximately 200-300 specimens. More than 200 specimens were identified and counted. Some samples at Site 929 did not contain enough specimens or carbonate fragments at all. Some miliolid and unilocular species were not identified to the generic and specific level because of their rarity and extremely high diversity. The preservation of specimens was almost good, except for several samples at Site 929 that had completely dissolved.

The age models in this paper are based on the correlation between magnetic susceptibility records and previously published isotope records (Curry, Shackleton, Richter, et al., 1995). Benthic foraminiferal accumulation rates were estimated by using gamma-ray attenuation porosity evaluator (GRAPE) density data and sedimentation rates as derived from these age models (Tables $4-6$, back pocket). The benthic foraminiferal accumulation rate (BFAR, the number of foraminifers per $\mathrm{cm}^{2}$ per $1000 \mathrm{yr}$ ) is defined as follows:

$$
\mathrm{BFAR}=\# \mathrm{BF} / \mathrm{g} \times \mathrm{GRD}\left(\mathrm{g} / \mathrm{cm}^{3}\right) \times \operatorname{SDR}(\mathrm{cm} / \mathrm{ky}),
$$

in which \#BF is the number of benthic foraminifers, GRD is the GRAPE density, and SDR is the sedimentation rate. The BFAR has been used as a proxy for deposition rate of organic matter to the sea floor, and of primary productivity (Berger and Diester-Haass, 1988; Herguera and Berger, 1991; Herguera, 1992).

To observe long-term trends of benthic foraminiferal abundances, 3-point moving averages were calculated for the relative abundances and accumulation rates of the four most abundant species, the phytodetritus species, and the total number of individuals.

\section{RESULTS Relative Abundances}

The relative abundances of the most common species at all three sites were plotted vs. numerical age in Figures 2-4. The four most abundant species (Alabaminella weddellensis, Epistominella exigua, Globocassidulina subglobosa, and Nuttallides umbonifera) together account for about $60 \%$ of the fauna in most samples. These species are the most common and cosmopolitan lower abyssal species in the present world oceans since the early-middle Miocene (Woodruff and Savin, 1989), and the fluctuations in their relative and absolute abundance can thus give information on changes in the abyssal paleoenvironment. The relative abundances of these four species for each sites are shown versus numerical age in Figure 5, with superimposed 3point moving averages; data for all sites together are shown in Figure 6.

Globocassidulina subglobosa shows different fluctuations in relative abundance at all sites, although the records for the two deeper sites show some similarities - at both these sites the relative abundance decreases overall since about 4.5 Ma. At the shallower site it fluctuates, but appears to have high values between 2.5 and $1.0 \mathrm{Ma}$, and during the last 0.5 m.y.

The relative abundance of $N$. umbonifera also shows different patterns by depth, especially during the last 3 m.y. At the shallowest site, Site 926, it shows generally weak fluctuations throughout the last 6 m.y., possibly with a peak abundance at $4.5 \mathrm{Ma}$. At the two deeper sites, the relative abundance of this species shows similar patterns, with the lowest values between 3 and $4.5 \mathrm{Ma}$, and very strong fluctu- ations superimposed on a relative increase over the last 3 m.y. The species shows peaks in abundance at both deeper sites at about 2.5 $\mathrm{Ma}, 1.5 \mathrm{Ma}$, and three peaks during the last $1 \mathrm{~m} . \mathrm{y}$.

Alabaminella weddellensis is a very small species, and its taxonomy has been rather confused. The species has commonly been identified as Eponides pusillus or Eilohedra weddellensis in samples from the Pacific Ocean (Loeblich and Tappan, 1994). Specimens from both the Atlantic and the Pacific were studied, and a conclusion was reached that they belong to the same species. Because A. weddellensis is a commonly used name in studies of Atlantic foraminifers, it has also been applied to this specimen. The patterns of fluctuation in relative abundance of this species are very similar at all three sites, especially during the last 3.5 m.y. The species shows peak abundances at about 3.5-3.2 Ma, then a decline followed by a rapid increase to peak values at 2.6-2.0 Ma, then another decrease followed by a peak at $1.2-0.9 \mathrm{Ma}$, and a final increase from $0.7 \mathrm{Ma}$ on.

Epistominella exigua also has similar patterns of fluctuations in relative abundance at all three sites. Low values occurred at about 5.5, 4.8 and 4.2 Ma, but stronger fluctuations occurred after about 3.5 Ma. High values occurred between about 3.2 and $2.6 \mathrm{Ma}$ (when $A$. weddellensis had low abundances), followed by an abrupt decline at about 2.6 Ma, when A. weddellensis increased. After 2.6 Ma the species fluctuated in relative abundance, with on overall increase at all sites since about $0.7 \mathrm{Ma}$.

Epistominella exigua, A. weddellensis and a few other species have been observed to inhabit phytodetrital aggregates, and the distribution and abundance of these species has been said to be controlled by the occurrence of strongly seasonal fluxes of this material (Gooday, 1988, 1993). Summed relative abundances of these two taxa at all three sites are shown in Figure 7A. In addition, the summed relative abundance of these two species is shown together with that of two other species that resemble them morphologically; i.e., they are small, trochospiral, and have thin and translucent walls. The two additional species are Epistominella sp. A (an obese variant of E. exigua) and Ioanella tumidula (Figure 7B). Figures 7A and 7B are similar because E. exigua and A. weddellensis are much more common than the other two taxa, although the resemblance between the curves for the different sites is largest in Figure 7B.

The phytodetritus species show a low in relative abundance at about 5.4 Ma and another at around 4.1-4.2 Ma. These show increased amplitude fluctuations from about $3 \mathrm{Ma}$ on, and even more extreme after 1.5 Ma. A sharp drop occurred at around 0.7 Ma. During the last 0.3 m.y., their abundances decreased at the shallowest and the deepest sites, but increased at the middle Site 928.

Several species show first or last appearances at all three sites during the studied interval (Figure 8). Pleurostomella spp. decreased in relative abundance after about $3 \mathrm{Ma}$ and had their last occurrence at $2 \mathrm{Ma}$ at the deepest Site 929, at 1.6 Ma at the middle Site 928, and at 1.3 Ma at Site 926. Stainforthia fusiformis first appeared at about 4.0 $\mathrm{Ma}$ at the deeper two sites. Pullenia osloensis had a peak in abundance at about $3 \mathrm{Ma}$, and abruptly decreased in abundance at about $2.6 \mathrm{Ma}$ at Sites 926 and 928. Ioanella tumidula shows similar fluctuations in abundance at all three sites, but was more common at the shallower Site 926.

\section{Benthic Foraminiferal Accumulation Rates}

Accumulation rates of benthic foraminifers are caused by complex multiple factors, which include changes in the deposition rate of particulate organic matter to the seafloor (which in turn may be caused by changes in productivity of organic matter in the surface waters, changes in export productivity, changes in the rate of oxidation of organic matter in the water column, and other factors, as well as the water depth at the site). In addition, physicochemical parameters of sea water in the region may be a factor. Furthermore, dissolution may influence the preservation of specimens. 

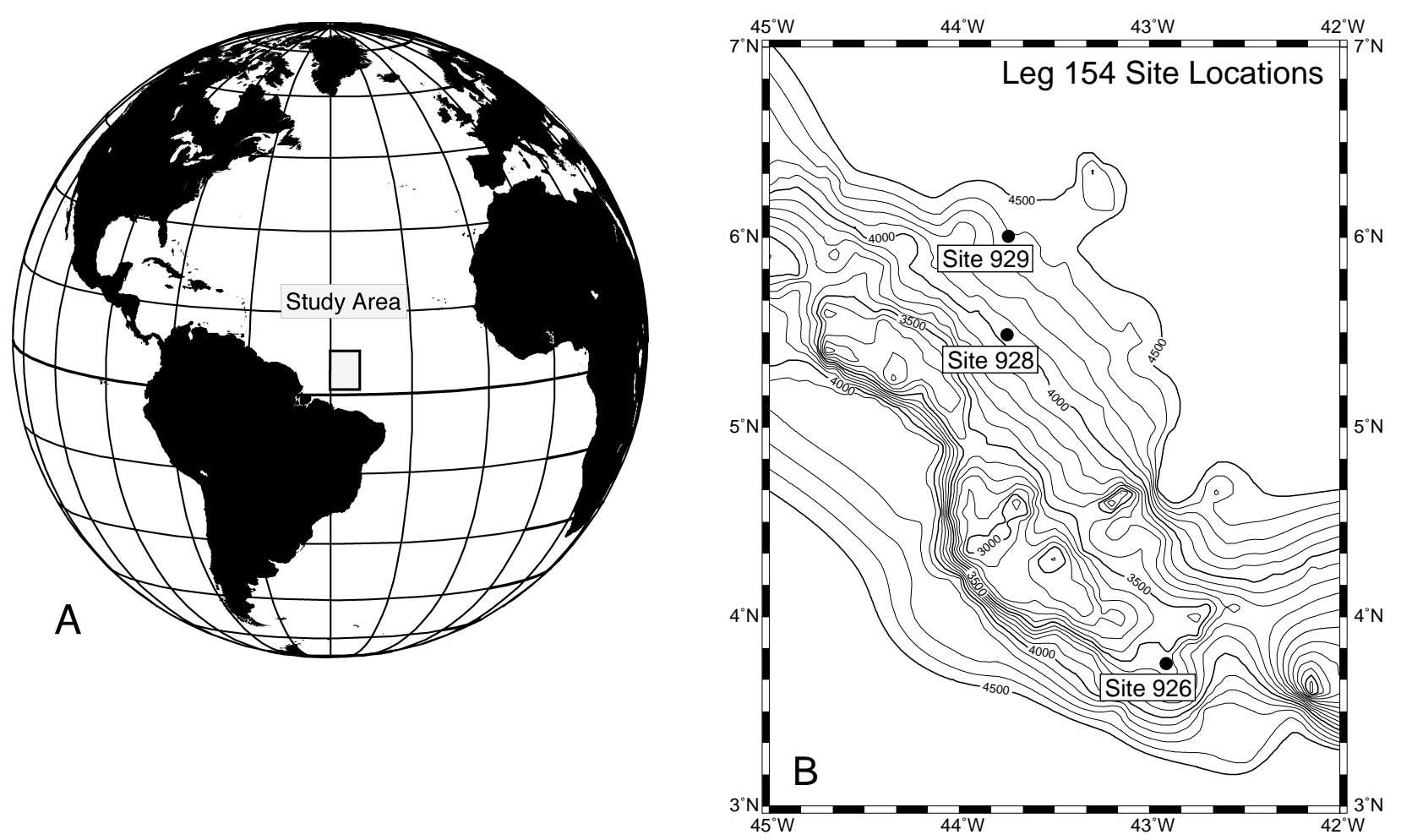

Figure 1. A. Location map of the Ceara Rise in the equatorial Atlantic Ocean. B. Position of Sites 926, 928, and 929 on the Ceara Rise.

Fluctuations in the accumulation rates of E. exigua and N. umbonifera are of greater amplitude than those of $G$. subglobosa and $A$. weddellensis (Figs. 9, 10). All species show a resemblance between the curves for relative abundance and accumulation rate (Figs. 6, 10). The fluctuations in accumulation rates of $N$. umbonifera were largest during the last 3 m.y. Before that time, the accumulation rate of this species was similar at all three sites, and afterwards it was more abundant, with larger fluctuations at the two deepest sites. Three peaks in abundance occurred in the last 1 m.y.

Accumulation rates of A. weddellensis increased at about 3.5 Ma, with very similar patterns of fluctuation at all three sites. Epistominella exigua shows strong fluctuations in accumulation rates after about 3.2 Ma, especially at Site 929. Globocassidulina subglobosa shows similar rates of accumulation at Sites 926 and 928 before about 4.4 Ma. After about 3.0 Ma, the species generally decreased in accumulation rate at the two deeper sites, with a maximum at the shallower site at about $2.2 \mathrm{Ma}$.

The phytodetritus group of species shows very strong fluctuations in accumulation rates over time, with the largest amplitude at the two deepest sites. Fluctuations have a larger amplitude than those in relative abundance (Figs. 7, 11). Before $4 \mathrm{Ma}$ the accumulation rates were very low at Site 929 , with higher, co-varying values at the two shallower sites. Overall, accumulation rates increased from $4 \mathrm{Ma}$, but there was a large decrease at all three sites at about $2 \mathrm{Ma}$, until about 1.6 Ma. The fluctuations in accumulation rate occur at similar times at all three sites, and there is no obvious relation between the depths of the sites and the accumulation rates at any point in time.

Accumulation rates of the total fauna are shown in Figures 12 and 13. There is a strong correlation between the accumulation rate of the total fauna and that of the phytodetritus species (Figs. 11, 14).

Before 3.4 Ma, the accumulation rates were very low at the deepest Site 929, at the time when carbonate accumulation rates were also low (Curry, Shackleton, Richter, et al., 1995). Accumulation rates at both shallower sites were very similar, with a high at about $5.2 \mathrm{Ma}$, and a low at 4.2 Ma. At about 3.2 Ma the accumulation rates increased strongly, as did the amplitude of their fluctuations. The lower values of the last 3.2 m.y. are similar to the average values before 3.2 Ma. Peaks occurred synchronously at all three sites, with the highest peak at about 2.1 Ma. Accumulation rates would be expected to be higher at the shallower site (Herguera and Berger, 1991), but this is not observed consistently.

\section{DISCUSSION}

The observed changes in deep-sea benthic foraminiferal faunas have been caused by a combination of changes in deep-water circulation, deep-water chemical properties, and productivity, as described for the modern ocean by Mackensen et al. (1995). The four most common species have very similar abundances at the three sites between 3 and 3.4 Ma, suggesting that the deep-water environments at all sites were similar at these times, and thus that waters at these depths were not strongly stratified.

The benthic foraminiferal accumulation rate (BFAR) is controlled mostly by a limited food supply, and additionally due to local chemical conditions plus differences of preservation due to carbonate saturation. Accordingly, the BFAR are possibly a recorder of surface paleo-productivity, if carbonate dissolution and water mass changes are also taken into account through the interval. Herguera (1992) researched a relation between the BFAR and the decay of organic carbon with depth, and concluded that the BFAR is directly linked to the flux of organic matter to the seafloor.

Comparison of the BFAR at Sites 926, 928, and 929 (Figs. 12, 13) clearly shows a major difference between faunas before and after $\sim 3.2 \mathrm{Ma}$ (with a short exception from 3.8 to $4.0 \mathrm{Ma}$ ). Before 3.2 Ma, the BFAR was much lower at the deepest site than at the two shallower ones, and there was much less difference than predicted by Herguera's equation between the BFAR at the two shallower sites. However, the BFAR at Site 929 was obviously not equal, which indicates that there was a big contrast in carbonate dissolution between water depths of 4000 and $4300 \mathrm{~m}$ before and after $\sim 3.2 \mathrm{Ma}$. At the deepest site $(4300 \mathrm{~m})$, most of the species have low accumulation rates. This 
Table 1. Census data for the benthic foraminifer species from Hole 926A in the $63-\mu \mathrm{m}$ fraction.

\begin{tabular}{|c|c|c|c|c|c|c|c|c|c|c|c|c|c|c|c|c|c|c|}
\hline $\begin{array}{l}\text { Core, section, } \\
\text { interval }(\mathrm{cm})\end{array}$ & $\begin{array}{l}\text { Depth } \\
(\mathrm{m})\end{array}$ & $\begin{array}{l}\text { Depth } \\
\text { (mcd) }\end{array}$ & $\begin{array}{l}\text { Age } \\
\text { (Ma) }\end{array}$ & 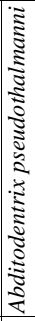 & 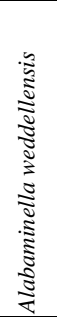 & 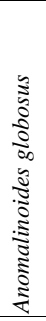 & & & 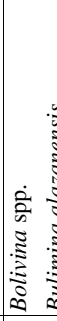 & 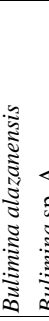 & 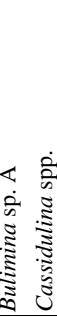 & 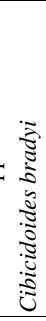 & 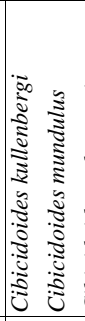 & & 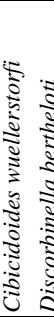 & & 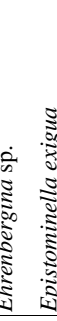 & 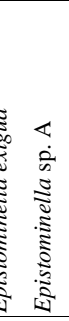 \\
\hline $\begin{array}{l}154-926 \mathrm{~A}- \\
1 \mathrm{H}-1.80-82\end{array}$ & & & & & & & & & & & & & & & & & & \\
\hline $\begin{array}{l}1 \mathrm{H}-1,80-82 \\
1 \mathrm{H}-2,80-82\end{array}$ & $\begin{array}{l}0.8 \\
2.3\end{array}$ & $\begin{array}{l}0.86 \\
2.36\end{array}$ & .073 & & $\begin{array}{l}68 \\
47\end{array}$ & & & & & & $\begin{array}{rl}13 & 1 \\
8 & 5\end{array}$ & 5 & $\begin{array}{l}1 \\
4\end{array}$ & & $\begin{array}{l}0 \\
7\end{array}$ & & & 10 \\
\hline $1 \mathrm{H}-3,29-3$ & 3.29 & 3.35 & 0.104 & 1 & 35 & & & 1 & 2 & & 75 & & 9 & & 2 & & & 12 \\
\hline $2 \mathrm{H}-1,80-82$ & 4.8 & 7.1 & 0.219 & & 80 & & & & & & 71 & & 6 & 1 & & & & \\
\hline $2 \mathrm{H}-2,80-82$ & 6.3 & 8.6 & 0.265 & & 32 & & & & 4 & & 62 & & 6 & & & & & 195 \\
\hline $2 \mathrm{H}-3,80-82$ & 7.8 & 10.1 & 0.311 & & 46 & & & & & & 12 & & 2 & & 1 & & & 276 \\
\hline $2 \mathrm{H}-4$ & 9.3 & 11.6 & 0.357 & & 56 & 1 & & & & & $\begin{array}{ll}9 & 1\end{array}$ & & 2 & & & & 12 & $25 \quad 5$ \\
\hline $2 \mathrm{H}-5$ & 10.8 & 13.1 & 0.403 & & 112 & 1 & & & & 21 & 11 & & 2 & & 1 & & & 4 \\
\hline $2 \mathrm{H}-6,8$ & 12.3 & 14.6 & 0.448 & & 58 & & & & & & 16 & & 3 & & 2 & & & $9 \quad 1$ \\
\hline $2 \mathrm{H}-7,20-22$ & 13.2 & 15.5 & 0.476 & & 48 & & & & 8 & & $4 \quad 1$ & & 47 & & 3 & & & 36 \\
\hline $3 \mathrm{H}-1,80-$ & 14.3 & 15.39 & 0.473 & & 62 & 1 & & & & & $\begin{array}{ll}7 & 3 \\
\end{array}$ & & 1 & & 3 & & & $8 \quad 1$ \\
\hline $3 \mathrm{H}-2$ & 15.8 & 16.89 & .518 & & 71 & & & & & & 51 & & 5 & & 3 & & & $\begin{array}{lll}37 & 12 \\
37\end{array}$ \\
\hline $3 \mathrm{H}-3$ & 17.3 & 18.39 & 0.564 & & 10 & & & & 1 & & 6 & 1 & 615 & & 2 & & & 9 \\
\hline $3 \mathrm{H}-4$ & 18.8 & 19.89 & 0.61 & & 59 & 1 & & & 1 & & $\begin{array}{ll}7 & 4 \\
\end{array}$ & & & & 5 & & & \\
\hline $3 \mathrm{H}-5,80-82$ & 20.3 & 21.39 & 0.655 & & 32 & & & & & & 123 & & 117 & & 3 & & & \\
\hline $3 \mathrm{H}-6,80-82$ & 1.8 & 22.89 & .701 & & 55 & & & 1 & 1 & & 71 & & 25 & & 1 & & & 262 \\
\hline $4 \mathrm{H}-1$ & 23.8 & 26.38 & 0.807 & & 64 & 1 & 1 & 1 & 1 & & 4 & & 523 & & 1 & & & 275 \\
\hline $4 \mathrm{H}-$ & 25.3 & 27.88 & .853 & & 45 & & & & 1 & & & & 64 & & 1 & & & $\begin{array}{ll}68 & 6\end{array}$ \\
\hline $4 \mathrm{~F}$ & 2 & 29.38 & 0.899 & & 50 & & & & 1 & & 6 & & 34 & & & & 12 & $29 \quad 2$ \\
\hline $4 \mathrm{H}-4,8$ & 28.3 & 30.88 & 0.944 & & 81 & & & & 1 & & $\begin{array}{lll}5 & 10\end{array}$ & & 110 & & 3 & & 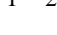 & 36 \\
\hline $4 \mathrm{H}-5,80-8$ & 29.8 & 32.38 & 0.99 & & 80 & & & & & & 109 & & 22 & & 5 & & & 198 \\
\hline $4 \mathrm{H}-6$, & .3 & 33.88 & 1.036 & & 64 & & 1 & & 2 & & 4 & 5 & 106 & & 2 & & & 43 \\
\hline $4 \mathrm{H}-7$ & 32.5 & 35.08 & $\begin{array}{l}1.072 \\
1.072\end{array}$ & & 57 & 1 & & & 1 & & $\begin{array}{ll}4 & 3 \\
5 & \end{array}$ & & $10 \begin{array}{l}0 \\
15\end{array}$ & & 1 & & & 215 \\
\hline $5 \mathrm{H}$ & 3 & 36.71 & 1.122 & & 59 & 1 & 1 & & & & 63 & 3 & & & 4 & & & \\
\hline & 3 & 38.21 & 1.168 & & 42 & & 1 & & 1 & & 5 & & 8 & & & & & \\
\hline $5 \mathrm{H}-3,8$ & 36.3 & 39.71 & 1.214 & & 69 & & & & & & 1013 & & 8 & & 5 & & & 336 \\
\hline $5 \mathrm{H}-4,8$ & 37.8 & 41.21 & 1.26 & 1 & 42 & 1 & 6 & & & & 413 & & 22 & 2 & 1 & & & 2615 \\
\hline $5 \mathrm{H}-5,80-82$ & 39.3 & 42.71 & 1.305 & & 17 & & 5 & & & & 1429 & & 12 & & & & & 142 \\
\hline $5 \mathrm{H}-6$, & 40.8 & 44.21 & 1.351 & & 48 & & & & 2 & & 9 & & 4 & & 2 & & & 47 \\
\hline $5 \mathrm{H}$ & 4 & 45.41 & 1.388 & & 34 & & 4 & & & & 917 & & 3 & & & & & 75 \\
\hline & & 46.87 & 1.4 & & 7 & & & & & & 1219 & 2 & 20 & & & & & 103 \\
\hline $6 \mathrm{H}-2$ & 44.3 & 48.37 & 1.479 & & 54 & & & & 2 & & 57 & & 10 & & 3 & & & 77 \\
\hline $6 \mathrm{H}-3,80-82$ & 45.8 & 49.87 & 1.526 & & 47 & & & & 1 & & 75 & & 12 & & 1 & & & $13 \quad 3$ \\
\hline $6 \mathrm{H}-4$ & 3 & 51.37 & 1.572 & & 47 & & 5 & & & & 47 & 73 & 7 & & 1 & & & 71 \\
\hline $6 \mathrm{H}$ & & 52.87 & 1.618 & & 47 & & & & 7 & & 312 & & 7 & & 1 & & & 517 \\
\hline $6 \mathrm{~F}$ & $\begin{array}{l}40.0 \\
50.3\end{array}$ & 54.37 & 1.665 & 1 & 11 & & 7 & & 9 & & $\begin{array}{lll}5 & 12\end{array}$ & 4 & 19 & & 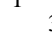 & 3 & & $\begin{array}{ll}3 & 1 \\
3 & 1\end{array}$ \\
\hline & 5 & 58 & 1.804 & 2 & 47 & & 3 & & 20 & & $\begin{array}{ll}34 \\
4\end{array}$ & & 19 & & 1 & & & 114 \\
\hline $7 \mathrm{H}-2$ & 53.8 & 60.33 & 1.851 & & 55 & & 1 & & 2 & & $\begin{array}{lll}3 & 14 \\
3\end{array}$ & $\begin{array}{ll}+4 \\
4 & 1\end{array}$ & 1 & & & & & $\begin{array}{ll}11 & 4 \\
15 & 3\end{array}$ \\
\hline $7 \mathrm{H}-3,8$ & 5 & 61.83 & 1.898 & & 5 & 1 & 4 & & 2 & & 511 & & 12 & & & & & 257 \\
\hline $7 \mathrm{H}-4$ & & 63.33 & 1.945 & & 14 & & & & 4 & & 48 & & 12 & & & & & 166 \\
\hline & & & 1.992 & & 69 & & & & 1 & & 1121 & & & & 2 & & & \\
\hline $7 \mathrm{H}$ & 59.8 & 66 & 2.0 & & 41 & 1 & & & 8 & & 45 & & 8 & & 2 & & & $\begin{array}{ll}9 & 5\end{array}$ \\
\hline & 6 & & 2.0 & & 37 & & 1 & & 1 & & $8 \quad 15$ & & & & 2 & & & 3111 \\
\hline $8 \mathrm{H}-1$, & 6 & 69.89 & 2.15 & & 47 & & & & 2 & & 45 & & 27 & & & & & 256 \\
\hline $8 \mathrm{H}-2$, & & 71.39 & 1 & & 29 & & 1 & & & & $\begin{array}{lll}6 & 14\end{array}$ & & 3 & & 1 & & & 2912 \\
\hline & & 72.91 & 2.2 & & 51 & & 4 & 1 & 1 & & 513 & & & & & & & 23 \\
\hline & & 74.37 & 2.26 & & 30 & & 1 & & & & 35 & & 17 & & & & & 84 \\
\hline $8 \mathrm{H}-$ & & 75.86 & 2.344 & & 39 & & & 1 & 2 & & $\begin{array}{lll}3 & 15\end{array}$ & & 17 & & & & & $\begin{array}{cc}0 & 4 \\
30 & 6\end{array}$ \\
\hline & 69.28 & 77.37 & 2.39 & & 67 & & & & & & $\begin{array}{ll}715 \\
\end{array}$ & & 4 & & 2 & & & $\begin{array}{ll}30 & 6\end{array}$ \\
\hline $8 \mathrm{H}-$ & & 78.59 & 2 & & 36 & & & & & & 9 & & 2 & & 2 & & & 247 \\
\hline $9 \mathrm{H}-1$ & 71 & 80 & 2.506 & 1 & 37 & & & & & & 711 & $\begin{array}{ll}1 & 2\end{array}$ & 74 & & & & & \\
\hline & & & 2.5 & 2 & 28 & & & & 9 & & 96 & & 5 & & 1 & & & $29 \quad 1$ \\
\hline & & & 2.6 & 2 & 13 & & & & 9 & & 27 & & 6 & & & & & \\
\hline $9 \mathrm{H}-$ & 75 & 85 & 2.6 & 2 & 107 & & & 1 & 3 & & 1411 & & 3 & & 1 & & & \\
\hline & & & 2.8 & 1 & 33 & 1 & & & 3 & & $\begin{array}{ll}5 & 5\end{array}$ & & 15 & & & & & $\begin{array}{lll}33 & 10\end{array}$ \\
\hline & 82 & 92 & 2 & & 5 & & 4 & & 12 & & 38 & $\begin{array}{ll}8 & 1\end{array}$ & 5 & & & & & \\
\hline & & 9 & & 2 & 36 & & & 2 & 8 & & 46 & & & & 2 & & & \\
\hline & & & & 3 & 51 & & & & 3 & & 32 & & & & & 1 & & \\
\hline & & & 3.0 & 2 & 35 & & 1 & & 3 & & 44 & $\begin{array}{ll}4 & 1\end{array}$ & 1 & & 4 & & & \\
\hline & 88.27 & 98.78 & 3.1 & 1 & 32 & & & & 5 & & 4 & & $2^{1}$ & & 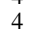 & & & \\
\hline & 89.49 & 10 & 3. & 9 & 12 & & & 1 & 4 & & 4 & & & & 2 & 2 & & $\begin{array}{ll}55 & 7\end{array}$ \\
\hline & 90 & 1 & 3 & 1 & 38 & & & & & & 10 & & 6 & & 3 & & & $23 \quad 5$ \\
\hline & & & & 2 & 47 & & & & 8 & & 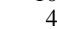 & $\begin{array}{ll}4 \quad 2 \\
4\end{array}$ & 2 & & 3 & & & \\
\hline & & & & & 54 & & & & & & 6 & 6 & 5 & & & & & 263 \\
\hline 11 & 94 & 1 & 3.3 & 2 & 20 & & 1 & & 5 & & & & 6 & & 2 & & & $27 \quad 7$ \\
\hline & 96 & 1 & 3.443 & 1 & 36 & & & & 1 & 1 & 3 & $\begin{array}{ll}3 & 1 \\
3\end{array}$ & & & 2 & & & $47 \quad 4$ \\
\hline & 97 & & & & 27 & 1 & & & & & 8 & & 1 & & 3 & & & 168 \\
\hline & & 1 & & & 47 & 1 & & & 2 & & 7 & $\begin{array}{ll}7 & 2 \\
& 2\end{array}$ & 15 & & & & & $\begin{array}{ll}41 & 11\end{array}$ \\
\hline & & & & 1 & 35 & & 1 & & 2 & & 1 & 7 & 7 & & & 1 & & $\begin{array}{l}41 \\
49\end{array}$ \\
\hline & & & & & 28 & & 6 & & 1 & & 28 & 8 & 212 & & & & & \\
\hline 12 & & 115. & 3.6 & & 17 & & 7 & & 3 & & 3 & $\begin{array}{ll}3 & 6 \\
3\end{array}$ & 312 & & 1 & & & \\
\hline & & 116.66 & 3.7 & & 8 & 2 & 2 & & & & 37 & $\begin{array}{l}7 \\
7 \\
7\end{array}$ & $\begin{array}{l}12 \\
18\end{array}$ & & & & & $\begin{array}{lll}36 & 13\end{array}$ \\
\hline & & & & & 3 & & 2 & 1 & 5 & & 10 & & 15 & & & & & 08 \\
\hline & & & & & 3 & & 14 & & 6 & & 10 & 2 & 18 & & & & & \\
\hline & & & & 1 & 11 & & 7 & & 5 & & 8 & $8 \quad 1$ & 2 & & & & & $\begin{array}{ll}34 & 8\end{array}$ \\
\hline 13 & & 1 & 3.9 & & 6 & & 6 & & 2 & & 15 & & 5 & & 1 & & & $48 \quad 6$ \\
\hline 13 & 08 & 124.45 & 4.0 & & 8 & & 3 & & & & 7 & $\begin{array}{l}7 \\
7\end{array}$ & & & 1 & $\begin{array}{l}1 \\
1 \\
1\end{array}$ & & $\begin{array}{ll}+0 \\
38 & 6 \\
38 & \end{array}$ \\
\hline 13 & 2.4 & 126.0 & $4.0 \mathrm{c}$ & & 18 & 1 & 3 & & 3 & 1 & 10 & & 1 & & 2 & & & 208 \\
\hline $13 \mathrm{H}-4,80-82$ & 113.8 & 127.45 & 4.147 & 1 & 10 & 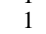 & 1 & 1 & 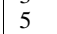 & & $4 \quad 4$ & 4 & & & & 2 & 1 & $15 \quad 5$ \\
\hline
\end{tabular}


Table 1 (continued).

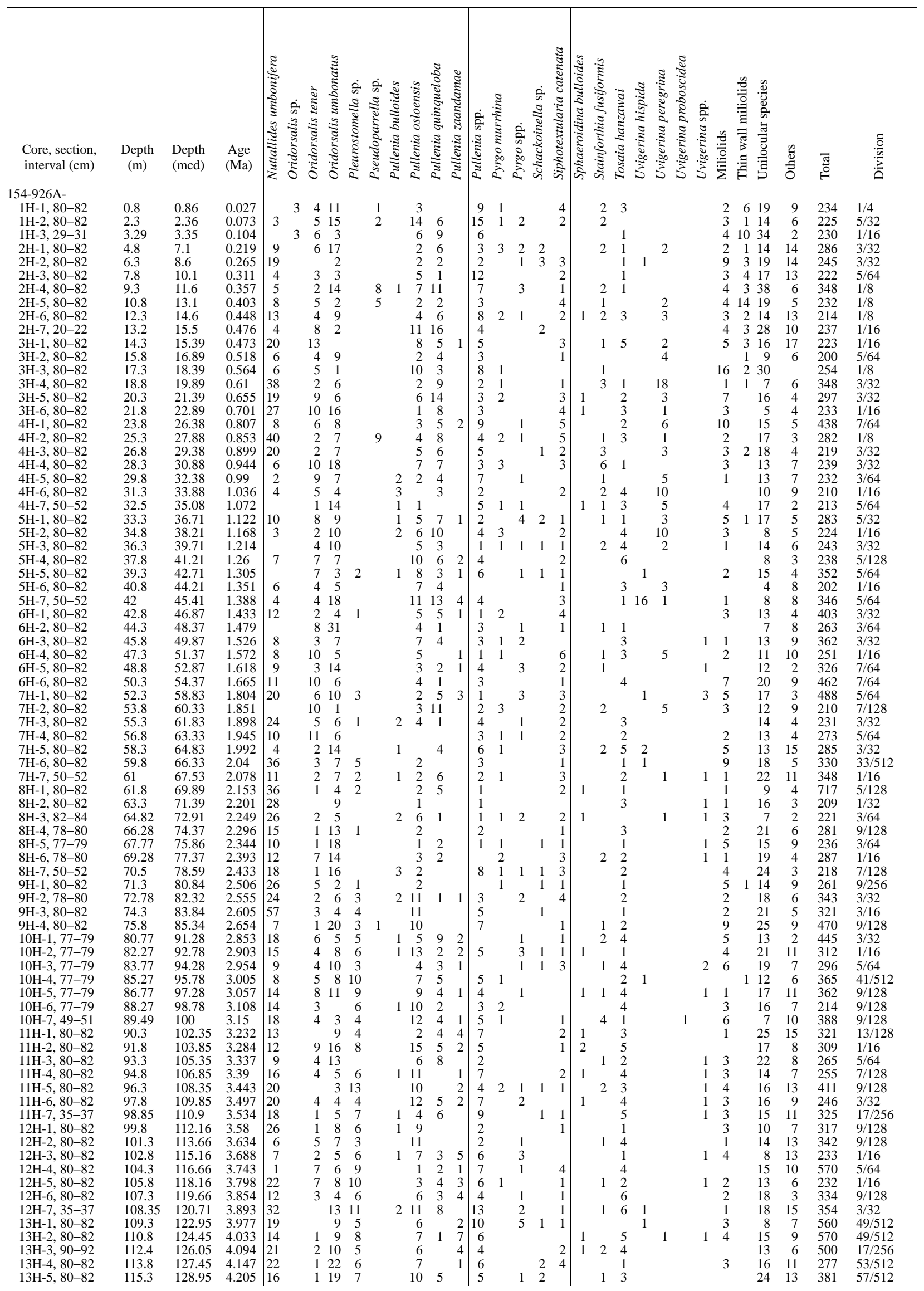


Table 1 (continued).

\begin{tabular}{|c|c|c|c|c|c|c|c|c|c|c|c|c|c|c|c|c|c|c|c|c|c|c|c|c|c|c|c|c|c|c|c|}
\hline $\begin{array}{l}\text { Core, section, } \\
\text { interval }(\mathrm{cm})\end{array}$ & $\begin{array}{l}\text { Depth } \\
\text { (m) }\end{array}$ & $\begin{array}{l}\text { Depth } \\
\text { (mcd) }\end{array}$ & $\begin{array}{l}\text { Age } \\
(\mathrm{Ma})\end{array}$ & 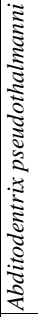 & 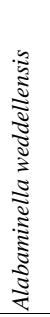 & 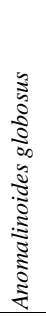 & 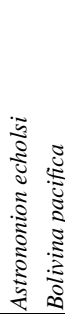 & 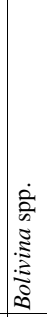 & 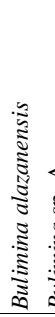 & 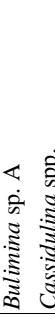 & 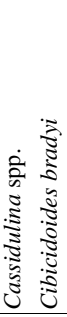 & 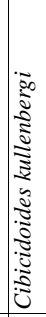 & 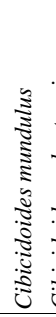 & 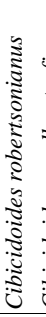 & 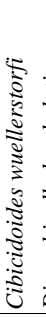 & 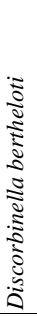 & 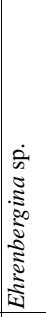 & 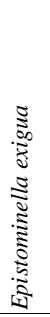 & 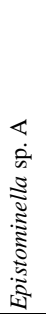 & 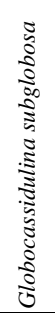 & 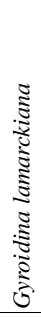 & 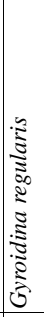 & $\begin{array}{l}0 \\
0 \\
0 \\
0 \\
0 \\
\vdots \\
0 \\
0 \\
0 \\
0\end{array}$ & 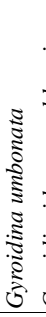 & 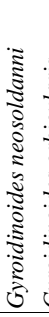 & 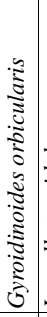 & 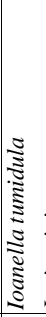 & 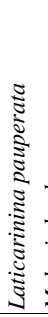 & 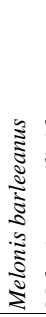 & 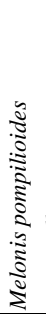 & 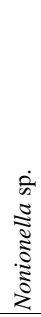 \\
\hline $13 \mathrm{H}-5,80-82$ & 15.3 & 128.95 & .205 & & 13 & 1 & & 6 & & & 93 & & & & & & & 16 & 5 & 58 & 1 & 1 & 4 & 5 & & 1 & 4 & & 1 & & 4 \\
\hline 1. & 168 & 1 & 263 & 1 & 26 & 1 & & 4 & & 1 & 9 & 1 & & & 3 & & & 40 & 4 & 30 & 3 & 3 & 1 & 2 & & 2 & 6 & & & & 2 \\
\hline $13 \mathrm{H}-7,50-52$ & 117.5 & 131.15 & 4.29 & & 26 & & 2 & 17 & & 48 & $8 \quad 3$ & 3 & & & 1 & 2 & & 31 & 3 & 25 & 4 & 3 & & 2 & 2 & 1 & 4 & & & 1 & 4 \\
\hline $14 \mathrm{H}-1,80-82$ & 118.8 & 134.61 & 4.425 & & 18 & & 2 & 15 & & & 121 & & & & & & & 22 & 5 & 44 & 1 & 2 & & 1 & 2 & & 7 & & 1 & & 1 \\
\hline $14 \mathrm{H}-2,80-82$ & 120.3 & 136.11 & 4.484 & & 24 & & 5 & 4 & 1 & 13 & 32 & 1 & & & & 1 & & 49 & 3 & 27 & 3 & 4 & & 8 & & & 2 & & & & \\
\hline $14 \mathrm{H}-3,80-82$ & 121.8 & 137.61 & 4.544 & & 12 & & 2 & 3 & & & 8 & & & & 1 & & & 19 & 7 & 23 & 9 & & & 6 & & & 9 & & & 1 & \\
\hline $14 \mathrm{H}-4,80-82$ & 123.3 & 139.11 & 4.604 & & 25 & & 1 & & & & 3 & 1 & 2 & & 1 & 4 & & 34 & 4 & 33 & 5 & & 1 & & 2 & & 5 & & & & \\
\hline $14 \mathrm{H}-5,80-82$ & 124.8 & 140.61 & 4.664 & & 12 & 2 & 2 & 11 & & & 19 & 1 & & & & 3 & & 28 & 6 & 40 & 4 & 3 & & 3 & 5 & & 7 & & 1 & & 2 \\
\hline $14 \mathrm{H}-7,80-82$ & 126.78 & 142.59 & 4.745 & & 21 & & 3 & 4 & & & 181 & & 2 & & 1 & & & 19 & 6 & 41 & 3 & 3 & 1 & 5 & 4 & & 12 & & & & 1 \\
\hline $15 \mathrm{H}-1,80-82$ & 128.3 & 145.11 & 4.848 & 1 & 15 & & 5 & 7 & & & 14 & & 11 & & & 1 & & 13 & 5 & 58 & 5 & 1 & & 7 & & & 6 & & & & 2 \\
\hline $15 \mathrm{H}-2,80-82$ & 129.8 & 146.61 & 4.91 & 1 & 25 & & 2 & 11 & 1 & & 6 & & 13 & & 1 & 3 & & 13 & 2 & 51 & 4 & 2 & & & & & 2 & & 3 & & \\
\hline $15 \mathrm{H}-3,80-82$ & 131.3 & 148.11 & 4.972 & & 23 & & & 4 & & & $\begin{array}{ll}5 & 1\end{array}$ & & 14 & & & 1 & & 39 & 2 & 44 & 3 & 6 & & & 1 & & 1 & & 4 & & 2 \\
\hline $15 \mathrm{H}-4,80-82$ & 132.8 & 149.61 & 5.035 & 1 & 27 & & 3 & & & 15 & 5 & & 10 & & & & & 41 & 2 & 32 & 8 & & & 1 & 1 & 1 & 5 & & 2 & & 1 \\
\hline $15 \mathrm{H}-5,100-10$ & 1345 & 151.31 & 5.107 & & 23 & & & 3 & & 32 & $4 \quad 1$ & & 16 & & & & & 36 & 4 & 49 & 5 & 2 & & 1 & 2 & & 4 & & 1 & 1 & \\
\hline $15 \mathrm{H}-6,80-82$ & 135.8 & 152.61 & 5.162 & & 18 & & 2 & 5 & & & 9 & & 1 & & & & & 38 & 2 & 47 & 6 & 4 & 3 & 3 & & & 4 & & & & 2 \\
\hline $15 \mathrm{H}-7,50-52$ & 136.5 & 153.31 & 5.192 & 1 & 11 & 1 & & 5 & & & 16 & 1 & 7 & & & & & 16 & 6 & 40 & 6 & 2 & 1 & 4 & 1 & & 1 & & & & 1 \\
\hline $16 \mathrm{H}-1,80-82$ & 1 & 155.76 & 5207 & 2 & 21 & & & 4 & & & $\begin{array}{ll}5 & 1\end{array}$ & & 2 & & 3 & & & 31 & 6 & 34 & 5 & 3 & & 3 & 1 & & 3 & & 1 & & \\
\hline $16 \mathrm{H}-2,90-92$ & 139.4 & 157.36 & 5.367 & 5 & 31 & & & 19 & 1 & & 14 & & 8 & & 5 & 1 & & 10 & 7 & 114 & 3 & 4 & 1 & 8 & & 2 & 5 & & 1 & & \\
\hline $16 \mathrm{H}-3,80-82$ & 140.8 & 158.76 & 5.428 & 2 & 15 & & & 9 & & 31 & 142 & & 4 & & & 1 & & 24 & 2 & 55 & 7 & & & 4 & 1 & & 5 & & & & \\
\hline $16 \mathrm{H}-4,75-77$ & 142.25 & 160.21 & 5.491 & & 14 & & & 8 & & & 14 & & 12 & & & & & 13 & 2 & 113 & 6 & 4 & & 6 & 1 & 3 & 2 & 1 & & & 1 \\
\hline $16 \mathrm{H}-5,80-82$ & 143.8 & 161.76 & 5.56 & & 10 & 1 & & 28 & & & 17 & & 15 & & 2 & & & 15 & 3 & 108 & 13 & 2 & & 11 & 1 & & 9 & & 1 & & \\
\hline $80-82$ & 145.3 & 163.26 & 5.627 & 1 & 6 & & & 27 & & & 151 & 2 & & & & & & 12 & & 82 & 5 & & 1 & 5 & 3 & & 11 & & & & \\
\hline $16 \mathrm{H}-7,50-52$ & 146.5 & 164.46 & 5.681 & 1 & 5 & & & 1 & & & 11 & & 4 & & & & & 8 & 1 & 80 & 7 & 1 & & 2 & 1 & & 7 & 1 & & & \\
\hline $17 \mathrm{H}-1,80-82$ & 1473 & 166.28 & 5.763 & & 18 & 1 & & & & & 3 & & 7 & & & & & 55 & 4 & 56 & 12 & 4 & 3 & 2 & 4 & & 7 & & 1 & & 1 \\
\hline $17 \mathrm{H}$ & 148.8 & 167.78 & 5.831 & 1 & 27 & & 1 & 1 & & & 5 & & 10 & & 1 & 1 & & 53 & 3 & 44 & 7 & 1 & 3 & 2 & & 2 & 7 & & & & \\
\hline $17 \mathrm{H}-3,80-82$ & 150.3 & 169.28 & 5.9 & 1 & 20 & & 2 & 3 & & & 9 & 2 & 1 & & & 1 & & 14 & 3 & 65 & 1 & 2 & & 1 & 1 & 2 & 10 & & 2 & & 2 \\
\hline $17 \mathrm{H}$ & 151.8 & 170.78 & 5.96 & 2 & 8 & & & 4 & 1 & & 131 & 1 & 7 & & & & & 23 & 5 & 70 & 2 & 1 & 2 & 1 & & 1 & 11 & & & & \\
\hline $17 \mathrm{H}-$ & 153.3 & 172.28 & 6.039 & 4 & 30 & 1 & 1 & 11 & & & 2 & & 4 & & 1 & & & 61 & 4 & 75 & 5 & & & 8 & 3 & 1 & 10 & & 1 & 1 & 2 \\
\hline $17 \mathrm{H}-6,80-82$ & 154.8 & 173.78 & 6.109 & 10 & 23 & 1 & 1 & 11 & & & 3 & & 12 & & & & & 28 & 5 & 47 & 6 & & & 3 & & & 3 & & & & 1 \\
\hline & 156.81 & 176.46 & 6.236 & 5 & 5 & & 1 & 8 & & & 2 & & & & & 1 & & 27 & 2 & 44 & 13 & & & 13 & 5 & & 17 & & & & 1 \\
\hline $18 \mathrm{H}-2,81-83$ & 158.31 & 177.96 & 6.308 & 1 & 25 & & 2 & 1 & & & 2 & & 10 & & & & & 15 & 3 & 61 & 13 & & & 6 & 2 & & 10 & 1 & 2 & & \\
\hline $18 \mathrm{~F}$ & 159.81 & 179.46 & 6.38 & 1 & 31 & & & & & & 7 & & 8 & 1 & 1 & & & 99 & 6 & 62 & 7 & & & 8 & 2 & & 14 & & & & \\
\hline $18 \mathrm{H}-4,81-83$ & 161.31 & 180.96 & 6.453 & & 17 & & & 3 & & & 11 & & 12 & & 1 & & & 38 & 4 & 51 & 7 & & & 3 & 2 & & 10 & & & & 1 \\
\hline $18 \mathrm{H}$ & 162.8 & 182.4 & 6.526 & & 22 & 1 & 1 & 1 & & & 11 & & & & 1 & & & 62 & 3 & 58 & 6 & 3 & & 6 & 3 & 2 & 6 & & & & 2 \\
\hline $18 \mathrm{H}-6,77-79$ & 164.27 & 183.92 & 6.598 & 3 & 45 & & & 1 & & & 4 & & 22 & & & & & 64 & 9 & 36 & 8 & 3 & 1 & 4 & & & 3 & & 2 & & 1 \\
\hline
\end{tabular}

Note: Numbers are expressed as number of specimens in the subdivided samples. Division is expressed as a fraction, which means the subdivided material to the whole sample used in this report.

anomalously low productivity is interpreted to mean that the corrosive water mass has been expanded below the depth $4000 \mathrm{~m}$. Between 3.2 and $0 \mathrm{Ma}$, the BFAR at the two deepest sites was for the most part relatively similar; at the shallower site BFAR was slightly higher, though with many exceptions.

These results may negate the depth effect of benthic foraminifer productivity or indicate that changes in not only organic matter flux, but also deep-water chemistry and circulation patterns have played a role in controlling benthic foraminifer abundances and faunal associations.

Globocassidulina subglobosa is widely distributed in the present deep ocean. Corliss (1979) reported that this species dominates the faunal assemblage where relatively warm $\left(0.6^{\circ}-0.8^{\circ} \mathrm{C}\right) \mathrm{AABW}$ is present. Boersma (1990) investigated environmental variations in the size of G. subglobosa and found a direct relationship between estimates of good carbonate preservation and higher abundances of large size G. subglobosa in the Oligocene and Miocene sections. Mackensen et al. (1995) suggested that this species is more common on steep flanks of ridges. A geographical difference, however, does not explain the historical change of G. subglobosa flux at three sites on the Ceara Rise (Figs. 9, 10).

However, the exact physicochemical factors controlling the distribution of this species are not well known. Considering the distribution patterns and depth stratification, G. subglobosa prefers the relatively fresh and less cold deep water such as NADW, or Circumpolar Deep Water. Accordingly, the difference in accumulation rates may indicate the vertical differentiation of water masses. After $3 \mathrm{Ma}$, AABW gradually intensified and the stratification of water masses began to be distinct. As a result, G. subglobosa gradually decreased in the deep sites.

Epistominella exigua and Alabaminella weddellensis are generally recognized as phytodetritus species. In this paper, two other species, Epistominella sp. A and Ioanella tumidula, are included in the phytodetritus species group. Their total accumulation rates are shown in Figures $11 \mathrm{~A}$ and $11 \mathrm{~B}$.

As these species are considered to opportunistically respond to fresh organic matter produced by phytoplankton blooming (Gooday, 1988), the change in total absolute abundances of these species closely relates to a strength of seasonality, and is an index for surface paleo-productivity. The accumulation rates of phytodetritus species have a high coefficient of correlation with those of total individuals despite the fact that phytodetritus species account for only one third of the total (Fig. 14). This suggests that the changes in paleo-productivity of total benthic foraminifers are controlled mainly by food supply.

N. umbonifera and G. subglobosa are less correlative with total individuals, especially $N$. umbonifera at the shallow site, and $G$. subglobosa at the deep site. These two species are controlled by not only food supply but also other complex ecological factors.

Although E. exigua and A. weddellensis have similar fluctuation patterns in relative abundances at every site, these species have partly different trends in accumulation rates even though both are phytodetritus species. The amplitude fluctuations of E. exigua are different at each depth site especially during the last $3.2 \mathrm{~m} . y$., and the flux of $E$. exigua is more correlative with $N$. umbonifera than $A$. weddellensis. If the productivity of E. exigua and A. weddellensis is controlled by 
Table 1 (continued).

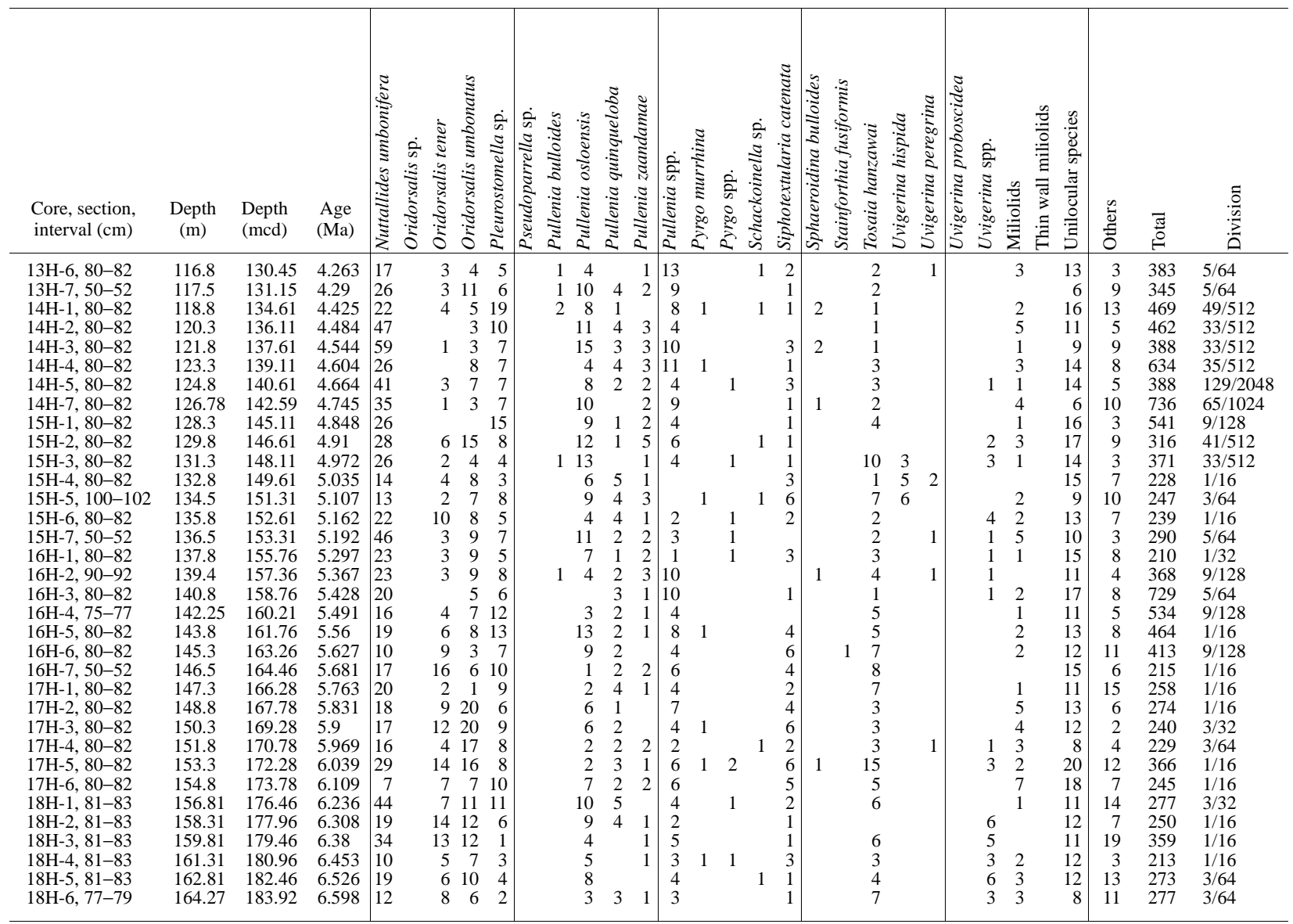

just organic matter alone, the abundance changes should not be so different at each site.

The recent depth distribution pattern of E. exigua follows the relatively high oxygen condition of the North Atlantic (Poag and Low, 1985), Indian (Peterson, 1984), and Pacific oceans (Burke, 1981). This means that this species responds to the presence of fresh deep water such as the Norwegian Over Flow Water, Antarctic Circumpolar Deep Water, and AABW. Hermelin and Shimmield (1995) reported an abundance change of this species with a 100-k.y. cyclicity in the Arabian Sea, which they interpreted as reflecting long-term changes in wind intensity, resulting in increased upwelling, and hence high surface water productivity and accumulation of phytodetritus on the seafloor. Accordingly, these facts suggest that E. exigua is influenced not only by complex ecological factors such as phytodetritus, but also by other physicochemical factors such as the oxygen content of deep water.

A. weddellensis has similar fluctuation patterns in accumulation rates at every site, with an exception prior to $4.4 \mathrm{Ma}$ at Site 928 . The similarity of fluctuations at every depth means that this species is controlled mainly by organic flux from the ocean's surface. Some other factor must account for the pattern at Site 928 before $4.4 \mathrm{Ma}$. The ecological difference of this species from E. exigua is not well known, but $A$. weddellensis is more sensitive to phytodetritus than $E$. exigua.

The accumulation rates of $N$. umbonifera began to increase at about $3 \mathrm{Ma}$ at Site 929. At the two deep sites, this species has exhibited high-amplitude fluctuations during the last 1 m.y. (Fig. 9). Living and downcore studies in benthic foraminifers have revealed that $N$. umbonifera typically associates with carbonate undersaturated bottom water (Corliss, 1979; Bremer and Lohmann, 1982), and dominates below the lysocline and above the carbonate compensation depth (Mackensen et al., 1993). The abundant occurrence of N. umbonifera directly indicates the presence of AABW (Corliss, 1979; Bremer and Lohmann, 1982; Peterson, 1984; Mackensen et al., 1995). Additionally, this species is widely distributed in the postglacial and interglacial periods (Schnitker, 1974, 1979). On the other hand, some authors consider this species as an indicator of extreme oligotrophy (Gooday, 1993; Loubere, 1994). Correlation between $N$. umbonifera and Alabaminella weddellensis fluxes, and phytodetritus species flux suggests that there is no relation or partly negative correlation of productivity between these two species (Fig. 15A) and total phytodetritus species (Fig. 15B). These results support that N. umbonifera was abundant in oligotrophy condition.

A comparison of magnetic susceptibility records with benthic foraminifer flux at Site 929 shows that the high accumulation peaks of $N$. umbonifera correlate with the lower and lowest peaks of susceptibility (Fig. 16). The fluctuation pattern of magnetic susceptibility is correlative with $\delta^{18} \mathrm{O}$ curve (Tiedemann et al.,1994), and lowest peaks of magnetic susceptibility correspond with interglacial times. A low value of magnetic susceptibility means generally high accumulation of carbonate, which is interpreted as weak carbonate dissolution or high surface productivity. As ocean paleo-productivity has been much higher in glacial than in interglacial periods (Sarnthein et al., 1988), low accumulation rates of carbonate in glacials indicate stronger dissolution than accumulation of carbonate. This suggests that this species increased during warm periods, and is an indication that the optimum environment of this species expanded in interglacial periods. This relationship is concordant with the recent distribution of this species and water mass structures (Schnitker, 1974, 1979).

As the high accumulation rates of $N$. umbonifera strongly suggest the presence of AABW (not glacial AABW) and less organic matter flux, and the BFAR at Site 929 before 3.2 Ma are similar to the lower 
Table 2. Census data for the benthic foraminifer species from Hole 928A in the 63- $\mu \mathrm{m}$ fraction.

\begin{tabular}{|c|c|c|c|c|c|c|c|c|c|c|c|c|c|c|c|c|c|}
\hline $\begin{array}{l}\text { Core, section, } \\
\text { interval }(\mathrm{cm})\end{array}$ & $\begin{array}{c}\text { Depth } \\
\text { (m) }\end{array}$ & $\begin{array}{l}\text { Depth } \\
\text { (mcd) }\end{array}$ & $\begin{array}{l}\text { Age } \\
\text { (Ma) }\end{array}$ & 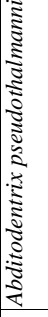 & 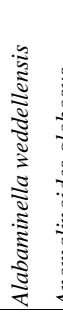 & 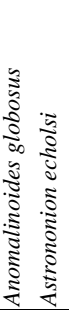 & 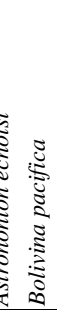 & 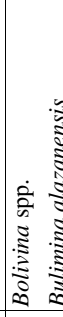 & 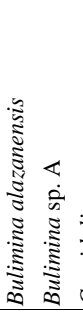 & 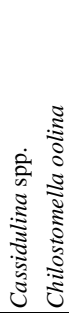 & 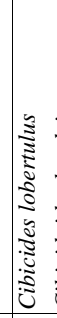 & & 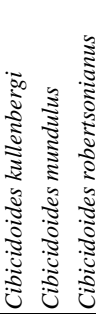 & & 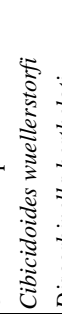 & 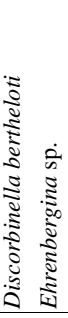 & 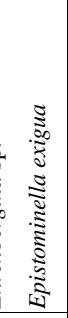 \\
\hline $\begin{array}{l}154-928 \mathrm{~A}- \\
1 \mathrm{H}-1,60-62\end{array}$ & & & & & & & & & & & & & & & & & \\
\hline $\begin{array}{l}1 \mathrm{H}-1,60-62 \\
1 \mathrm{H}-2,60-62\end{array}$ & $\begin{array}{l}0.6 \\
2.09\end{array}$ & $\begin{array}{l}0.78 \\
2.27\end{array}$ & $\begin{array}{l}0.022 \\
0.067\end{array}$ & 1 & $\begin{array}{l}52 \\
45\end{array}$ & & 11 & 1 & & 1 & & & $\begin{array}{l}6 \\
1\end{array}$ & & $\begin{array}{l}4 \\
6\end{array}$ & & $\begin{array}{l}22 \\
42\end{array}$ \\
\hline $1 \mathrm{H}-3,60-62$ & 3.58 & 3.76 & 0.111 & & 50 & & & & 7 & 1 & 2 & & $2 \quad 2$ & & & & 34 \\
\hline $1 \mathrm{H}-4,60-62$ & 5.07 & 5.25 & 0.155 & 1 & 22 & & & 2 & 2 & & & & & & 5 & & 49 \\
\hline $1 \mathrm{H}-5$, & 6.56 & 6.74 & 0.198 & & 67 & & & & 4 & & & & & & 5 & & 25 \\
\hline $1 \mathrm{H}-6,30-32$ & 7.75 & 7.93 & 0.231 & & 68 & & & & 3 & & & & 4 & & 3 & & 12 \\
\hline $2 \mathrm{H}-1,60-62$ & 9.1 & 9.48 & 0.275 & & 86 & & & & 2 & 2 & & & & & 5 & & 10 \\
\hline $2 \mathrm{H}-2,60-62$ & 10.59 & 10.97 & 0.32 & & 55 & & & & & 0 & & & 4 & & 5 & & 37 \\
\hline $2 \mathrm{H}-3,60-62$ & 12.08 & 12.46 & 0.362 & & 40 & & & 1 & 2 & 1 & & & 3 & & 3 & & 35 \\
\hline $2 \mathrm{H}-4,60-62$ & 13.57 & 13.95 & 0.411 & & 21 & & & & 2 & & & & 1 & & 1 & & 23 \\
\hline $2 \mathrm{H}-5,60-62$ & 15.06 & 15.44 & 0.455 & & 35 & & & & 2 & & & & & & & & 45 \\
\hline $2 \mathrm{H}-6,60-62$ & 16.55 & 16.93 & 0.498 & & 19 & & 2 & & & $4 \quad 1$ & & & 7 & & & & 52 \\
\hline $2 \mathrm{H}-7,30-32$ & 17.74 & 18.12 & 0.533 & & 63 & & & & & & & & 9 & 4 & $4 \quad 2$ & & 19 \\
\hline $3 \mathrm{H}-1,60-62$ & 18.6 & 20.76 & 0.604 & & 35 & & & & 2 & 4 & & & 3 & 1 & 13 & & 15 \\
\hline $3 \mathrm{H}-2,60-62$ & 20.09 & 22.25 & 0.642 & & 33 & & & & 6 & 1 & & & 9 & & 4 & & 14 \\
\hline $3 \mathrm{H}-3,60-62$ & 21.58 & 23.74 & 0.681 & & 21 & & & & 1 & 1 & & & 6 & & & & 30 \\
\hline $3 \mathrm{H}-4$, & 23.07 & 25.23 & 0.719 & & 34 & & & & & & & & 30 & & 2 & & 74 \\
\hline $3 \mathrm{H}-5,60-62$ & 24.56 & 26.72 & 0.761 & & 29 & & & & & & & & 10 & & 4 & & 19 \\
\hline $3 \mathrm{H}-6,60-62$ & 26.05 & 28.21 & 0.804 & & 44 & & 1 & 9 & & & & & 3 & & 7 & & 29 \\
\hline $3 \mathrm{H}-7,30-32$ & 27.24 & 29.4 & 0.839 & & 19 & & & & & & & & 4 & & & & 47 \\
\hline $4 \mathrm{H}-1,60-62$ & 28.1 & 30.71 & 0.878 & & 34 & & & & & & & & 1 & & 1 & & 40 \\
\hline $4 \mathrm{I}$ & 29.59 & 32.2 & 0.925 & & 53 & & & 1 & 5 & & & 3 & & & & & 4 \\
\hline $4 \mathrm{H}-3,60-62$ & 31.08 & 33.69 & 0.973 & & 65 & & 1 & & & & & & 2 & & 4 & & 27 \\
\hline $4 \mathrm{H}-4,60-62$ & 32.57 & 35.18 & 1.021 & & 57 & & & & 4 & 2 & & & 64 & & 3 & & 35 \\
\hline $4 \mathrm{H}-5,60-62$ & 34.06 & 36.67 & 1.068 & & 27 & & 2 & & & & & & 213 & & 6 & 1 & 36 \\
\hline $4 \mathrm{H}-6,60-62$ & 35.55 & 38.16 & 1.107 & & 81 & & $2 \quad 1$ & & 3 & 2 & & & 21 & & 4 & & 43 \\
\hline $4 \mathrm{H}-7,30-32$ & 36.74 & 39.35 & 1.138 & & 50 & 12 & 2 & & 5 & 1 & & 1 & 3 & & 1 & & 25 \\
\hline $5 \mathrm{H}-1,60-62$ & 37.6 & 40.62 & 1.171 & & 63 & & & & & 1 & & & 75 & & 3 & 1 & 27 \\
\hline $5 \mathrm{H}-2,60-62$ & 39.09 & 42.11 & 1.213 & & 70 & & & & & 2 & & & 27 & & 5 & & 21 \\
\hline $5 \mathrm{H}-3,60-62$ & 40.58 & 43.6 & 1.258 & & 36 & & 3 & & 2 & 3 & & & 84 & & 5 & & 31 \\
\hline $5 \mathrm{H}-4,60-62$ & 42.07 & 45.09 & 1.304 & & 20 & & 2 & 1 & 4 & & & & $4 \quad 9$ & & 4 & & 19 \\
\hline $5 \mathrm{H}-5,60-62$ & 43.56 & 46.58 & 1.351 & & 51 & & 1 & & 3 & 2 & & 3 & 415 & & & & 45 \\
\hline $5 \mathrm{H}-6,60-62$ & 45.05 & 48.07 & 1.398 & & 29 & & 1 & & 2 & 2 & & J & 7 & & 3 & & 12 \\
\hline & 46.54 & & 1.445 & & 53 & & 1 & & 5 & 1 & & & 9 & & 2 & & 19 \\
\hline $6 \mathrm{H}-1,60-61$ & 47.1 & 51.07 & 1.494 & 1 & 22 & & & & 15 & 2 & & & 69 & & 1 & & 22 \\
\hline $6 \mathrm{H}-2,60-62$ & 48.59 & 52.56 & 1.543 & & 30 & & & & 6 & 1 & & 1 & 58 & 0 & 2 & & 11 \\
\hline $6 \mathrm{H}-3,60-62$ & 50.08 & 54.05 & 1.591 & & 78 & & & 4 & 5 & & & & 12 & & 3 & & 41 \\
\hline $6 \mathrm{H}-4,60-62$ & 51.57 & 55.54 & 1.64 & & 44 & & & & 1 & & & & 10 & & 2 & & 22 \\
\hline $6 \mathrm{H}-5,60-62$ & 53.06 & 57.03 & 1.688 & & 52 & & 4 & 1 & 1 & 4 & & 1 & 2 & & 2 & & 14 \\
\hline & 54.55 & 58.52 & 1.731 & & 59 & & 1 & & & 2 & & & 13 & & 2 & & 14 \\
\hline $6 \mathrm{H}-7,30-32$ & 55.74 & 59.71 & 1.766 & & 76 & & & & 15 & 6 & & 1 & 8 & & 9 & & 8 \\
\hline $7 \mathrm{H}-1,60-62$ & 56.6 & 61.34 & 1.814 & 1 & 12 & & 2 & & 7 & 4 & & & 715 & & 9 & & 19 \\
\hline $7 \mathrm{H}-2,60-62$ & 58.09 & 62.83 & 1.858 & & 37 & & 1 & & 4 & 2 & & & 35 & & 4 & & 8 \\
\hline $7 \mathrm{H}-3,60-62$ & 59.58 & 64.32 & 1.905 & & 47 & & 1 & & & 2 & & & 16 & & & & 12 \\
\hline $7 \mathrm{H}-4,60-62$ & 61.07 & 65.81 & 1.95 & & 36 & & 1 & & 2 & 3 & & & & & 5 & & 37 \\
\hline $7 \mathrm{H}-5,60-$ & 62.56 & 67.3 & 1.995 & & 65 & & & & & 3 & & & 12 & & 1 & & 16 \\
\hline $7 \mathrm{H}-6,60-6$ & 64.05 & 68.79 & 2.039 & & 52 & & & & 1 & 4 & & & 18 & & 4 & & 27 \\
\hline $7 \mathrm{H}-7,30-32$ & 65.24 & 69.98 & 2.075 & & 52 & & 3 & 1 & 1 & 4 & & & 310 & & 2 & & 14 \\
\hline $8 \mathrm{H}-3,60-62$ & 69.08 & 74.54 & 2.212 & & 58 & & 4 & & 12 & 6 & & & 39 & & 1 & & 9 \\
\hline $8 \mathrm{H}-4,6$ & 69.79 & 75.25 & 2.234 & & 59 & & 5 & 1 & 13 & 6 & & 1 & & & 2 & & 37 \\
\hline $8 \mathrm{H}-5,60-62$ & 71.28 & 76.74 & 2.279 & & 48 & & 4 & & 1 & 5 & & 1 & & & 2 & & 104 \\
\hline & 72.77 & 78.2 & 2.325 & & 18 & & 2 & & 4 & 5 & & 1 & & & 1 & & 45 \\
\hline $8 \mathrm{H}-7,30-32$ & 73.67 & 79.13 & 2.353 & & 52 & & & & & 5 & & & 11 & & & & 86 \\
\hline $9 \mathrm{H}-1,60-62$ & 75.6 & 81.58 & 2.429 & & 81 & & & & 3 & 2 & & & 12 & & 3 & & 25 \\
\hline $9 \mathrm{H}-2,6$ & 77.09 & 83. & 2.478 & & 64 & & & & 10 & 5 & & & 15 & & & & 6 \\
\hline $9 \mathrm{H}-3,6$ & 78.5 & 84.56 & 2.533 & & 55 & & & & & 4 & & & 1 & & 2 & & 27 \\
\hline $9 \mathrm{H}-4,60-62$ & 80.07 & 86. & 2.587 & & 22 & & & & 5 & 2 & & 1 & & & 4 & & 19 \\
\hline 9 H-5, 6 & 81.5 & 87. & 2.643 & & 2 & & & & 5 & 3 & & & 1011 & & & & 39 \\
\hline $9 \mathrm{H}-6,60-62$ & 83.05 & 89. & 2.698 & 1 & 22 & & & & 213 & 1 & & & 5 & & & 2 & 36 \\
\hline $9 \mathrm{H}-7,30-3$ & 84.24 & 90. & 2.734 & & 24 & & & 4 & 6 & 4 & & & 7 & & 1 & & 76 \\
\hline $10 \mathrm{H}-1,63-65$ & 85.1 & 91. & 2.7 & & 6 & & 2 & 5 & 316 & 1 & & & & & 10 & & 18 \\
\hline $10 \mathrm{H}-2,60$ & 86.59 & 92.8 & 2.813 & & 23 & & & 5 & 2 & 1 & & & & & 1 & & 38 \\
\hline $10 \mathrm{H}-3,60-62$ & 88.08 & 94.31 & 2.859 & & 24 & & & & 2 & 2 & & 2 & & & 3 & & 45 \\
\hline $10 \mathrm{H}-4$ & 89.5 & 95. & 2.9 & & 1 & & & 1 & 4 & 2 & & & 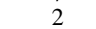 & & & & 57 \\
\hline $10 \mathrm{H}-5,61-63$ & 91.07 & 97. & 2.9 & & 32 & & & & 2 & 2 & & & 2 & & & & 55 \\
\hline & 92.5 & & & & 50 & & & & 10 & 2 & & 3 & & & 6 & & 44 \\
\hline $10 \mathrm{H}-7$ & 93. & 94 & 3. & & 32 & & & 1 & 4 & 4 & & & 9 & & 2 & & 55 \\
\hline $11 \mathrm{H}-1,60$ & 94.6 & 102.53 & 3.123 & & 38 & & & & 1 & & & & 1 & & 6 & 1 & 23 \\
\hline $11 \mathrm{H}-2,60-62$ & 96.09 & 104.02 & 3.17 & & 48 & & & & & 3 & & & 13 & & 2 & & 41 \\
\hline $11 \mathrm{H}$ & 97.58 & 105.5 & 3.216 & & 20 & 1 & & & & 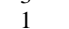 & & 1 & & & 2 & & 24 \\
\hline & 990 & 10 & 3 & & 57 & & & 1 & & 2 & & & 5 & & 2 & & 45 \\
\hline $11 \mathrm{H}-5,6 \mathrm{C}$ & 100.5 & 10 & 3. & & 42 & & 2 & & & 3 & & 2 & 12 & & & & 14 \\
\hline 11 & 10 & 10 & 3.3 & & 46 & & & & & 2 & & & 5 & & 4 & & 23 \\
\hline $11 \mathrm{H}-7,30-32$ & 103.24 & 111.17 & 3.389 & & 30 & & & & & 1 & & & 14 & & 2 & & 23 \\
\hline $12 \mathrm{H}-1,60-62$ & 104.1 & 112.52 & 3.43 & 1 & 23 & & 1 & & 3 & 2 & & 1 & 2 & & 3 & & 45 \\
\hline & 105.59 & 114.0 & 3.4 & & 23 & & & 1 & & & & 1 & & & 3 & 1 & 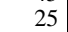 \\
\hline & & & 3 & & 26 & & & & & 7 & & & & & & & 25 \\
\hline $12 \mathrm{H}-4,60-62$ & 108.57 & 116. & 3.6 & & 25 & & & & & 1 & & & 25 & & & 1 & 24 \\
\hline $12 \mathrm{H}-5,60-62$ & 110.06 & 118.48 & 3.677 & & 13 & & 3 & & 3 & & & & 34 & & & & $1 \quad 25$ \\
\hline $12 \mathrm{H}-6,58-60$ & 111.53 & 119.95 & 3.739 & & 18 & & & & 1 & 3 & & 1 & 4 & & 2 & & 65 \\
\hline
\end{tabular}


Table 2 (continued).

\begin{tabular}{|c|c|c|c|c|c|c|c|c|c|c|c|c|c|c|c|c|c|}
\hline $\begin{array}{l}\text { Core, section, } \\
\text { interval }(\mathrm{cm})\end{array}$ & $\begin{array}{l}\text { Depth } \\
\text { (m) }\end{array}$ & $\begin{array}{l}\text { Depth } \\
\text { (mcd) }\end{array}$ & $\begin{array}{l}\text { Age } \\
\text { (Ma) }\end{array}$ & 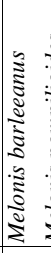 & & 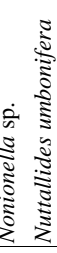 & 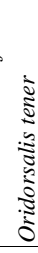 & 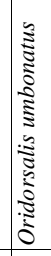 & & 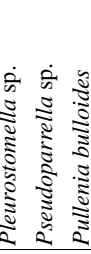 & & & & & & 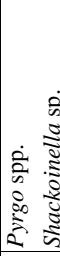 & \\
\hline $\begin{array}{l}\text { 154-928A- } \\
1 \mathrm{H}-1,60-62\end{array}$ & & & & & & & & & & & & & & & & & \\
\hline $\begin{array}{l}1 \mathrm{H}-1,60-62 \\
1 \mathrm{H}-2,60-62\end{array}$ & $\begin{array}{l}0.6 \\
2.09\end{array}$ & $\begin{array}{l}0.78 \\
2.27\end{array}$ & $\begin{array}{l}0.022 \\
0.067\end{array}$ & 5 & 1 & $\begin{array}{l}26 \\
17\end{array}$ & $\begin{array}{l}1 \\
4\end{array}$ & $\begin{array}{l}11 \\
10\end{array}$ & & & \begin{tabular}{l|l}
3 & 2 \\
1 & 8
\end{tabular} & $\begin{array}{ll}4 & 7 \\
8 & 5\end{array}$ & & $\begin{array}{l}2 \\
2\end{array}$ & 1 & $\begin{array}{lll}1 & 4\end{array}$ & 4 \\
\hline $1 \mathrm{H}-3,60-62$ & 3.58 & 3.76 & 0.111 & & & 63 & 2 & 4 & & & & 1 & 2 & 2 & 1 & & \\
\hline $1 \mathrm{H}-4,60-62$ & 5.07 & 5.25 & 0.155 & & & 269 & & 5 & & & & $7 \quad 1$ & & 1 & $\begin{array}{l}1 \\
2\end{array}$ & 14 & 4 \\
\hline $1 \mathrm{H}-5,60-62$ & 6.56 & 6.74 & 0.198 & 3 & & 46 & & 5 & & & & $\begin{array}{lll}5 & 11\end{array}$ & 1 & 1 & & & . \\
\hline $1 \mathrm{H}-6,30-32$ & 7.75 & 7.93 & 0.231 & 1 & & 324 & 3 & 19 & & & & 918 & & & 2 & 1 & s \\
\hline $2 \mathrm{H}-1,60-62$ & 9.1 & 9.48 & 0.275 & & & 211 & 6 & 11 & & & & 138 & 2 & & & & ( \\
\hline $2 \mathrm{H}-2,60-62$ & 10.59 & 10.97 & 0.32 & 3 & & 39 & 5 & 3 & & & & 95 & 3 & & 1 & & 13 \\
\hline $2 \mathrm{H}-3,60-62$ & 12.08 & 12.46 & 0.362 & & & 21 & 7 & 5 & & & & 149 & & & & & 10 \\
\hline $2 \mathrm{H}-4,60-62$ & 13.57 & 13.95 & 0.411 & 1 & 1 & 82 & 1 & 7 & & & & 34 & & 1 & & & 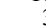 \\
\hline $2 \mathrm{H}-5,60-62$ & 15.06 & 15.44 & 0.455 & & & 63 & 3 & 2 & & & 1 & 14 & & 1 & & & 23 \\
\hline $2 \mathrm{H}-6,60-62$ & 16.55 & 16.93 & 0.498 & 1 & & 55 & 5 & 3 & & & & $\begin{array}{ll}9 & 14\end{array}$ & & & 0 & 2 & 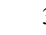 \\
\hline $2 \mathrm{H}-7,30-32$ & 17.74 & 18.12 & 0.533 & & 2 & 15 & 6 & 16 & & & 1 & 617 & 1 & 2 & & & 2 \\
\hline $3 \mathrm{H}-1,60-62$ & 18.6 & 20.76 & 0.604 & 1 & & 29 & 5 & 18 & & 0 & & 18 & & & & 2 & 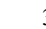 \\
\hline $3 \mathrm{H}-2,60-62$ & 20.09 & 22.25 & 0.642 & & 1 & 168 & 6 & 4 & & & & 1310 & 1 & 2 & 1 & 18 & $18 \%$ \\
\hline $3 \mathrm{H}-3,60-62$ & 21.58 & 23.74 & 0.681 & 2 & 1 & 47 & 7 & 2 & & 26 & & $\begin{array}{ll}9 & 9\end{array}$ & & 3 & & & 10 \\
\hline $3 \mathrm{H}-4,60-62$ & 23.07 & 25.23 & 0.719 & & & 99 & & 4 & & & & 68 & & 4 & & & 1 \\
\hline $3 \mathrm{H}-5,60-62$ & 24.56 & 26.72 & 0.761 & & 2 & 79 & & 8 & & & 1 & 513 & & 2 & & & 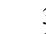 \\
\hline $3 \mathrm{H}-6,60-62$ & 26.05 & 28.21 & 0.804 & 2 & 1 & 44 & +3 & 20 & & & & 611 & & 2 & & 1 & 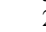 \\
\hline $3 \mathrm{H}-7,30-32$ & 27.24 & 29.4 & 0.839 & & & 93 & & 4 & & & & 2 & & 1 & & 1 & \\
\hline $4 \mathrm{H}-1,60-62$ & 28.1 & 30.71 & 0.878 & & & 67 & 6 & 9 & & & & 79 & & 2 & 1 & & 3 \\
\hline $4 \mathrm{H}-2,60-62$ & 29.59 & 32.2 & 0.925 & 4 & 1 & 31 & 7 & 8 & & & & $8 \quad 7$ & 1 & 2 & 1 & & J \\
\hline $4 \mathrm{H}-3,60-62$ & 31.08 & 33.69 & 0.973 & & 5 & 1 & 4 & 15 & & & & 105 & 1 & 2 & 1 & 2 & \\
\hline $4 \mathrm{H}-4,60-62$ & 32.57 & 35.18 & 1.021 & & 2 & 28 & & 9 & & & & 57 & & 3 & 1 & & 1 \\
\hline $4 \mathrm{H}-5,60-62$ & 34.06 & 36.67 & 1.068 & & & 330 & 7 & 13 & & & & 74 & & 2 & 1 & & 2 \\
\hline $4 \mathrm{H}-6,60-62$ & 35.55 & 38.16 & 1.107 & & & 15 & 5 & 16 & & & & 35 & & 2 & & 1 & \\
\hline $4 \mathrm{H}-7,30-32$ & 36.74 & 39.35 & 1.138 & 5 & & 1 & 7 & 5 & & 2 & & 95 & & & 1 & $\begin{array}{ll}1 & 1 \\
1 & \end{array}$ & 12 \\
\hline $5 \mathrm{H}-1,60-62$ & 37.6 & 40.62 & 1.171 & & & 11 & 4 & 12 & & & & 55 & & 7 & & & 2 \\
\hline $5 \mathrm{H}-2,60-62$ & 39.09 & 42.11 & 1.213 & & 1 & 7 & & 32 & & & & 813 & 3 & 5 & 3 & 1 & 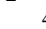 \\
\hline $5 \mathrm{H}-3,60-62$ & 40.58 & 43.6 & 1.258 & & 4 & 9 & 8 & 15 & & & & 218 & 1 & 4 & & 5 & 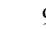 \\
\hline $5 \mathrm{H}-4,60-62$ & 42.07 & 45.09 & 1.304 & 1 & & 50 & 15 & 13 & & & & $9 \quad 1$ & 1 & 1 & 1 & & 3 \\
\hline $5 \mathrm{H}-5,60-62$ & 43.56 & 46.58 & 1.351 & 1 & 1 & 28 & 4 & 14 & & & & 106 & & 2 & 6 & & 2 \\
\hline $5 \mathrm{H}-6,60-62$ & 45.05 & 48.07 & 1.398 & & 1 & 95 & & 3 & & & & 74 & 2 & 4 & & & 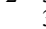 \\
\hline $5 \mathrm{H}-7,60-62$ & 46.54 & 49.56 & 1.445 & & 1 & 9 & 6 & 14 & & & & $8 \quad 5$ & & 3 & 3 & 3 & 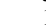 \\
\hline $6 \mathrm{H}-1,60-61$ & 47.1 & 51.07 & 1.494 & 1 & & 56 & & 5 & & & & 62 & & 1 & 1 & & 4 \\
\hline $6 \mathrm{H}-2,60-62$ & 48.59 & 52.56 & 1.543 & 1 & & 23 & 3 & 8 & & 1 & 1 & 43 & 1 & 1 & & 2 & 2 \\
\hline $6 \mathrm{H}-3,60-62$ & 50.08 & 54.05 & 1.591 & & & & 210 & 23 & & & & 88 & 2 & 5 & 2 & 1 & 1 \\
\hline $6 \mathrm{H}-4,60-62$ & 51.57 & 55.54 & 1.64 & & & 21 & & 13 & & & 2 & 34 & 1 & 1 & & & \\
\hline $6 \mathrm{H}-5,60-6$ & 53.06 & 57.03 & 1.688 & 2 & 1 & 21 & & 16 & & 1 & & 711 & 1 & 4 & & 2 & 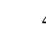 \\
\hline $6 \mathrm{H}-6,60-62$ & 54.55 & 58.52 & 1.731 & & 1 & 13 & 34 & 9 & & & & $\begin{array}{ll}5 & 14\end{array}$ & & & 1 & & 1 \\
\hline $6 \mathrm{H}-7,30-32$ & 55.74 & 59.71 & 1.766 & & & 18 & 34 & 21 & & & & 34 & & 2 & 2 & & 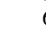 \\
\hline $7 \mathrm{H}-1,60-62$ & 56.6 & 61.34 & 1.814 & 2 & & 30 & 3 & 10 & & & & 10 & 3 & 2 & & & ser \\
\hline $7 \mathrm{H}-2,60-62$ & 58.09 & 62.83 & 1.858 & 1 & & 120 & 3 & 10 & & 4 & \begin{tabular}{l|l}
2 & 1
\end{tabular} & 114 & 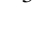 & 1 & & & 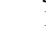 \\
\hline $7 \mathrm{H}-3,60-62$ & 59.58 & 64.32 & 1.905 & & & 25 & 53 & 11 & & & & 413 & 5 & 1 & & & \\
\hline $7 \mathrm{H}-4,60-6$ & 61.07 & 65.81 & 1.95 & 2 & & 126 & & 12 & & 4 & 1 & 2 & & & 1 & & 2 \\
\hline & 62.56 & 67.3 & 1.995 & & 1 & 6 & 54 & 8 & & 1 & & 56 & & 1 & & & 2 \\
\hline $7 \mathrm{H}-6,60-62$ & 64.05 & 68.79 & 2.039 & & & 26 & 54 & 1 & & & & 73 & & 1 & & & \\
\hline $7 \mathrm{H}-7,30-32$ & 65.24 & 69.98 & 2.075 & 2 & & 12 & 22 & 10 & & 2 & & 310 & & 1 & & & 1 \\
\hline $8 \mathrm{H}-3,60-62$ & 69.08 & 74.54 & 2.212 & 3 & & 16 & 54 & 7 & & 1 & 1 & 1 & 1 & & & & 1 \\
\hline $8 \mathrm{H}-4,60-62$ & 69.79 & 75.25 & 2.234 & 4 & & 2 & & 12 & & & & 22 & 3 & 2 & & & 1 \\
\hline $8 \mathrm{H}-5,60-62$ & 71.28 & 76.74 & 2.279 & 5 & & 41 & 1 & 9 & & 3 & & 510 & & 1 & 1 & & 1 \\
\hline & 72.77 & 78.23 & 2.325 & 2 & & 62 & 24 & 12 & & & & 2 & 2 & 1 & & & 5 \\
\hline $8 \mathrm{H}-7,30-32$ & 73.67 & 79.13 & 2.353 & 1 & & 350 & 53 & 34 & & & & 75 & & 2 & 2 & & $\begin{array}{lll}3 & 1\end{array}$ \\
\hline $9 \mathrm{H}-1,60-62$ & 75.6 & 81.58 & 2.429 & & & 25 & & 30 & & 1 & \begin{tabular}{l|l}
1 & 1 \\
\end{tabular} & 122 & & 8 & 2 & 2 & 1 \\
\hline $9 \mathrm{H}-2,60-62$ & 77.09 & 83.07 & 2.478 & 2 & & 2 & & 11 & & 1 & & 126 & 1 & 1 & & & . \\
\hline $9 \mathrm{H}-3,60-$ & 78.58 & 84.56 & 2.533 & & 2 & 21 & 5 & 20 & & & & $\begin{array}{ll}19 & 7\end{array}$ & & & & 1 & 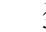 \\
\hline $9 \mathrm{H}-4,60-62$ & 80.07 & 86.05 & 2.587 & 3 & & 9 & 2 & 12 & & 4 & & 173 & 3 & & & & 1 \\
\hline & 81.58 & 87.56 & 2.643 & & & 14 & 42 & 8 & & r & & 64 & 2 & & & & 1 \\
\hline & 83.05 & 89. & 2.6 & 1 & & 24 & 42 & 8 & & 5 & & $\begin{array}{ll}8 & 7 \\
8\end{array}$ & 1 & 2 & 1 & 12 & 12 \\
\hline $9 \mathrm{H}-7,30-32$ & 84.24 & 90.22 & 2.734 & & 1 & 221 & 3 & 6 & & 1 & \begin{tabular}{l|l}
2 & 11 \\
\end{tabular} & 104 & & 2 & & & 8 \\
\hline $10 \mathrm{H}-1,6$ & 85.13 & 91 & 2.768 & & & 215 & 54 & 3 & & 3 & & 238 & 3 & 3 & 1 & & 3 \\
\hline $10 \mathrm{H}-2,6$ & 86.59 & 92.8 & 2.813 & 6 & 1 & 220 & 3 & 4 & & 1 & & 129 & & 5 & 1 & & 6 \\
\hline $10 \mathrm{H}-3,60-62$ & 88.08 & 94.31 & 2.859 & 2 & 1 & 117 & 71 & 7 & & 2 & & 145 & 1 & & & 1 & \\
\hline $10 \mathrm{H}-4,6 \mathrm{C}$ & 89.57 & 95.8 & 2.906 & 3 & & 121 & 1 & 6 & & & & $8 \quad 2$ & 2 & & & 18 & 10 \\
\hline & 91.07 & 97.3 & 2. & & & 224 & & 7 & & 3 & & 113 & & & & & \\
\hline & 0255 & 98.78 & 3. & 3 & 1 & 218 & 2 & 2 & & 6 & & 141 & 1 & 3 & & 1 & 4 \\
\hline $10 \mathrm{H}-7,30-32$ & 93.74 & 99.97 & 3. & 1 & & 29 & 2 & 7 & & 3 & & $17 \quad 3$ & 2 & 1 & & & 12 \\
\hline $11 \mathrm{H}-1,60$ & 94.6 & 102.53 & 3.123 & & & 31 & & 4 & & 2 & & 75 & & 2 & & 1 & 3 \\
\hline $11 \mathrm{H}-2,60-62$ & 96.09 & 104.02 & 3.17 & & & 316 & $5 \quad 2$ & 7 & & 5 & & 147 & 2 & 2 & & 1 & 1 \\
\hline $11 \mathrm{H}-3,60-6$ & 97.58 & 105.51 & 3.216 & 3 & & 215 & 54 & 14 & & 10 & & 125 & & 2 & 3 & 5 & \\
\hline & 99.07 & 10 & 3.2 & & 1 & 15 & & 16 & & 4 & & $17 \quad 10$ & & 7 & 4 & & \\
\hline & 10 & & & & & 12 & 2 & 10 & & 4 & & 145 & 3 & 5 & 1 & & \\
\hline $11 \mathrm{H}-6,60-$ & 102.05 & 109.98 & 3.353 & 2 & & 12 & 2 & 8 & & 4 & & 106 & 1 & 7 & & & \\
\hline $11 \mathrm{H}-7,30-32$ & 103.24 & 111.17 & 3.389 & & & 11 & 1 & 4 & & 5 & & 92 & & & 2 & & 4 \\
\hline $12 \mathrm{H}-$ & 104.1 & 112.52 & 3.43 & & & 28 & 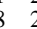 & 4 & & 3 & & 143 & & 2 & 0 & & \\
\hline $12 \mathrm{H}-2,60-6$ & 105.59 & 114.01 & 3.4 & 5 & 2 & 8 & 8 & 3 & & 6 & & 74 & & 3 & 3 & 1 & 3 \\
\hline & & & & 3 & & 13 & & 3 & & 8 & & 7 & & 5 & & 1 & 1 \\
\hline $12 \mathrm{H}-4,60$ & 108.57 & 116.9 & 3.615 & 2 & & 114 & 4 & 7 & & 3 & & 138 & & 3 & & & 1 \\
\hline $12 \mathrm{H}-5,60-62$ & 110.06 & 118.48 & 3.677 & 2 & & 23 & 3 & 3 & & 7 & & 71 & & 3 & 1 & 1 & \\
\hline $12 \mathrm{H}-6,58-60$ & 111.53 & 119.95 & 3.739 & & & 3 & 3 & 3 & & 4 & & 121 & & 2 & 5 & & \\
\hline
\end{tabular}


Table 2 (continued).

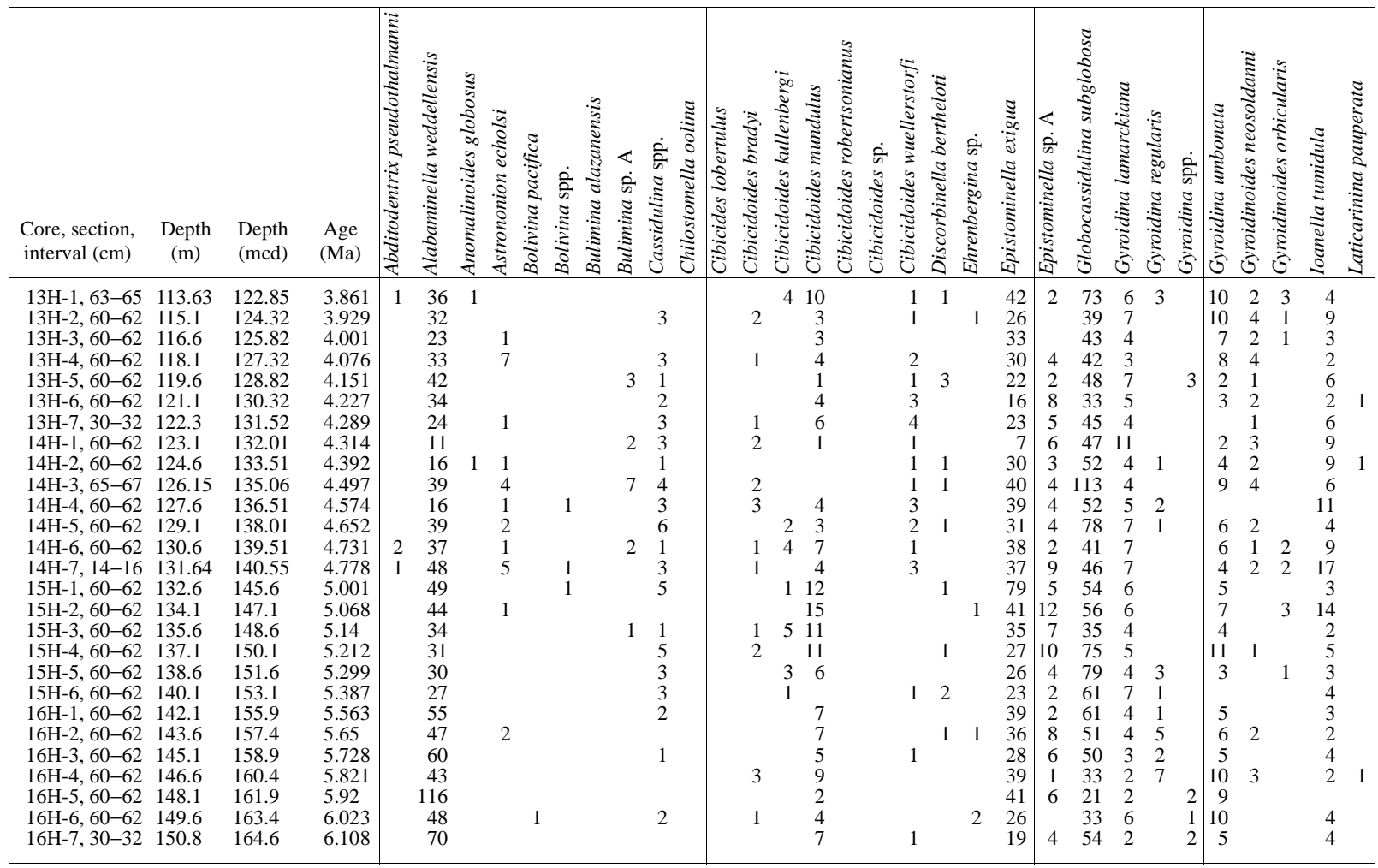

Note: Numbers are expressed as number of specimens in the subdivided in the subdivided samples. Division is expressed as a fraction, which means the subdivided material to the whole sample used in this report.

or lowest values during the last 3 m.y., the conditions of the water mass before $3.2 \mathrm{Ma}$ at $4300 \mathrm{~m}$ depth is considered to be basically the same as glacial conditions after $3 \mathrm{Ma}$. It is noted that in the oxygen isotope results, the average ice volume before $3.2 \mathrm{Ma}$ is the same as that of interglacial periods since the initiation of northern hemisphere glaciation (Tiedemann et al., 1994). However, accumulation rates of total individuals at Sites 926 and 928, with the exception of the last 0.8 m.y. at Site 928 , indicate that paleo-productivity had not changed so much during the last 6 m.y.

It has been observed for the last glacial that NADW formation decreased relative to formation of AABW (Oppo and Fairbanks, 1987; Boyle and Keigwin, 1982, 1987), and the bottom ocean was occupied by an Antarctic source of water. Benthic foraminiferal faunas, especially $N$. umbonifera abundance, indicate that the characteristics of modern bottom water (AABW) is different from that of glacial southern source bottom water. Before 3.2 Ma, the deepest site (Site 929) is clearly influenced by corrosive bottom water. In other words, the present type of AABW circulation had not existed in or had not reached the deep equatorial Atlantic between 6 and 3.2 Ma. After 3.2 $\mathrm{Ma}$, the deep-water circulation and bottom-water production, especially during interglacial times, were enhanced by the increased amplitude of glacial-interglacial contrast.

It is evident that $N$. umbonifera evolved to exploit the bottom-water environment of AABW and, expanding its niche, kept in step with the enlargement of bottom-water production in the Antarctic area.

\section{SUMMARY}

The ODP Leg 154 depth transect indicates drastic changes in deep-water environments during the past 6 m.y.

1. At 3.2 Ma, benthic foraminiferal accumulation rates below $4000 \mathrm{~m}$ changed drastically. Prior to $3.2 \mathrm{Ma}$, the accumulation rates of both total specimens and Nuttallides umbonifera are low, which indicates that the bottom-water mass was different from the present.

2. Prior to $3.2 \mathrm{Ma}$, deep-water circulation patterns, especially AABW, are similar to those of glacial periods after $3 \mathrm{Ma}$.

3. High relative abundances and accumulation rates of $N$. umbonifera are correlative with low peaks in the susceptibility records. This suggests that this species was widely distributed in the interglacial periods.

4. Phytodetritus species abundance changes indicate the same fluctuation pattern as total benthic foraminiferal accumulation rates.

\section{ACKNOWLEDGMENTS}

I would like to thank all scientists from the shipboard party of ODP Leg 154, and the crew and technical staff on the JOIDES Resolution for their scientific support. I express my appreciation to Dr. Detmar Schnitker for useful discussions. I thank Dr. Paul Pearson for critically reading the manuscript, and an anonymous reviewer for comments and suggestions that improved the paper. Discussions with Dr. Ellen Thomas about phytodetritus species and faunal changes was very helpful. I also thank Miss Maniko Kamei, Kumi Mieno, and Mr. Kanda for assistance in sample preparation. This research was supported by the Japanese Science Foundation (No. 05640517).

\section{REFERENCES}

Altenbach, A.V., and Sarnthein, M., 1989. Productivity record in benthic Foraminifers. In Berger, W.H., Smetacek, V.S., and Wefer G. (Eds.), Productivity of the Oceans: Present and Past: New York (Springer-Verlag), 255-269.

Berger, W.H., and Diester-Haass, L., 1988. Paleoproductivity: the benthic planktonic ratio in foraminifera as a productivity index. Mar. Geol., $81: 15-25$. 
Table 2 (continued).

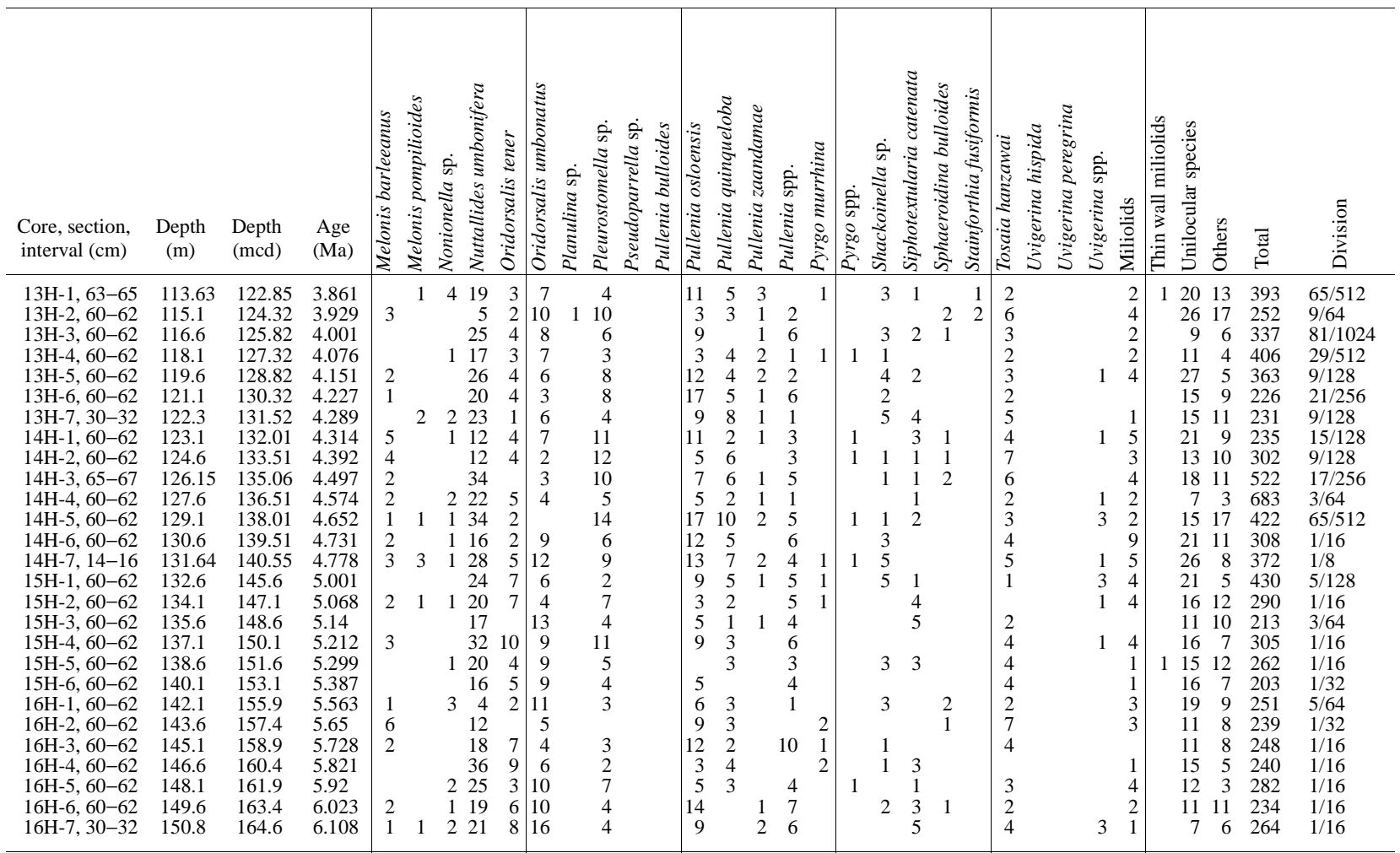

Boersma, A., 1990. Late Oligocene to late Pliocene benthic foraminifers from depth traverses in the Central Indian Ocean. In Duncan, R.A., Backman, J., Peterson, L.C., et al., Proc. ODP, Sci. Results, 115: College Station, TX (Ocean Drilling Program), 315-379.

Boyle, E.A., and Keigwin, L.D., 1982. Deep circulation of the North Atlantic over the last 200,000 years: geochemical evidence. Science, 218:784787.

1987. North Atlantic thermohaline circulation during the past 20,000 years linked to high-latitude surface temperature. Nature, 330:35-40.

Bremer, M.L., and Lohmann, G.P., 1982. Evidence for primary control of the distribution of certain Atlantic Ocean benthonic foraminifera by degree of carbonate saturation. Deep-Sea Res. Part A, 29:987-988.

Burke, S.C., 1981. Recent benthic foraminifera of the Ontong Java plateau. J. Foraminiferal Res., 11:1-19.

Campbell, J.W., and Aarup, T., 1992. New production in the North Atlantic derived from seasonal patterns of surface chlorophyll. Deep-Sea Res. Part A, 39:1669-1694.

Corliss, B.H., 1979. Recent deep-sea benthonic foraminiferal distributions in the southeast Indian Ocean: inferred bottom-water routes and ecological implications. Mar. Geol., 31:115-138.

$\longrightarrow$ 1983. Distribution of Holocene deep-sea benthonic foraminifera in the southwest Indian Ocean. Deep-Sea Res. Part A, 30:95-117.

Corliss, B.H., and Honjo, S., 1981. Dissolution of deep-sea benthonic foraminifera. Micropaleontology, 27:356-378.

Curry, W.B., Shackleton, N.J., Richter, C., et al., 1995. Proc. ODP, Init. Repts. 154: College Station, TX (Ocean Drilling Program).

Gooday, A.J., 1988. A response by benthic foraminifera to the deposition of phytodetritus in the deep sea. Nature, 332:70-73.

, 1993. Deep-sea benthic foraminiferal species which exploit phytodetritus: characteristic features and controls on distribution. Mar. Micropaleontol., 22:187-205.

Gooday, A.J., and Lambshead, P.J.D., 1989. Influence of seasonally deposited phytodetritus on benthic foraminiferal populations in the bathyal Atlantic: the species response. Mar. Ecol. Prog. Ser., 58:53-67.

Herguera, J.C., 1992. Deep-sea benthic foraminifers and biogenic opal: glacial to postglacial productivity changes in the western equatorial Pacific. Mar. Micropaleontol., 19:79-98.
Herguera, J.C., and Berger, W.H., 1991. Paleoproductivity: glacial to postglacial change in the western equatorial Pacific, from benthic foraminifera. Geology, 19:1173-1176.

Hermelin, J.O.R., 1991. The benthic foraminiferal faunas of Sites 725, 726, and 728 (Oman Margin, northwestern Arabian Sea). In Prell, W.L., Niitsuma, N., et al., Proc. ODP, Sci. Results, 117: College Station, TX (Ocean Drilling Program), 55-87.

Hermelin, J.O.R., and Shimmield, G.B., 1990. The importance of the oxygen minimum zone and sediment geochemistry on the distribution of Recent benthic foraminifera from the NW Indian Ocean. Mar. Geol., 91:1-29.

- 1995. Impact of productivity events on the benthic foraminiferal fauna in the Arabian Sea over the last 150,000 years. Paleoceanography, 10:85-116.

Loeblich, A.R., Jr., and Tappan, H., 1988. Foraminiferal Genera and Their Classification: New York (Van Nostrand Reinhold).

, 1994. Foraminifers of the Sahul shelf and Timor Sea. Spec. Publ. Cushman Found. Foraminiferal Res., 31:1-661.

Lohmann, G.P., 1978. Abyssal benthonic foraminifera as hydrographic indicators in the western South Atlantic Ocean. J. Foraminiferal Res., 8:634.

Loubere, P., 1994. Quantitative estimation of surface ocean productivity and bottom water oxygen concentration using benthic foraminifers. Paleoceanography, 9:723-737.

Lutze, G.F., and Coulbourn, W., 1984. Recent benthic foraminifera from the continental margin of Northwest Africa: community structure and distribution. Mar. Micropaleontol., 8:361-401.

Mackensen, A., Fütterer, D.K., Grobe, H., and Schmiedl, G., 1993. Benthic foraminiferal assemblages from the eastern South Atlantic Polar Front region between $35^{\circ}$ and $57^{\circ} \mathrm{S}$ : distribution, ecology and fossilization potential. Mar. Micropaleontol., 22:33-69.

Mackensen, A., Schmiedl, G., Harloff, J., and Giese, M., 1995. Deep-sea foraminifera in the South Atlantic Ocean: ecology and assemblage generation. Micropaleontology, 41:342-358.

Mackensen, A., Sejrup, H., and Jansen, E., 1985. The distribution of living and dead benthic foraminifera on the continental slope and rise off southwest Norway. Mar. Micropaleontol., 9:275-306.

Mead, G.A., 1985. Recent benthic foraminifera in the Polar Front region of the southwest Atlantic. Micropaleontology, 31:221-248. 
Miao, Q., and Thunell, R.C., 1993. Recent deep-sea benthic foraminiferal distributions in the South China and Sulu Seas. Mar. Micropaleontol., 22:1-32.

Murray, J., 1973. Distribution and Ecology of Recent Foraminifera: Baltimore, MD (Johns Hopkins Press).

Oppo, D.W., and Fairbanks, R.G., 1987. Variability in the deep and intermediate water circulation of the Atlantic Ocean during the past 25,000 years: Northern Hemisphere modulation of the Southern Ocean. Earth Planet. Sci. Lett., 86:1-15.

Peterson, L.C., 1984. Recent abyssal benthic foraminiferal biofacies of the eastern equatorial Indian Ocean. Mar. Micropaleontol., 8:479-519.

Peterson, L.C., and Lohmann, G.P., 1982. Major change in Atlantic deep and bottom waters 700,000 yr ago: benthonic foraminiferal evidence from the south Atlantic. Quat. Res., 17:26-38.

Poag, C.W., and Low, D., 1985. Environmental trends among Neogene benthic foraminifers at Deep Sea Drilling Project Site 548, Irish continental margin. In de Graciansky, P.C., Poag, C.W., et al., Init. Repts. DSDP, 80 (Pt. 1): Washington (U.S. Govt. Printing Office), 489-503.

Rathburn, A.E., and Corliss, B.H., 1994. The ecology of living (stained) deep-sea benthic foraminifera from the Sulu Sea. Paleoceanography, 9:87-150

Sarnthein, M., Winn, K., Duplessy, J.-C., and Fontugne, M.R., 1988. Global variations of surface ocean productivity in low and mid latitudes: influence on $\mathrm{CO}_{2}$ reservoirs of the deep ocean and atmosphere during the last 21,000 years. Paleoceanography, 3:361-399.

Schnitker, D., 1974. West Atlantic abyssal circulation during the past 120,000 years. Nature, 248:385-387.

-, 1979. Cenozoic deep water benthic foraminifera, Bay of Biscay. In Montadert, L., Roberts, D.G., et al., Init. Repts. DSDP, 48: Washington (U.S. Govt. Printing Office), 377-413.

, 1980. Quaternary deep sea benthic foraminifers and bottom water masses. Annu. Rev. Earth Planet. Sci., 8:343-370.
1993. Deep-sea benthic foraminifers: food and bottom water masses. In Zahn, R., Pedersen, T.F., Kaminski, M.A., and Labeyrie, L. (Eds.), Carbon Cycling in the Glacial Ocean: Constraints on the Ocean's Role in Global Change: Berlin (Springer-Verlag), NATO ASI Ser. 1, 17:539-554.

Smart, C.W., King, S.C., Gooday, A.J., Murray, J.W., and Thomas, E., 1994. A benthic foraminiferal proxy of pulsed organic matter paleofluxes. Mar. Micropaleontol., 23:89-100.

Streeter, S.S., 1973. Bottom water and benthonic foraminifera in the North Atlantic: glacial-interglacial contrasts. Quat. Res., 3:131-141.

Streeter, S.S., and Shackleton, N.J., 1979. Paleocirculation of the deep North Atlantic: 150,000 year record of benthic foraminifera and oxygen-18. Science, 203:168-171.

Thomas, E., 1992. Cenozoic deep-sea circulation: evidence from deep-sea benthic foraminifera. In Kennett, J.P., and Warnke, D. (Eds.), The Antarctic Paleoenvironment: A Perspective on Global Change. Am. Geophys. Union, Antarct. Res. Ser., 56:141-165.

Thomas, E., Booth, L., Maslin, M., and Shackleton, N.J., 1995. Northeastern Atlantic benthic foraminifera during the last 45,000 years: changes in productivity seen from the bottom up. Paleoceanography, 10:545-562.

Thomas, E., and Gooday, A.J., 1996. Cenozoic deep-sea benthic foraminifera: tracers for changes in oceanic productivity? Geology, 24:355-358.

Tiedemann, R., Sarnthein, M., and Shackleton, N.J., 1994. Astronomic timescale for the Pliocene Atlantic $\delta^{18} \mathrm{O}$ and dust flux records of Ocean Drilling Program Site 659. Paleoceanography, 9:619-638.

Woodruff, F., and Savin, S.M., 1989. Miocene deepwater oceanography. Paleoceanography, 4:87-140.

Date of initial receipt: 2 January 1996

Date of acceptance: 31 May 1996

Ms 154SR-107 
Table 3. Census data for the benthic foraminifer species from Holes 929A and 929B in the 63- $\mu \mathrm{m}$ fraction.

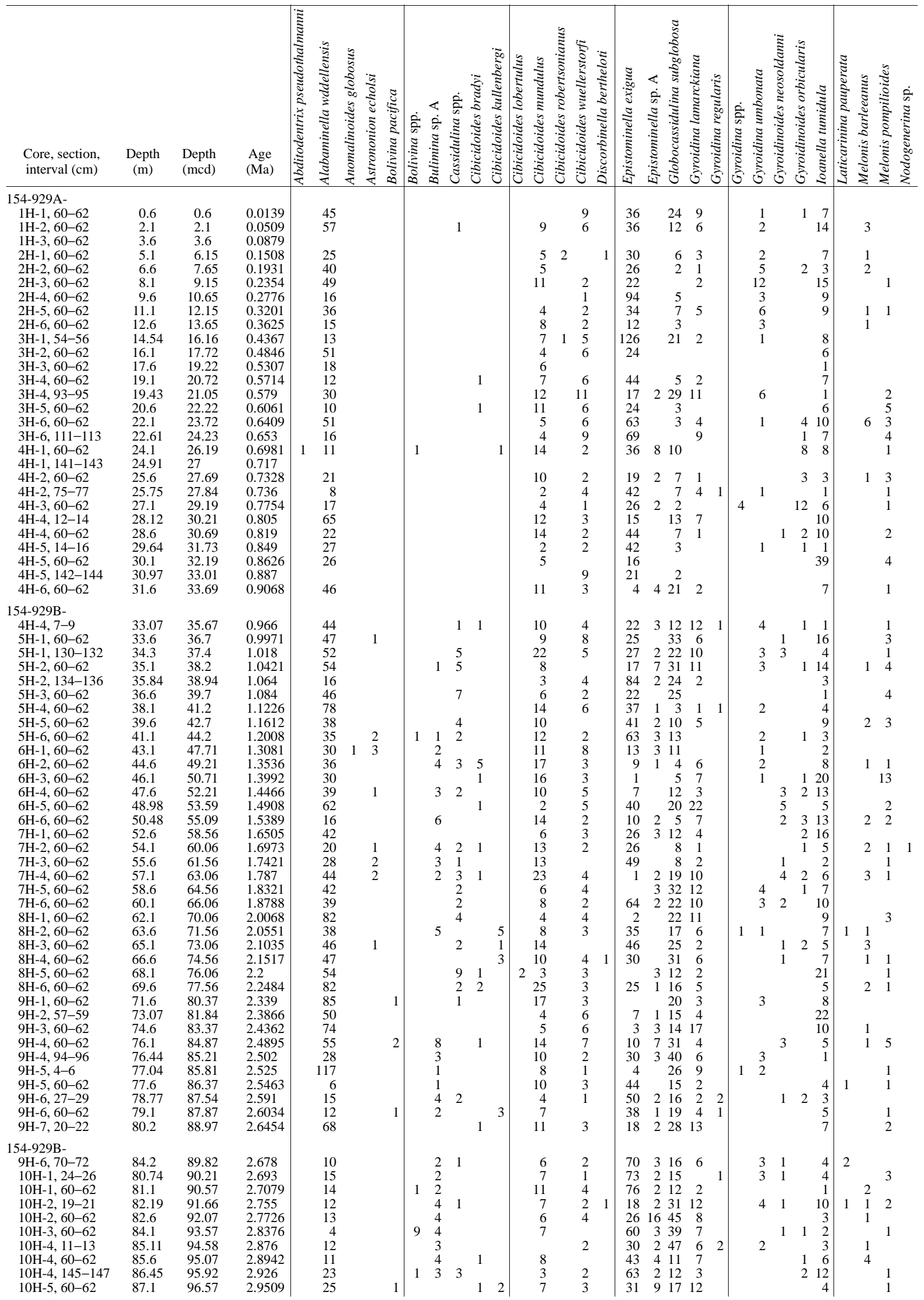


Table 3 (continued).

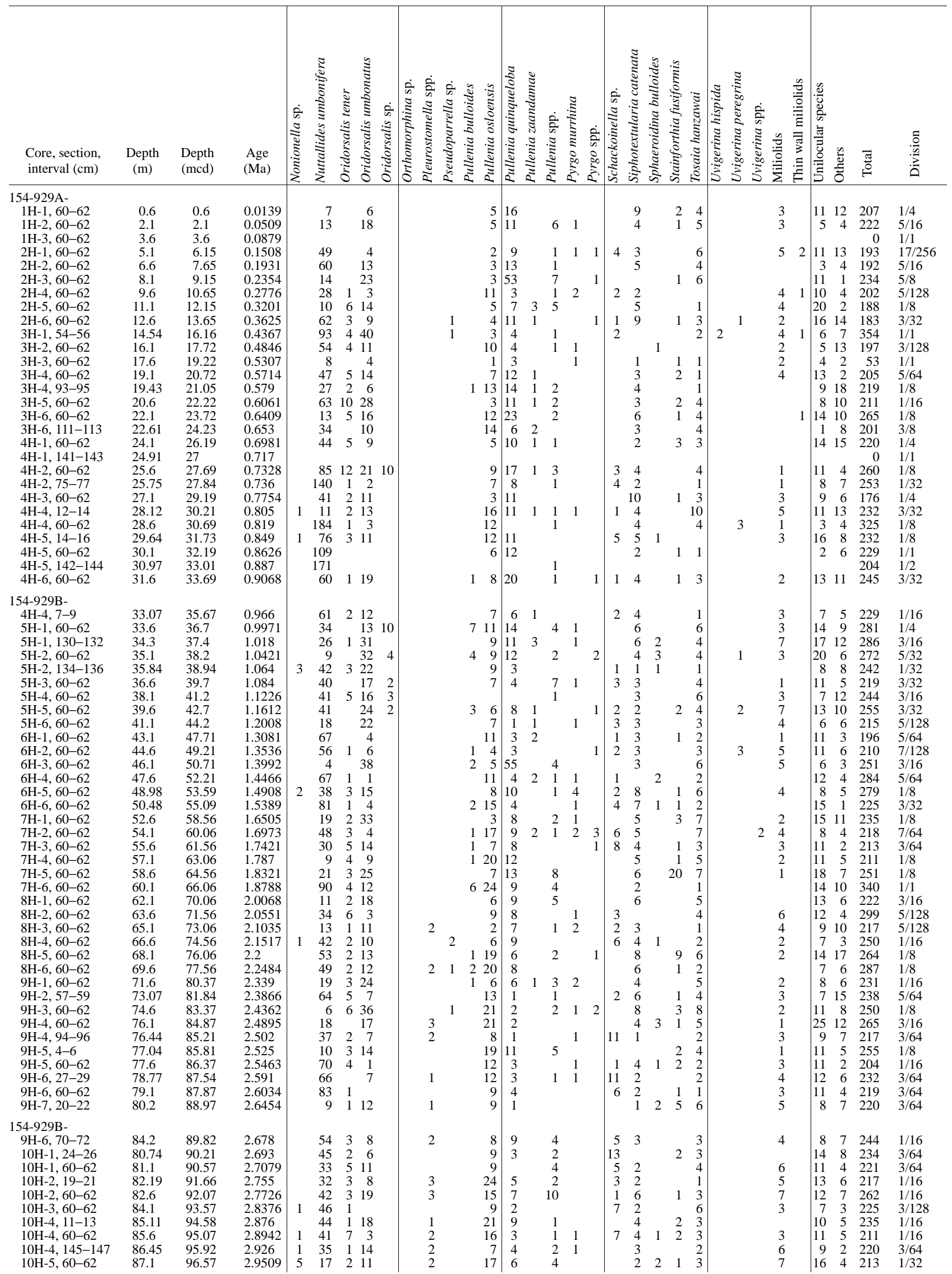


Table 3 (continued).

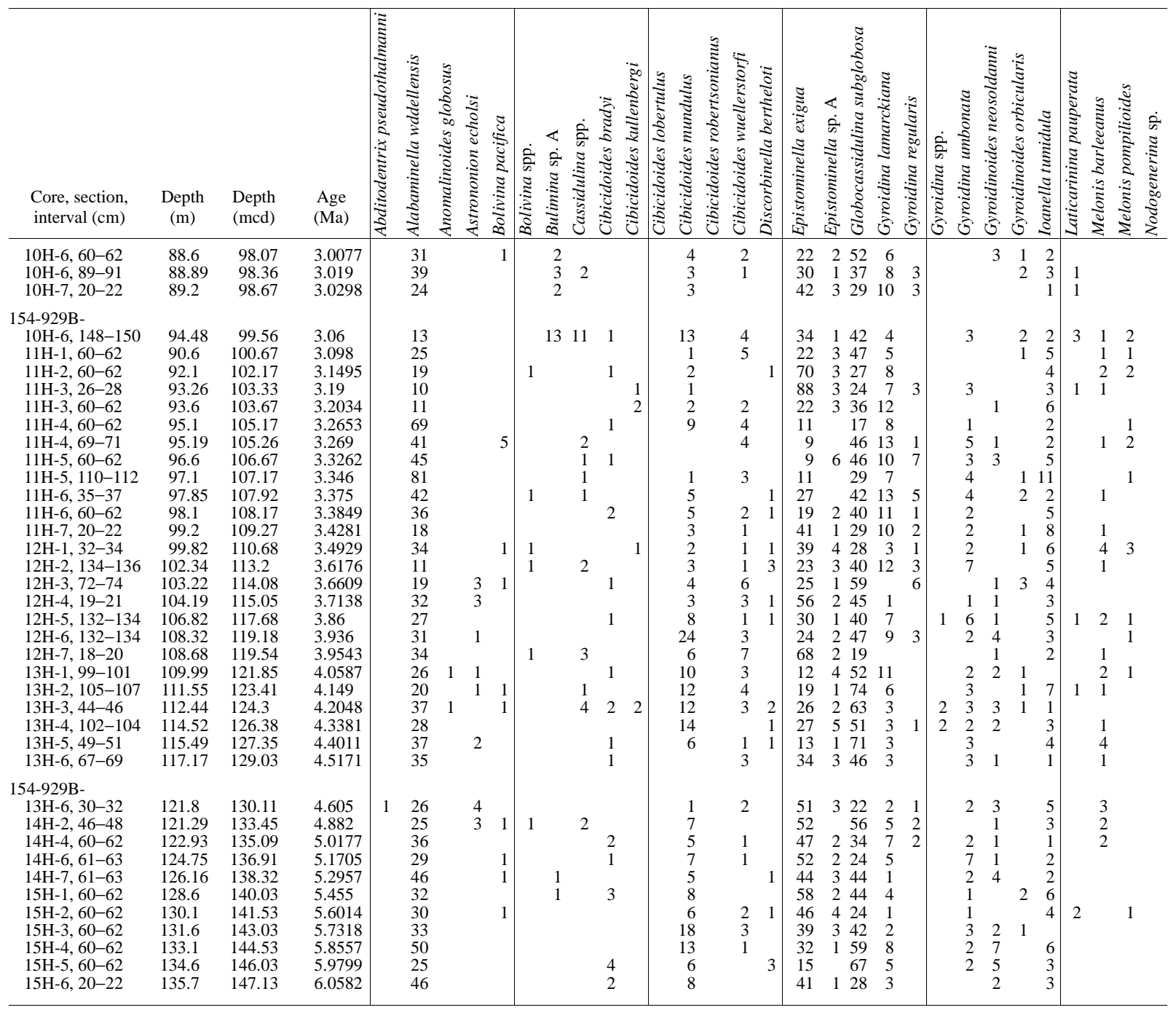

Notes: Numbers are expressed as number of specimens in the subdivided samples. Division is expressed as a fraction, which means the subdivided material to the whole sample used in this report. 
Table 3 (continued).

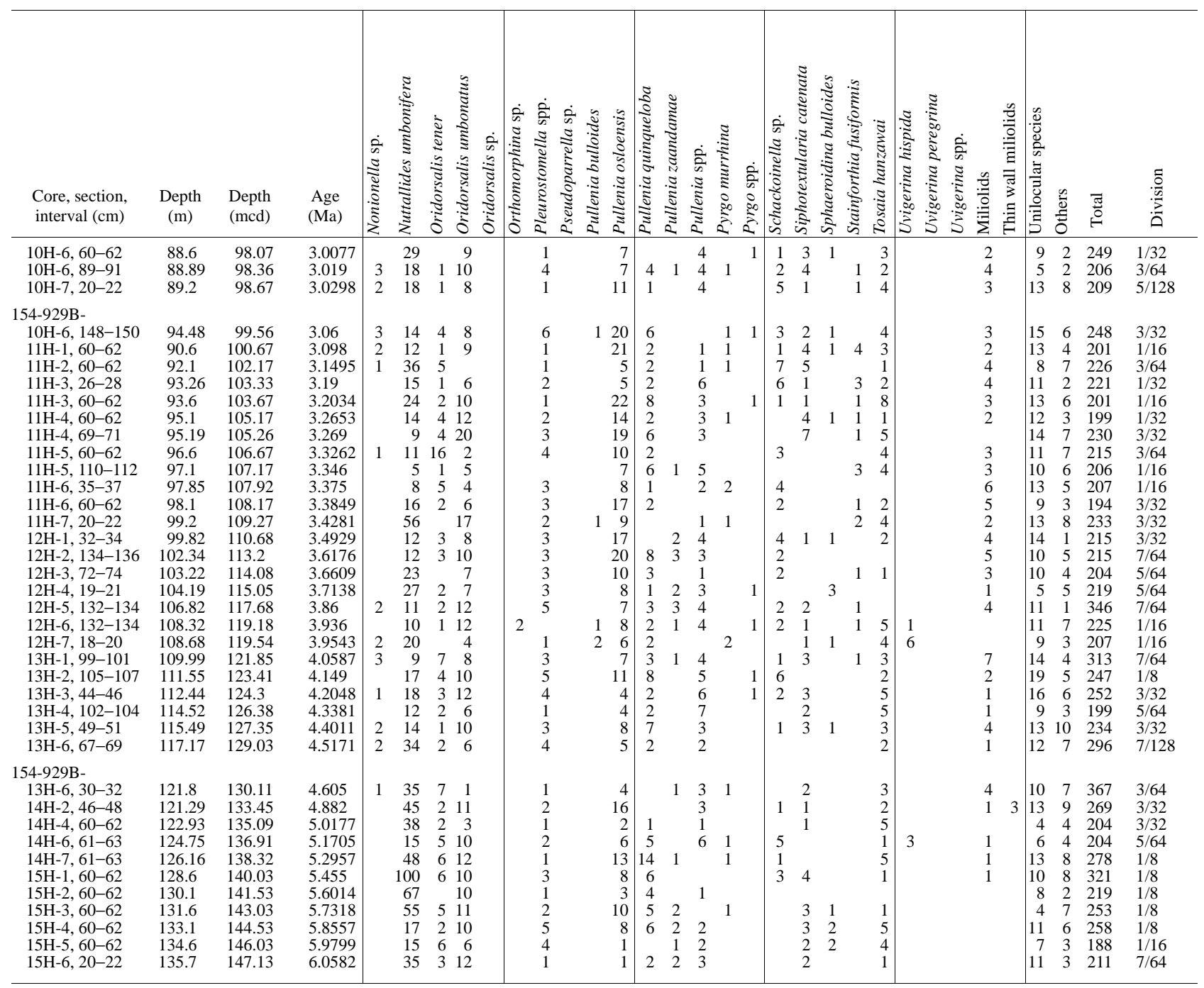



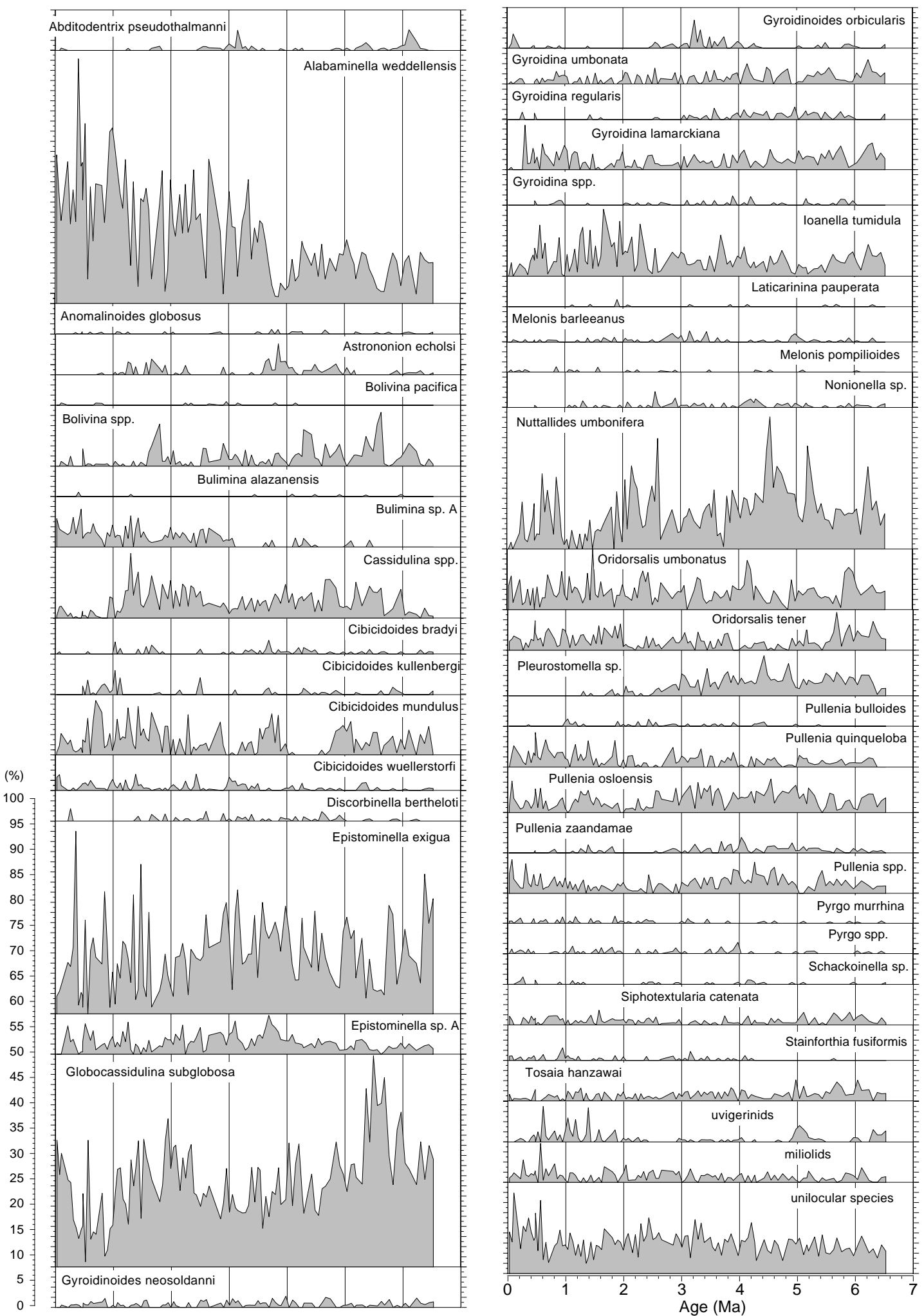

Figure 2. Relative abundances of selected benthic foraminiferal species in Hole 926A. 

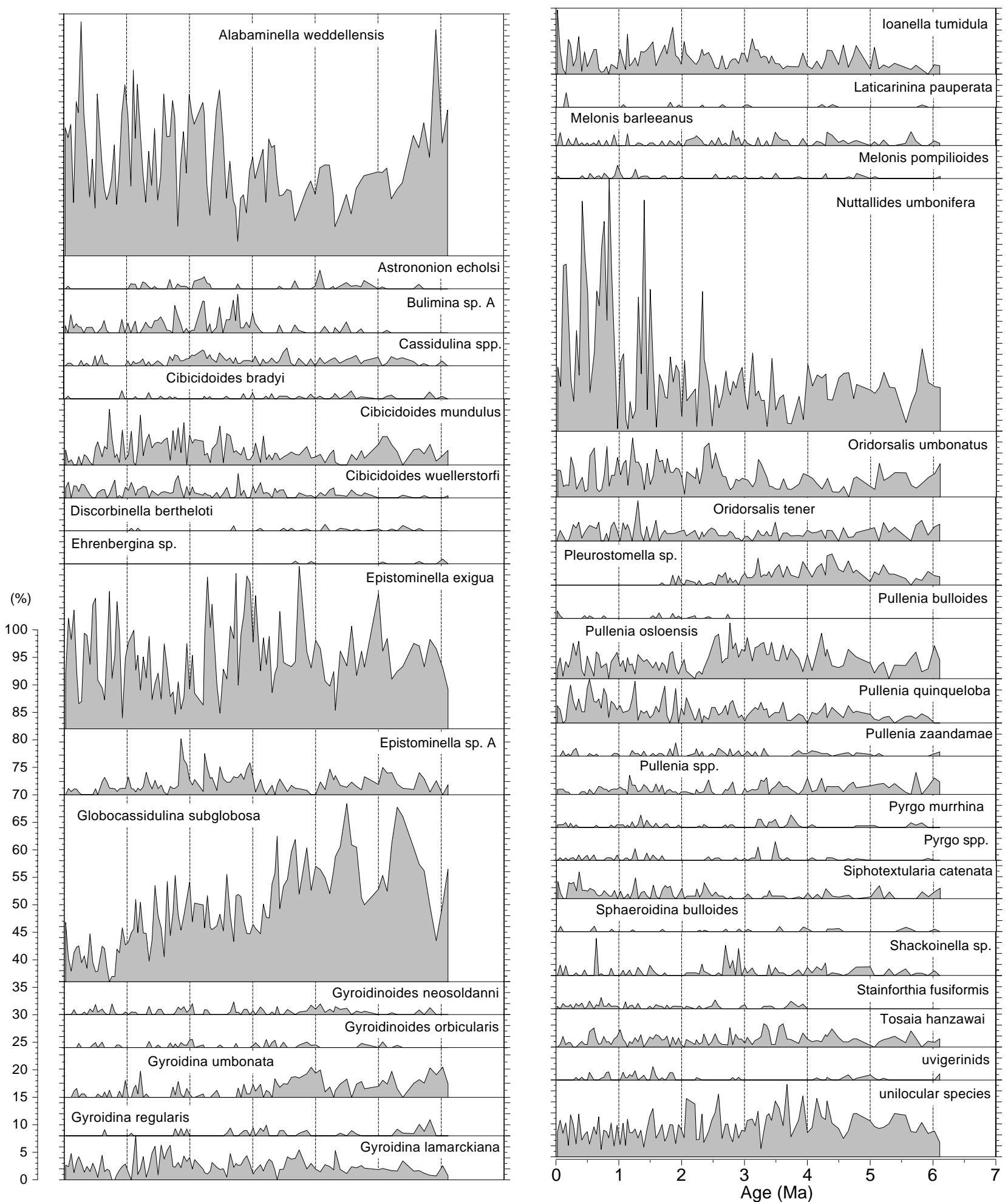

Figure 3. Relative abundances of selected benthic foraminiferal species in Hole 928A. 

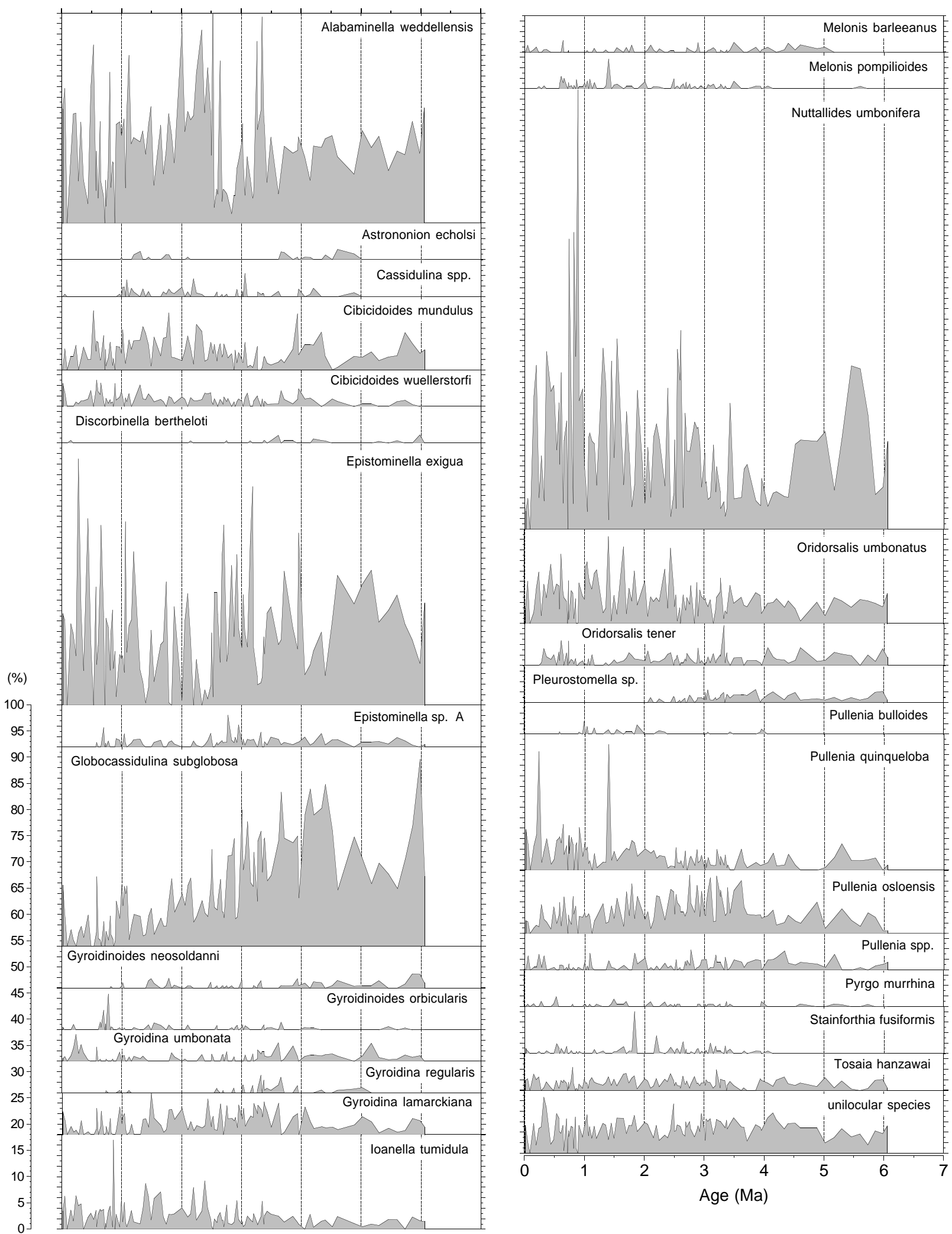

Figure 4. Relative abundances of selected benthic foraminiferal species in Hole 929A. 

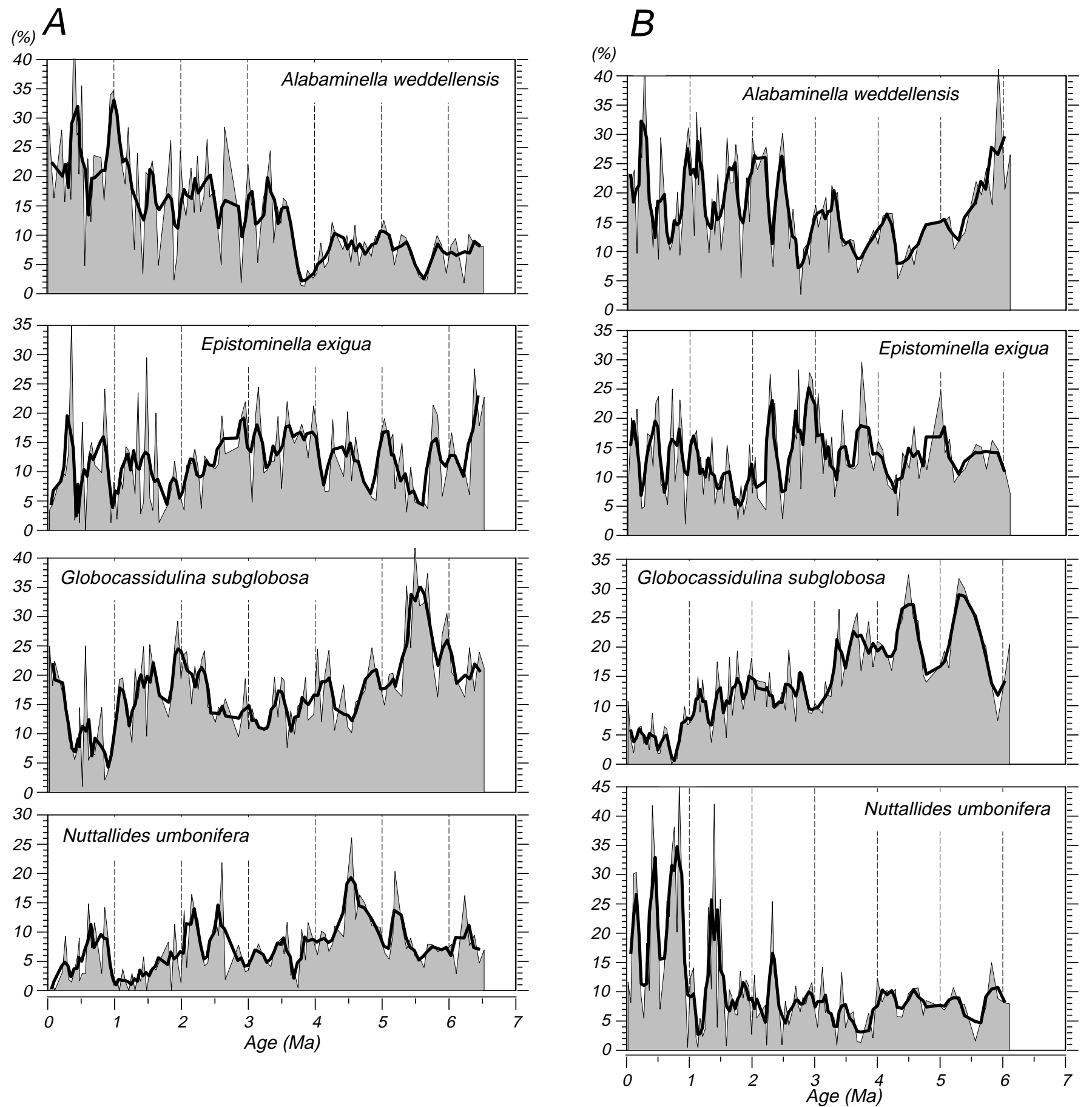

Figure 5. Relative abundance area graphs for four abundant species with superimposed curves of three-point moving averages for Holes (A) 926 A, (B) 928 A, and $(\mathbf{C})$ 929A. 

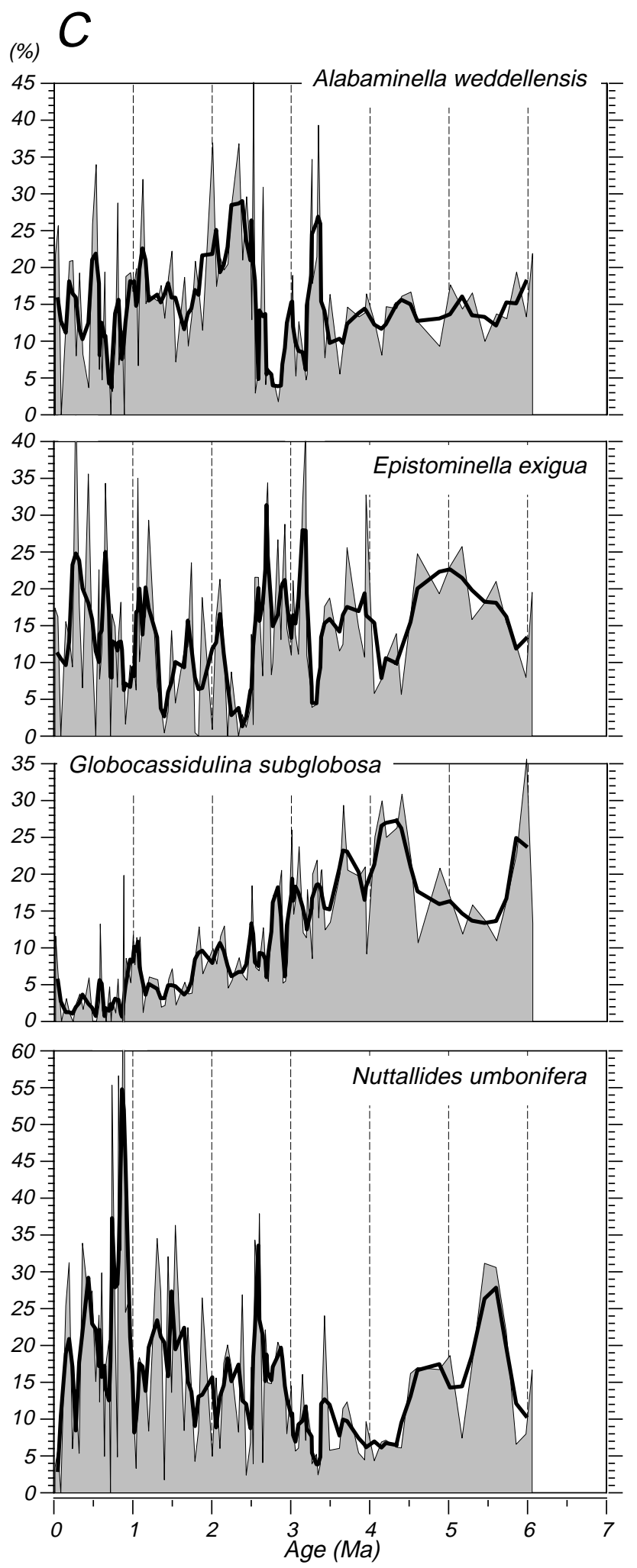

Figure 5 (continued). 

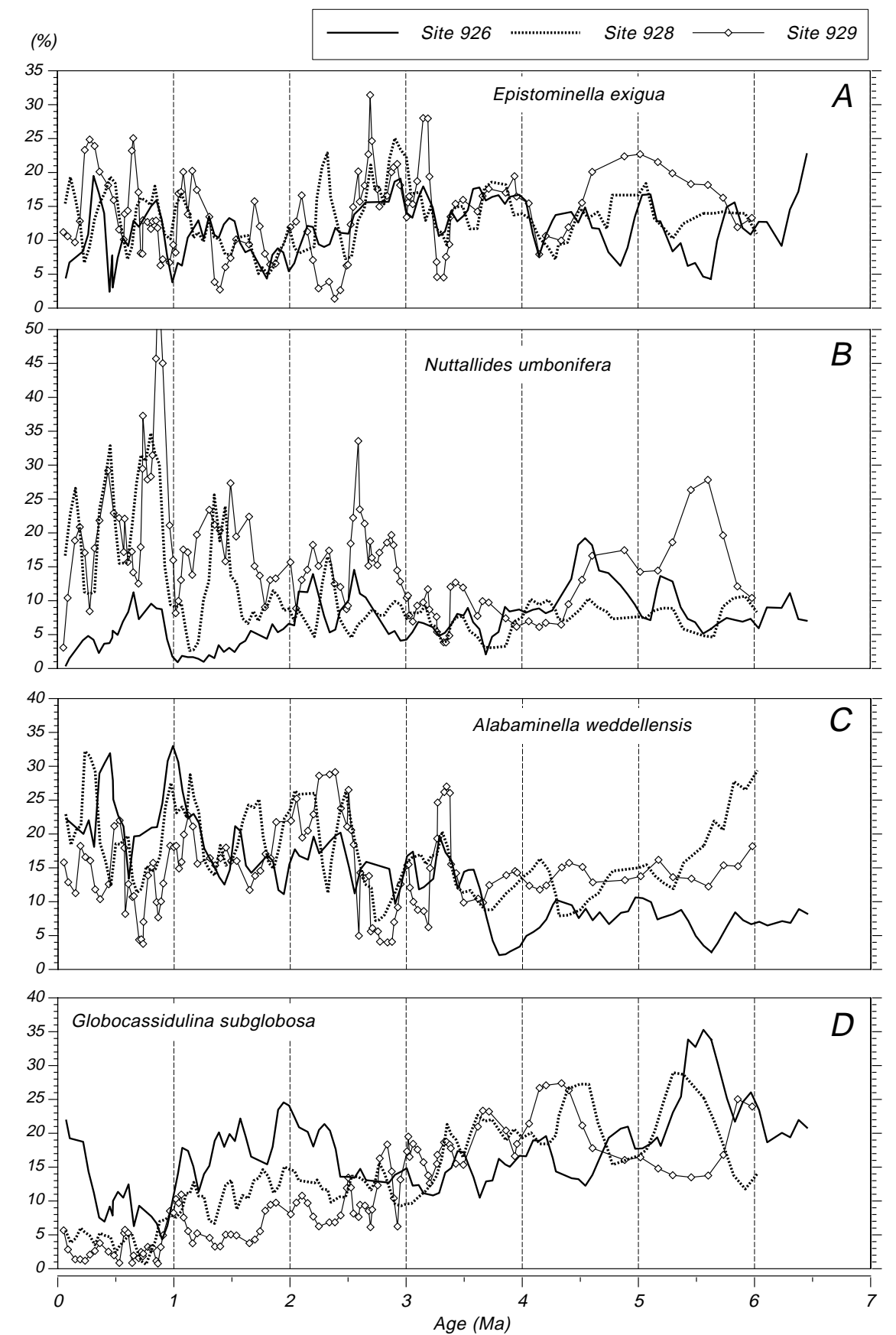

Figure 6. Three-point moving average curves of relative abundances for four species in Holes 926A, 928A, and 929A. A. Epistominella exigua. B. Nuttallides umbonifera. C. Alabaminella weddellensis. D. Globocassidulina subglobosa. 
(\%)
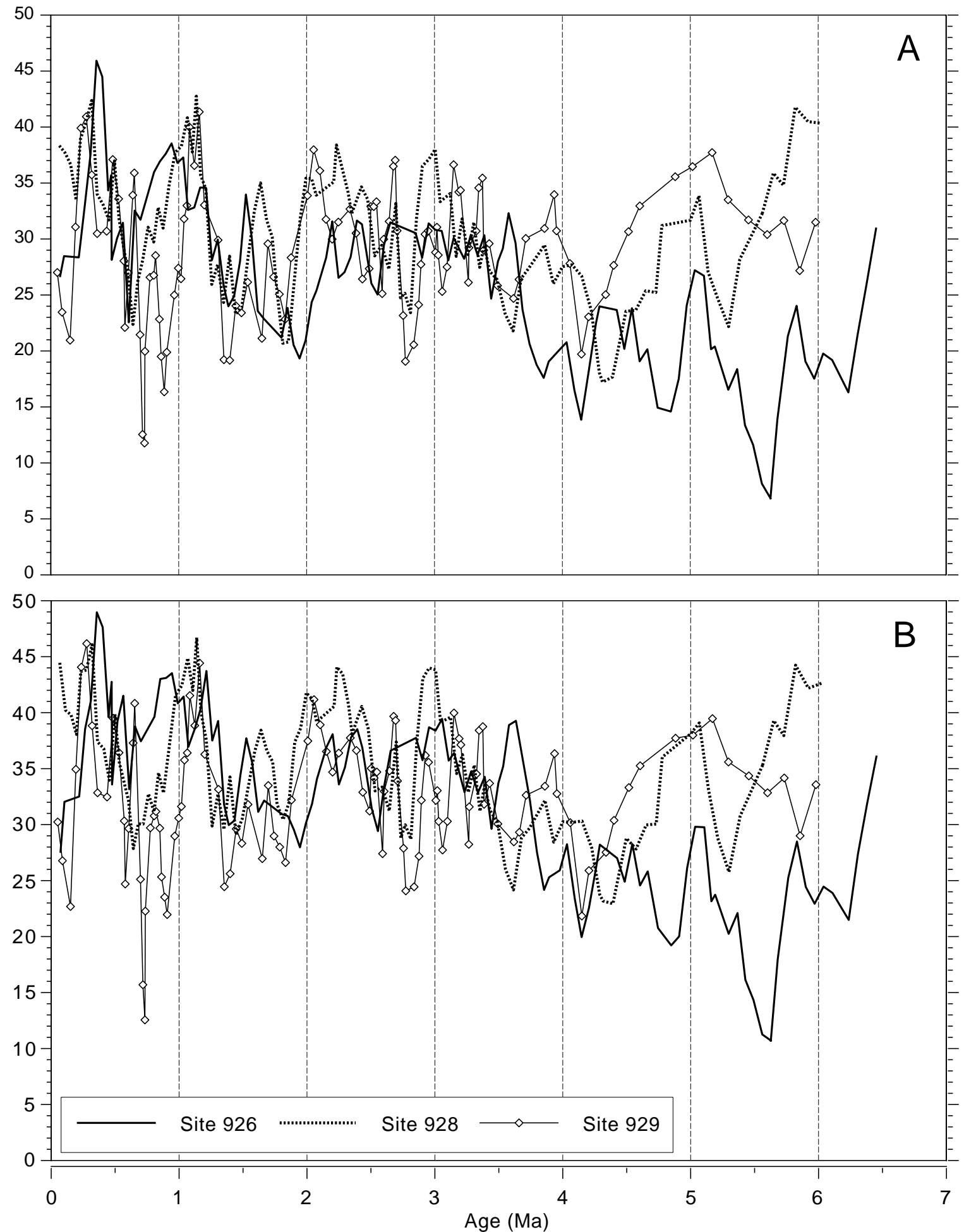

Figure 7. Relative abundance curves for phytodetritus species. A. Epistominella exigua and Alabaminella weddellensis. B. Epistominella sp. A and Ioanella tumidula are added to above two species. 
A

(\%)
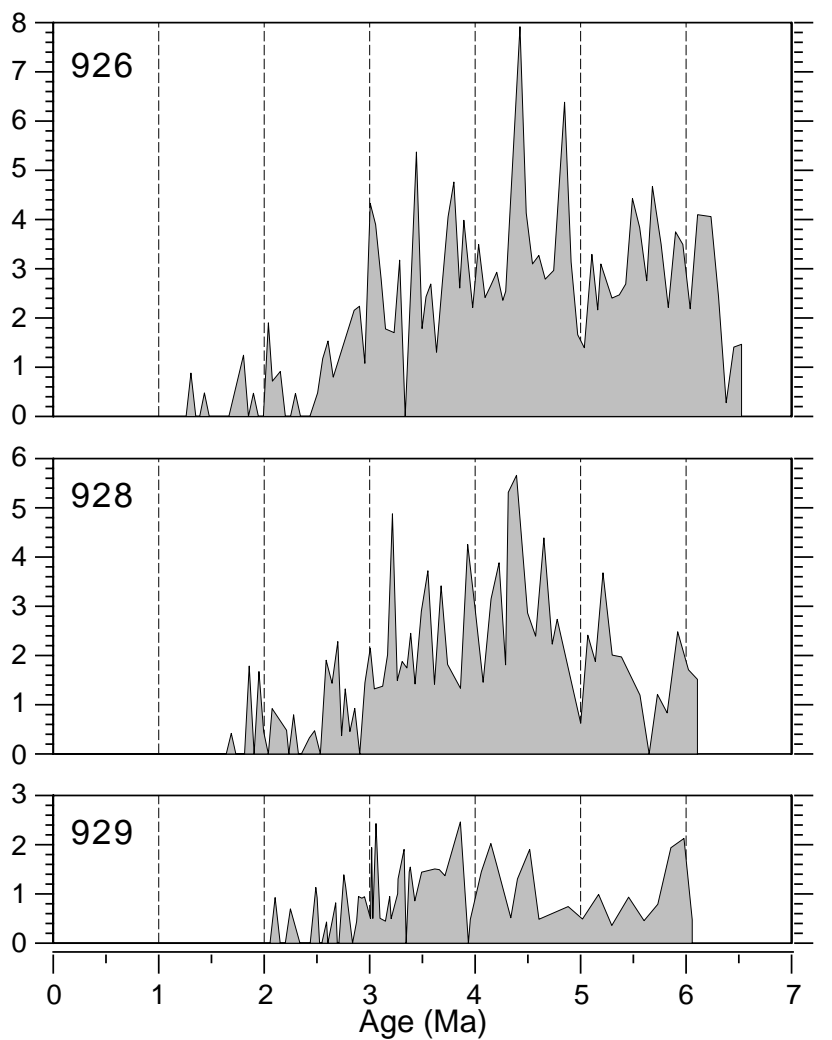

B

(\%)
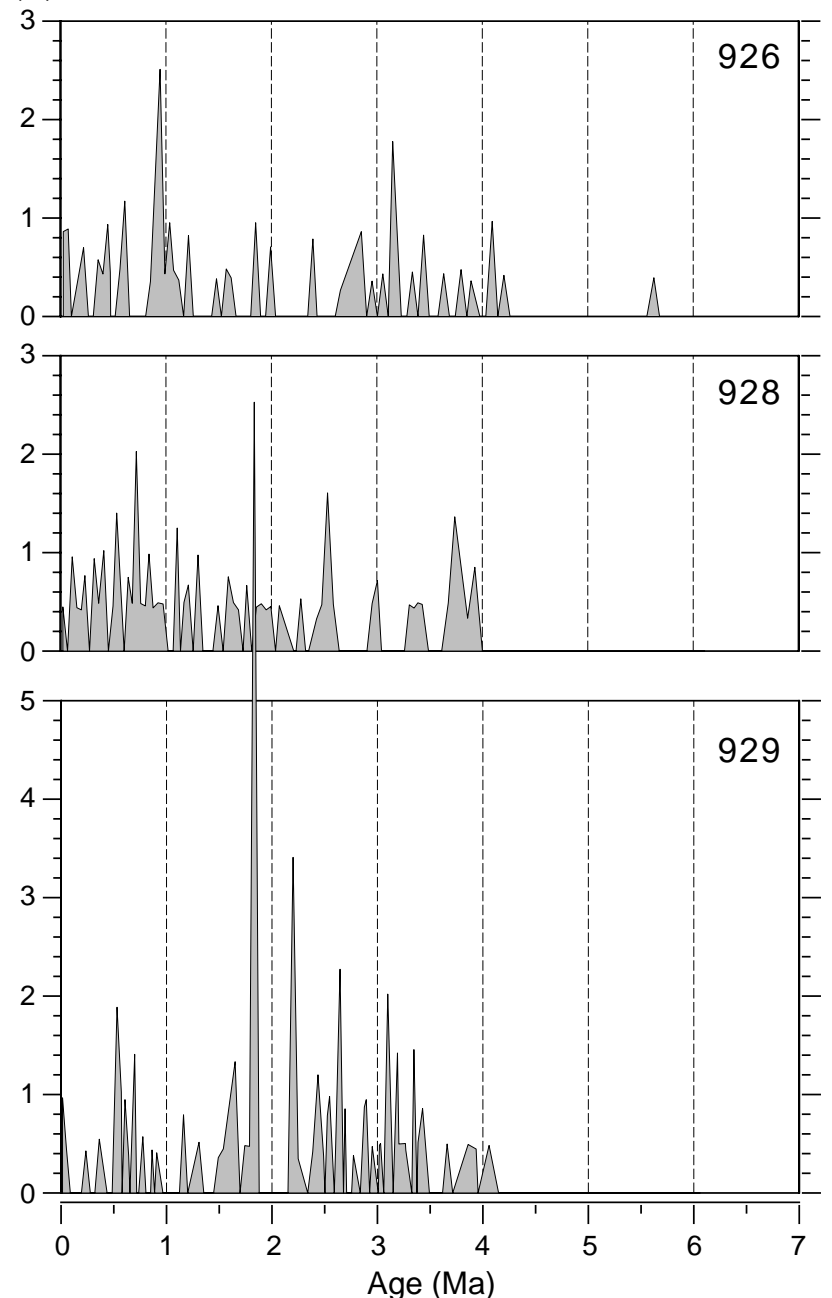

Figure 8. Relative abundance graphs for selected benthic foraminiferal species, which have stratigraphically characteristic occurrences in Holes 926A, 928A, and 929A. A. Pleurostomella spp. B. Stainforthia fusiformis. C. Pullenia osloensis. D. Ioanella tumidula. 


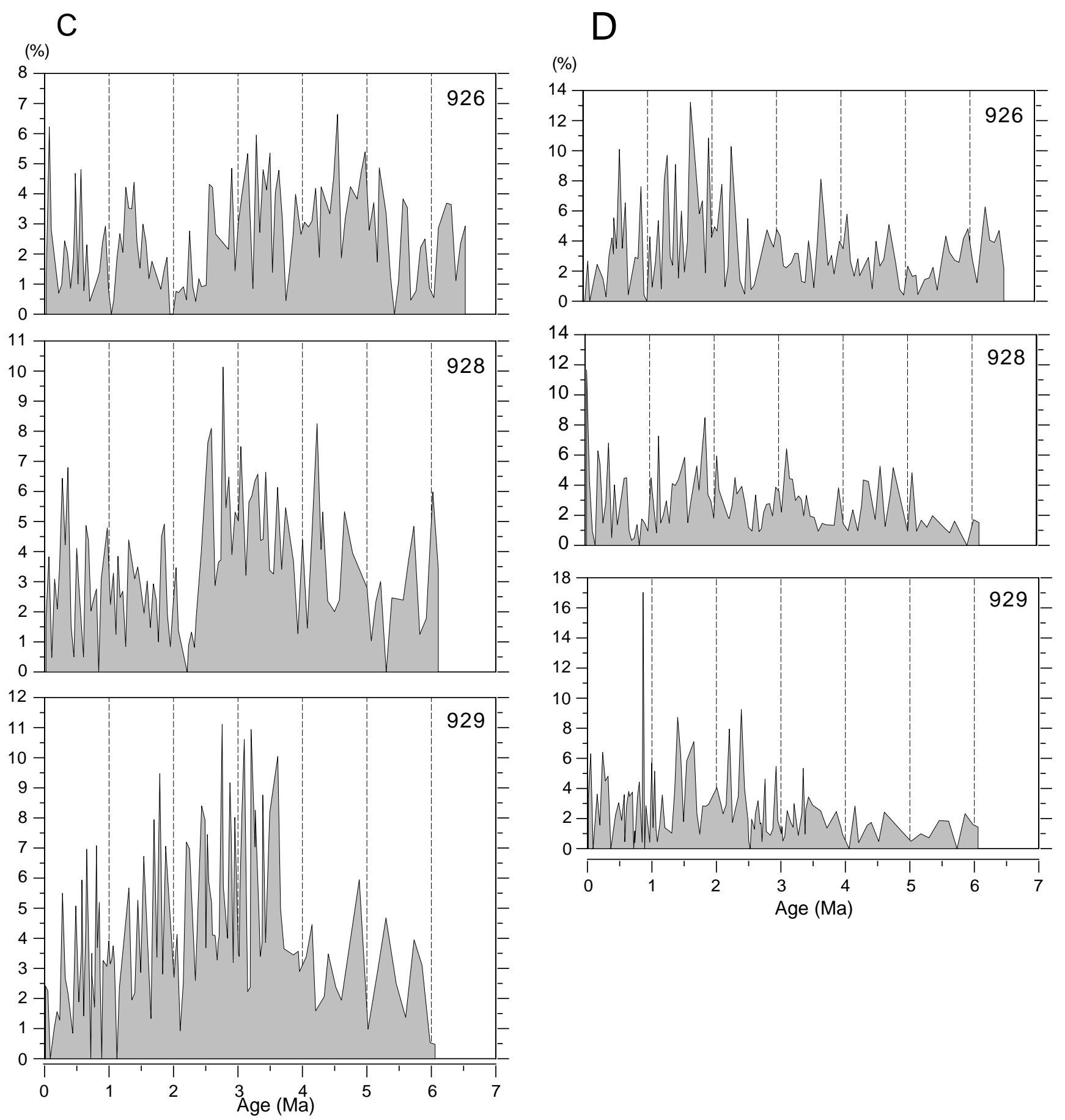

Figure 8 (continued). 

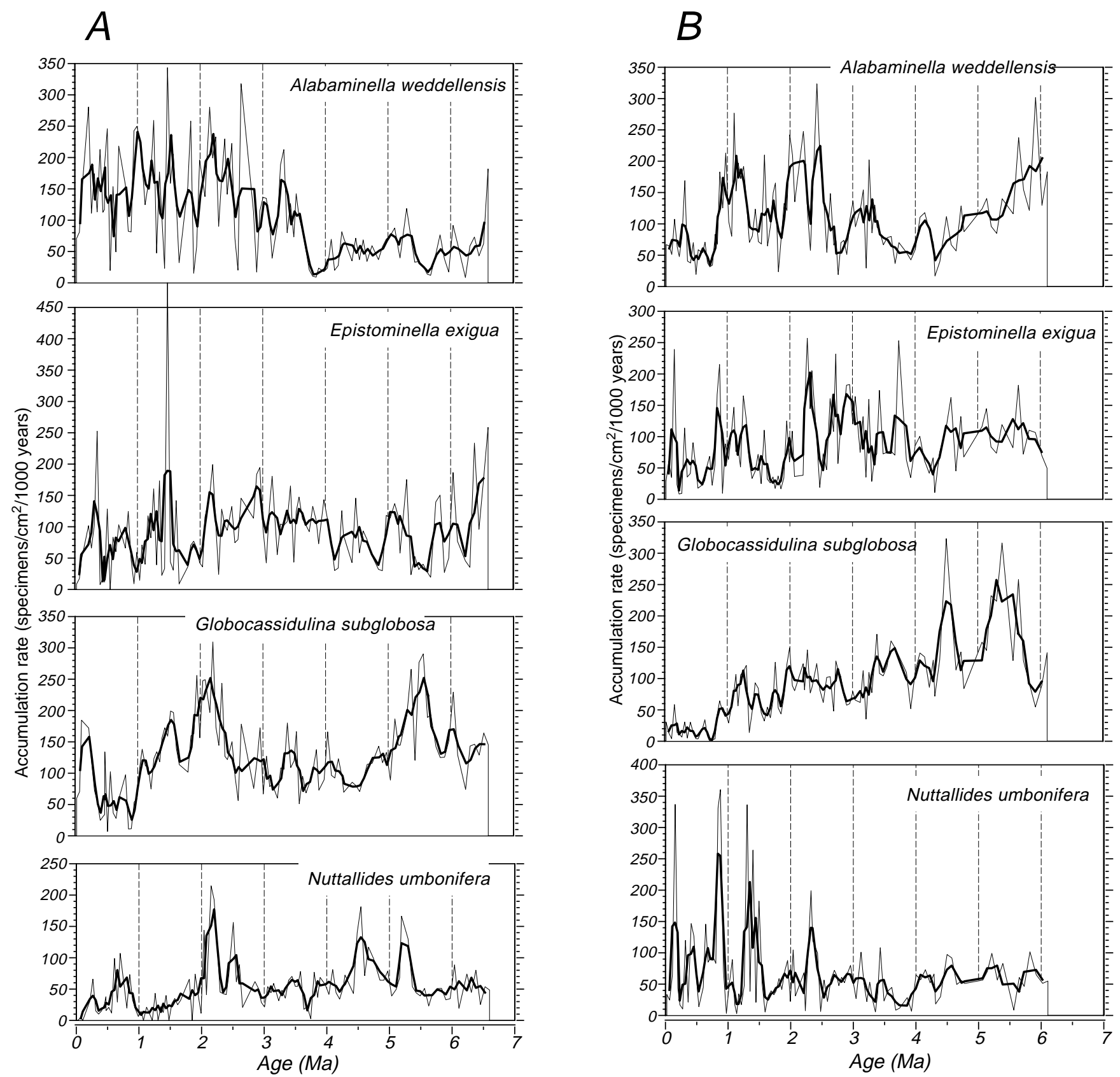

Figure 9. Accumulation rate area graphs for four species in Holes (A) 926A, (B) 928A, and (C) 929A, with the curves of three-point moving averages superimposed. 

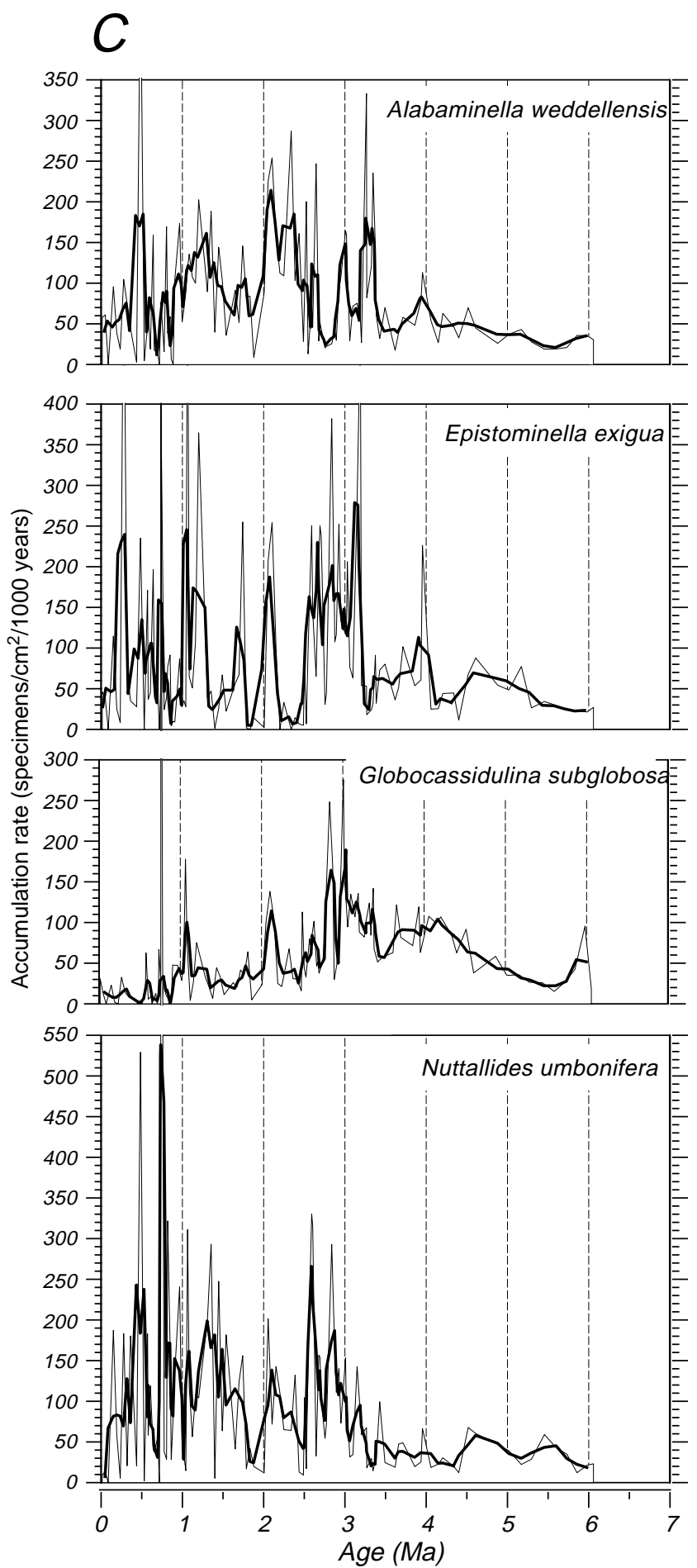

Figure 9 (continued). 

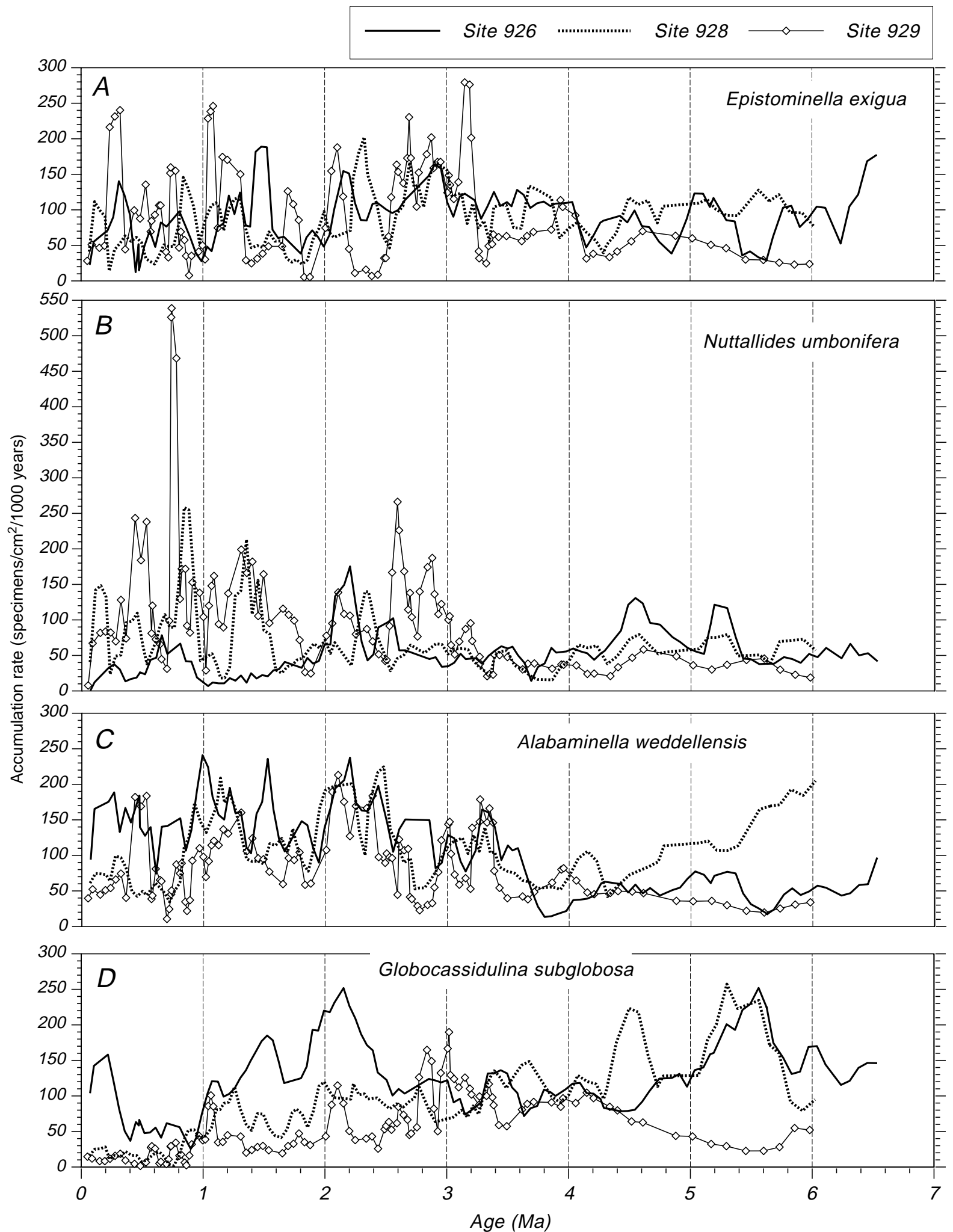

Figure 10. Three-point moving average curves of accumulation rates for four species in Holes 926A, 928A, and 929A. A. Epistominella exigua. B. Nuttallides umbonifera. C. Alabaminella weddellensis. D. Globocassidulina subglobosa. 


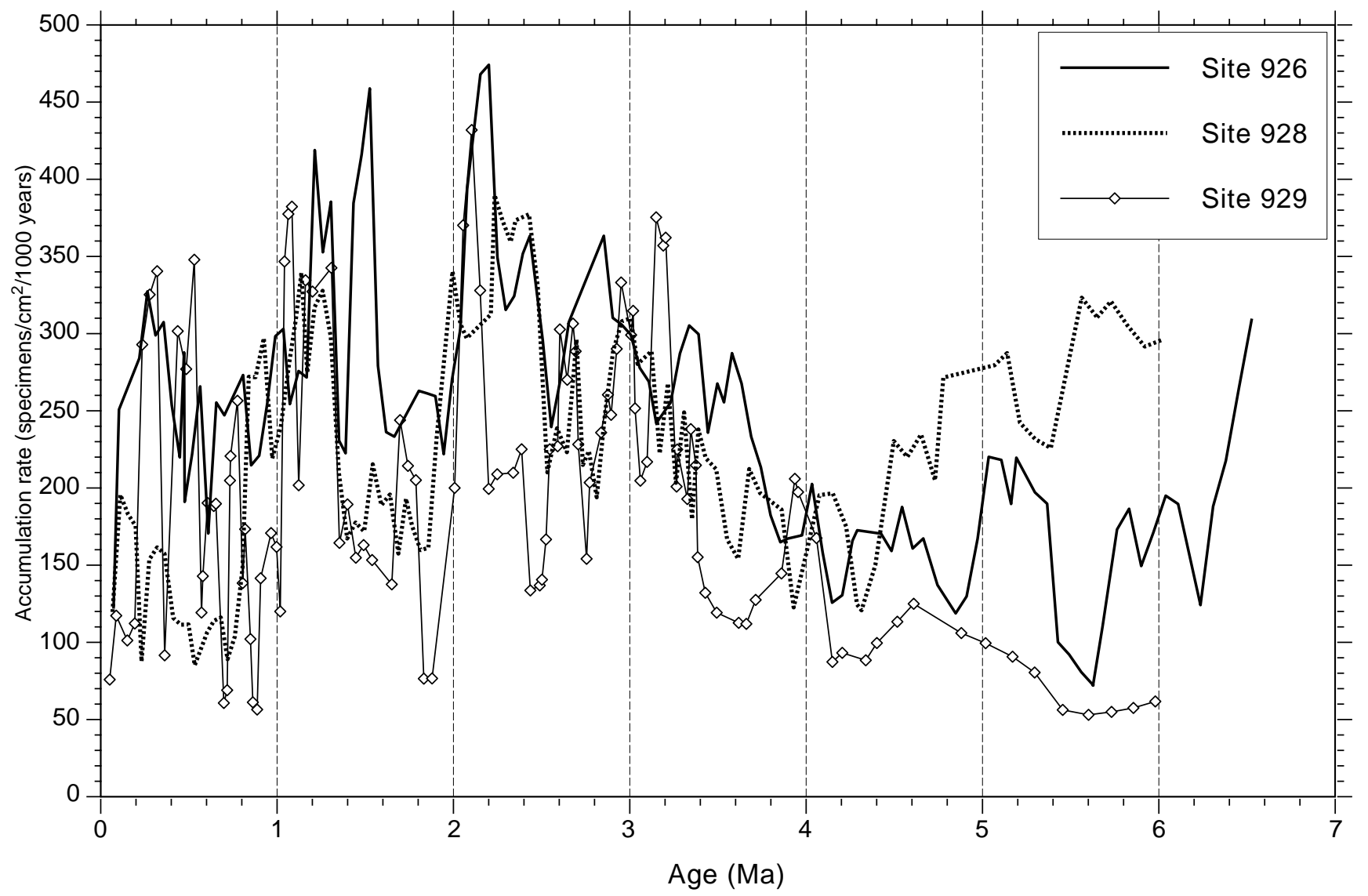

Figure 11. Accumulation rates of phytodetritus species (the sum of Epistominella exigua, Alabaminella weddellensis, Epistominella sp. A, and Ioanella tumidula) in Holes 926A, 928A, and 929A. 


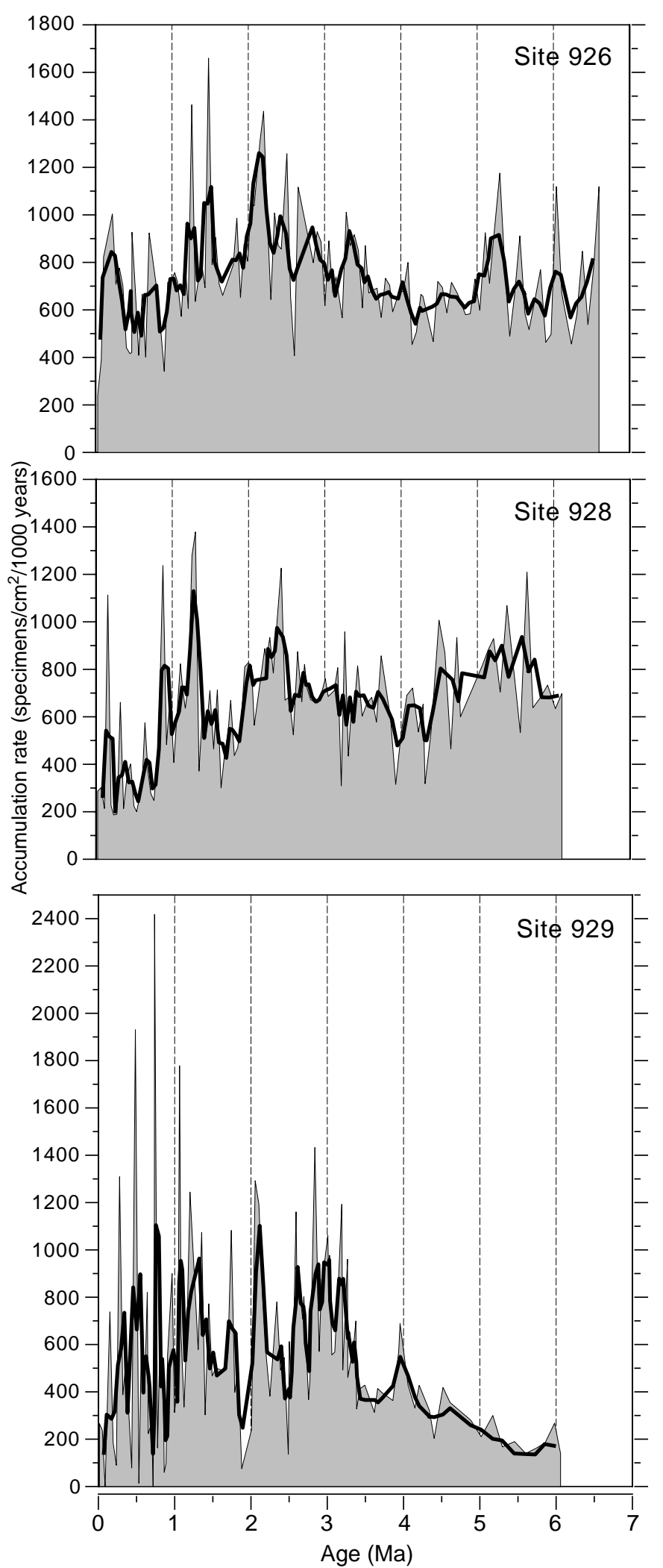

Figure 12. Accumulation rates of total individuals in Holes 926A, 928A, and 929A, with the curves of three-point moving averages superimposed. 


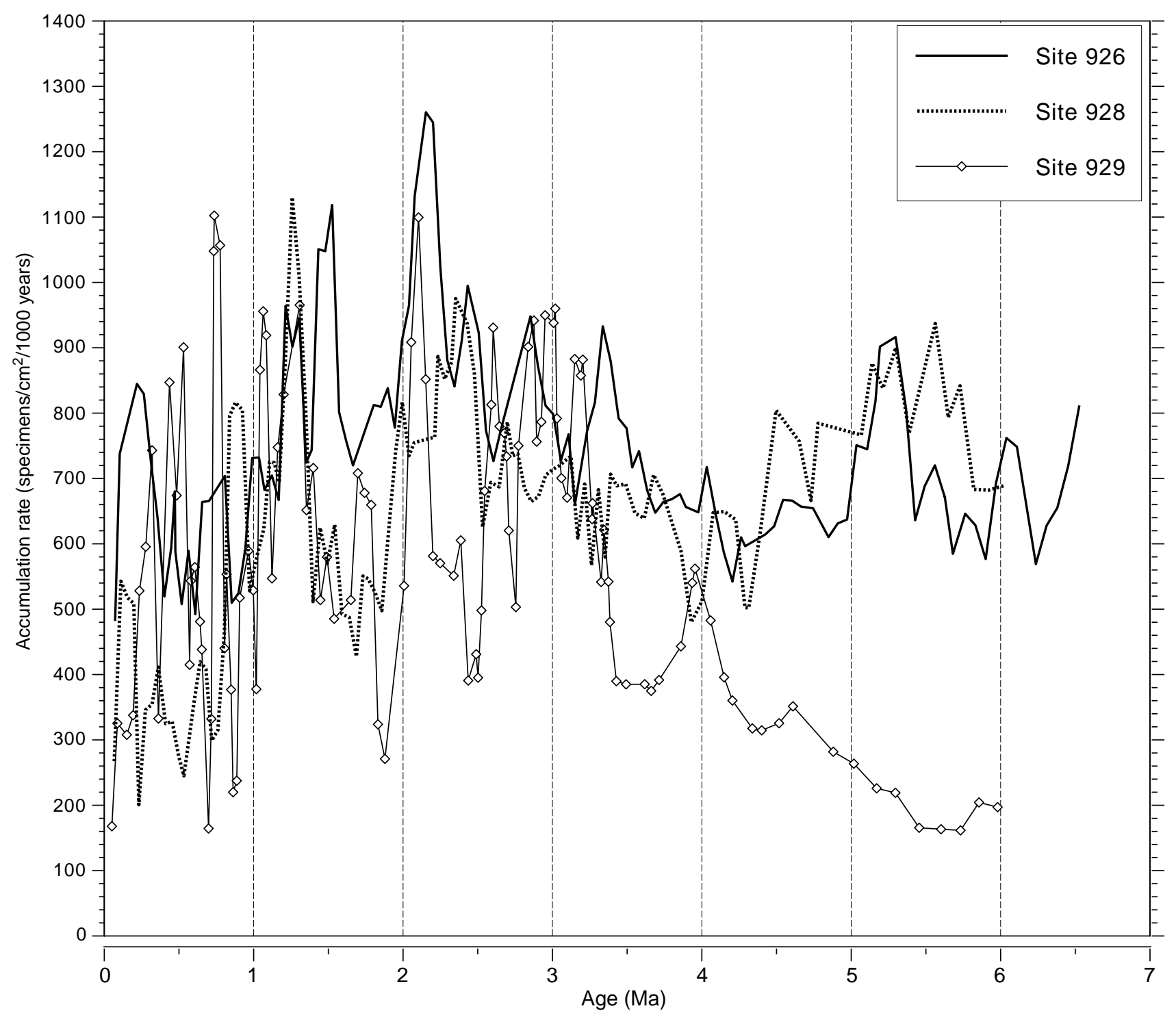

Figure 13. Accumulation rates of total individuals in Holes 926A, 928A, and 929A. 


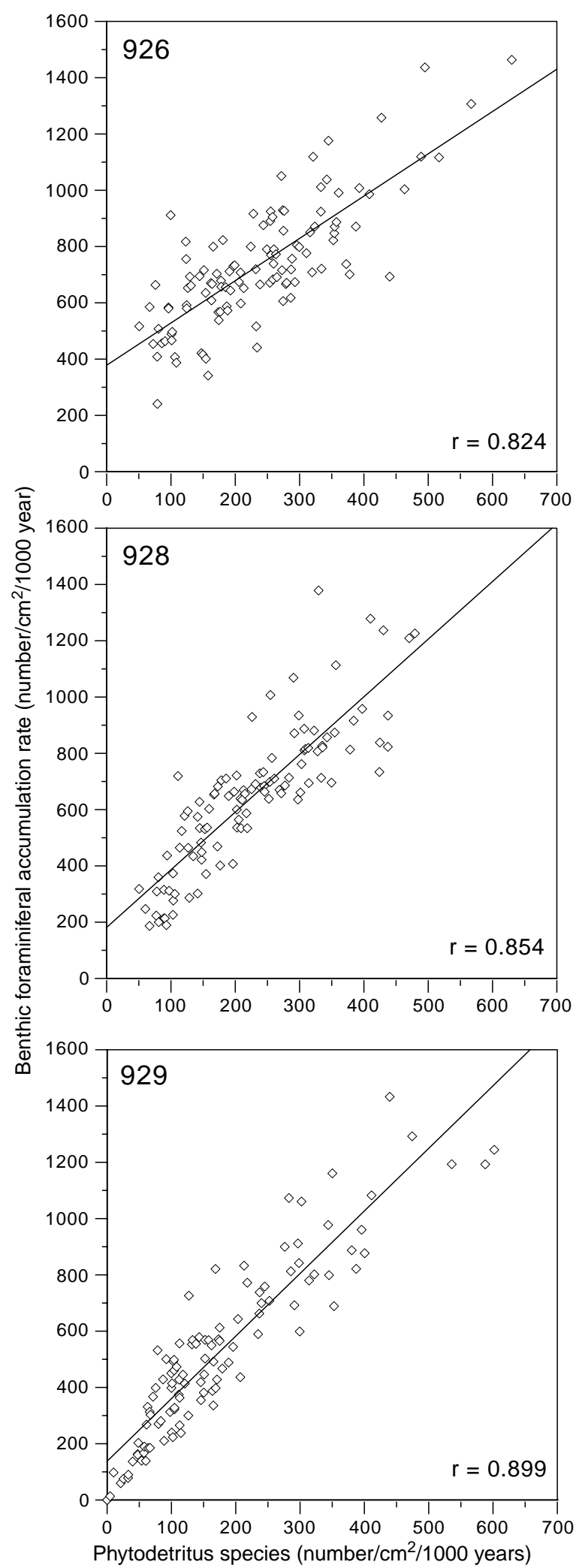

Figure 14. Correlation of accumulation rates between total benthic foraminifers and phytodetritus species in Holes 926A, 928A, and 929A. 
A

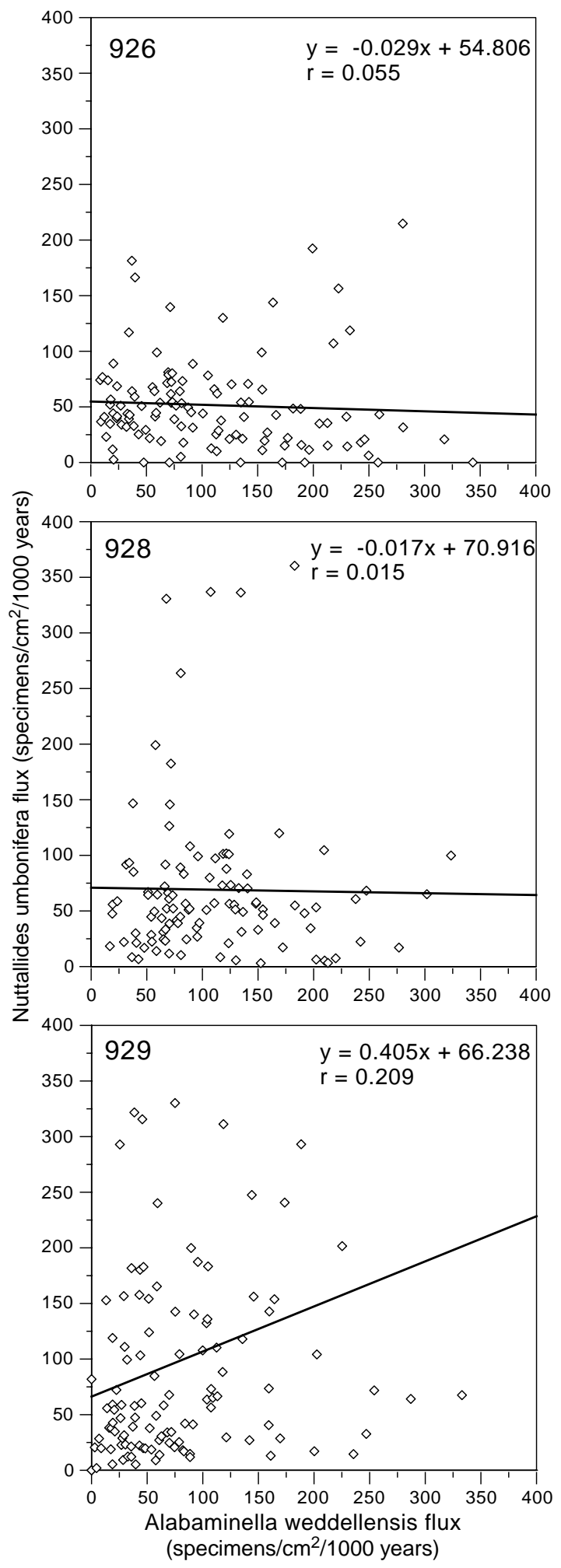

B
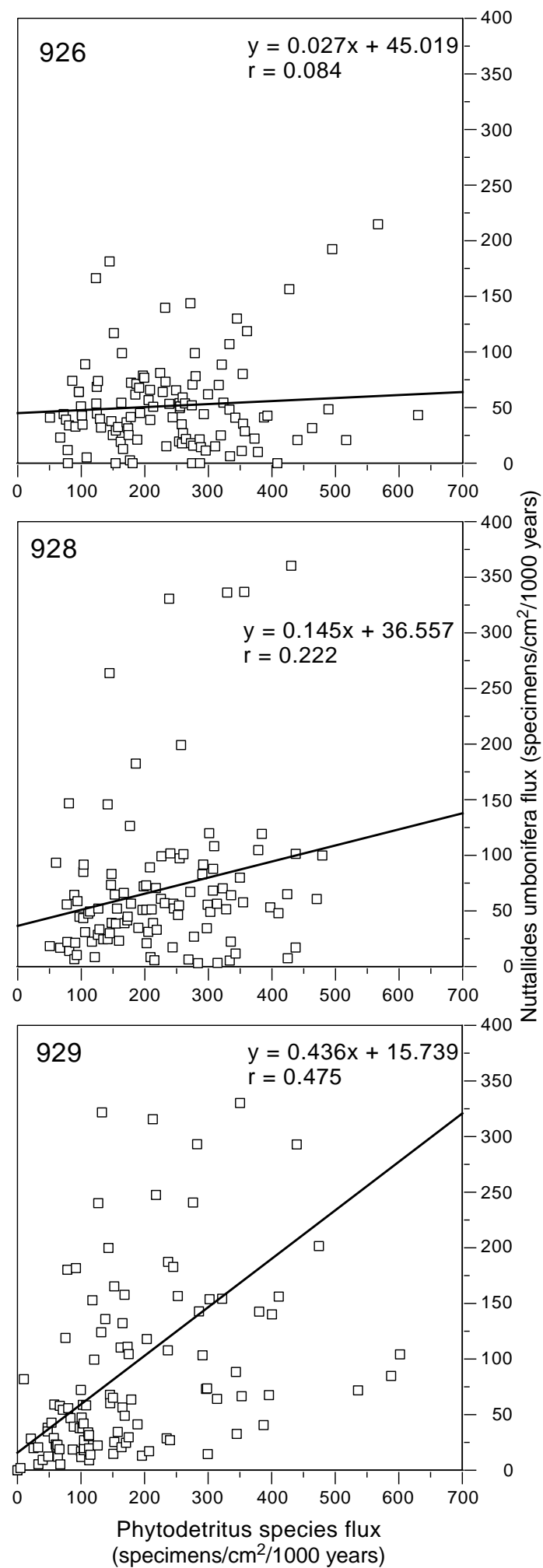

Figure 15. Correlation of accumulation rates between (A) Nuttallides umbonifera and Alabaminella weddellensis, and (B) Nuttallides umbonifera and phytodetritus species in Holes 926A, 928A, and 929A. 

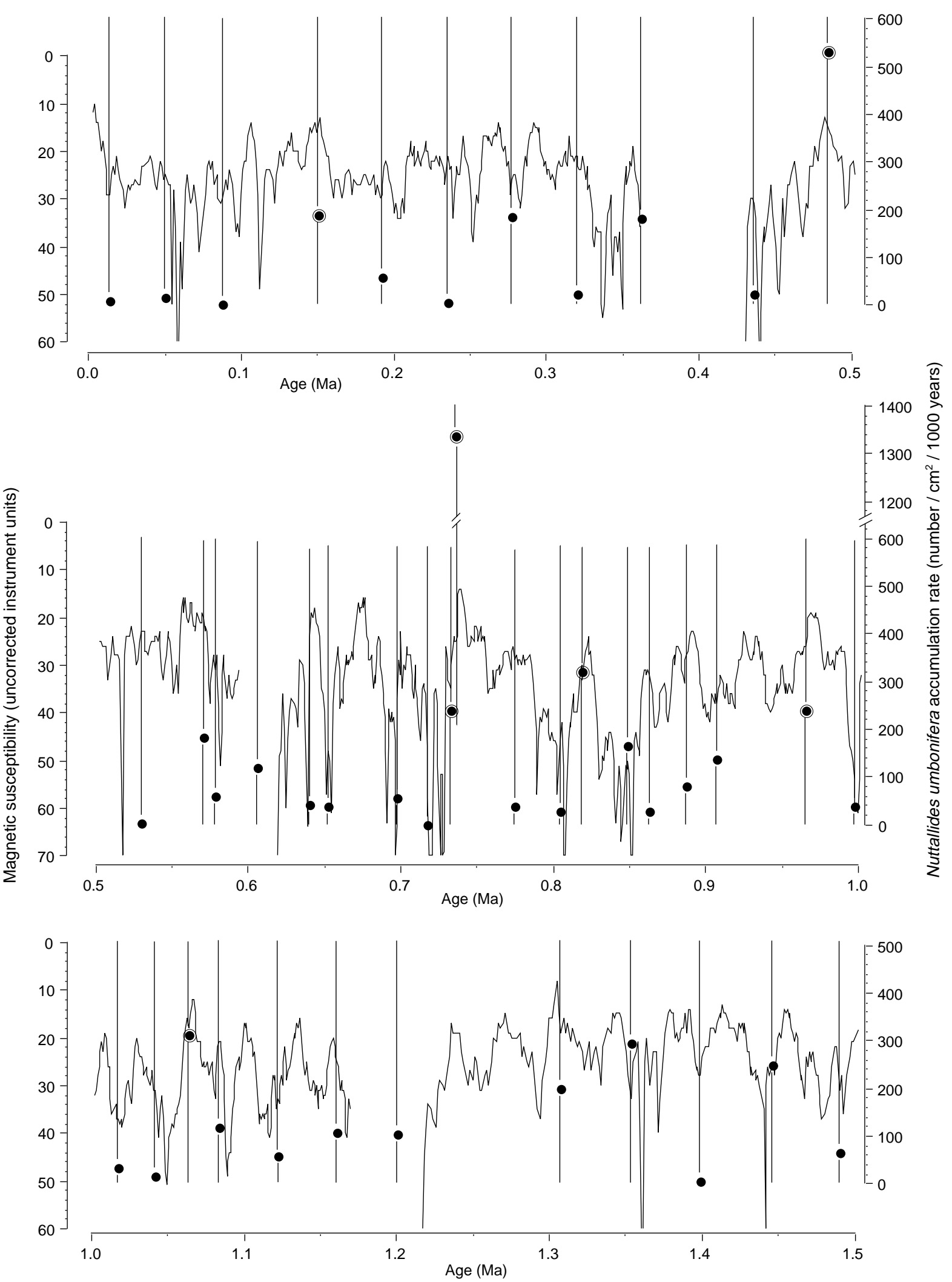

Figure 16. Correlation between magnetic susceptibility records and accumulation rates of Nuttallides umbonifera in Hole 929A during the last 1.5 m.y. Closed circles represent Nuttallides umbonifera accumulation rate values, and closed circles with open circle represent relatively high fluxes of $N$. umbonifera corresponding to low or lowest peaks of magnetic susceptibility. 


\section{APPENDIX}

\section{Faunal Reference List}

Abditodetrix pseudothalmanni (Boltovskoy and Guissani de Khan). Bolivinita pseudothalmanni Boltovskoy and Guissani de Khan, 1981 (fide Loeblich and Tappan, 1988), p. 503, pl. 554, figs. 1-5.

Alabaminella weddellensis (Ealand). Eponides weddellensis Erland, 1936, Discovery Repts., v. 13, p. 57, pl. 1, figs. 65-67.

Anomalinoides globulosus (Chapman and Parr). Anomalina globulosus Chapman and Parr, 1937, Rep. Australasian Antarctic Exped., C, v.1, p. 119, pl. 9, fig. 27.

Astrononion echolsi Kennett, 1967, Contrib. Cushman Lab. Foraminiferal Res., v. 8, p. 408, pl. 11, figs. 14, 15 .

Bolivina pacifica Cushman and McCulloch. Bolivina acerosa Cushman var. pacifica Cushman and McCulloch, 1942, Allan Hancock Pacific Exped., v. 6 , p. 185, pl. 21, figs. 2,3 .

Bulimina alazanensis Cushman, 1927, J. Paleontol., v. 1, p. 161, pl. 25, fig. 4.

Chilostomella oolina Schwager, 1878, Boll. della Reale Comit. Geol. Italiana, v. 9, p. 527, pl. 1, fig. 16.

Cibicidoides bradyi (Trauth). Truncatulina bradyi Trauth, 1884, Denkschr. K. Akad. Wiss. Wien, Math. Naturwiss. Kl., v. 95, p. 235, pl. 4, figs. 7-9.

Cibicidoides kullenbergi (Phleger, Parker and Peirson). Cibicides kullenbergi Phleger, Parker and Peirson, 1953, Rep. Swed. Deep Sea Exped., 19471948 , v. 7, pl. 11, figs. 7, 8 .

Cibicidoides mundulus (Brady, Parker and Jones). Truncatulina mundulus Brady, Parker and Jones, 1888, Trans. Zool. Soc. London, v. 12, p. 228 , pl. 45 , fig. 25a-c.

Cibicidoides robertsonianus (Brady). Planorbulina (Truncatulina) robertsoniana Brady, 1881, Q. J. Microsc. Sci., new ser., v. 21, p. 65.

Cibicidoides wuellerstorfi (Schwager). Anomalina wuellerstorfi Schwager, 1866, Novara Exp. Geol. Theil., v. 2, p. 258, pl. 7, figs. 105, 107.

Discorbinella bertheloti (d'Orbigny). Rosalina bertheloti d'Orbigny, 1839, Historie naturelle des iles Canaries, v. 2, p. 135, pl. 1, figs. 28-30.

Epistominella exigua (Brady). Pulvinulina exigua Brady, 1884, Rep. Voy. Challenger, Zool., v. 9, p. 696, pl. 103, figs. 13, 14.

Globocassidulina subglobosa (Brady). Cassidulina subglobosa Brady, 1884, Rep. Voy. Challenger, Zool., v. 9, p. 430, pl. 54, figs. 17a-c.

Gyroidina lamarckianus (d'Orbigny). Rotalina lamarckiana d'Orbigny, 1839, Hist. Nat. Iles Canaries, v. 2, p. 131, pl. 2, figs. 13-15.

Gyroidina regularis (Phleger and Parker). Eponides regularis Phleger and Parker, 1951, Mem. Geol. Soc. Am., v. 46, pt. 2, p. 21, pl. 11, figs. 3a, b, $4 \mathrm{a}-\mathrm{c}$.

Gyroidina umbonata (Silvestri). Rotalia soldanii d'Orbigny var. umbonata Silvestri, 1898, Accad. Pont. Nuovi Lincei, Mem., vol. 15, p. 329, pl. 6, figs. $14 a-c$.
Gyroidinoides neosoldanii (Brotzen). Gyroidina neosoldanii Brotzen, 1936, Sweden, Sver. Geol. Unders. Avh., Ser. C, no. 396, p. 158.

Gyroidinoides orbicularis (d'Orbigny). Gyroidina orbicularis d'Orbigny, 1826, Ann. Sci. Nat., ser. 1, p. 278.

Ioanella tumidula (Brady). Truncatulina tumidula Brady, 1884, Rep. Voy. Challenger, Zool., v. 9, p. 666, pl. 95, figs. 8a-c.

Laticarinina pauperata (Perker and Jones). Pulvinulina repanda Fichtel and Moll, var. menardii d'Orbigny, subvar. pauperata Perker and Jones, 1865, Philos. Trans. R. Soc. London, v. 155, p. 395, pl. 16, figs. 50, 51a-c.

Melonis barleeanus (Williamson). Nonionina barleeana Williamson, 1858, Recent Foraminifera of Great Britain, p. 32, pl. 3, figs. 68, 69.

Melonis pompilioides (Fichtel and Moll). Nautilus pompilioides Fichtel and Moll, 1798, Test. Microsc., p. 31, pl. 2, figs. a-c.

Nuttallides umbonifera (Cushman). Pulvinulina umbonifera Cushman, 1933, Contrib. Cushman Lab. Foraminiferal Res., v. 9, pt. 4, p. 90, pl. 9, figs. $9 \mathrm{a}-\mathrm{c}$.

Oridorsalis tener (Brady). Truncatulina tenera Brady, 1884, Rep. Voy. Challenger, Zool., v. 9, p. 665, pl. 95, figs. 11a-c.

Oridorsalis umbonatus (Reuss). Rotalina umbonata Reuss, 1851, Z. Dtsch. Geol. Ges., v. 3, pl. 5, figs. 35a-c.

Pullenia bulloides (d'Orbigny). Nonionina bulloides d'Orbigny, 1846, Foraminifères fossiles du Bassin Tertiaire de Vienne, p. 107, pl. 5, figs. 9, 10.

Pullenia osloensis Feyling-Hanssen, 1954, Norsk Geol. Tidskrift, v. 33, no. 12, p. 133, pl. 2, fig. 3a, b.

Pullenia quinqueloba (Reuss). Nonionina quinqueloba Reuss, 1851, Z. Dtsch. Geol. Ges., v. 3, p. 47, pl. 5, fig. 31.

Pullenia zaandamae (van Voorthuysen). Anomalinoides barleeanum (Williamson) var. zaandamae van Voorthuysen, 1952, J. Paleontol., v. 26, no. 4, p. 680-681.

Pyrgo murrhina (Schwager). Biloculina murrhina Schwager, 1866, Novara Exp. Geol. Theil., v. 2, p. 203, pl. 4, figs. 15a-c.

Siphotextularia catenata (Cushman). Textularia catenata Cushman, 1911, Bull. U. S. Nat. Mus., v. 71, p. 23, figs. 39-40.

Sphaeroidina bulloides d'Orbigny, 1826, Ann. Sci. Nat., v. 7, p. 267, modèles no. 65 .

Stainforthia fusiformis (Williamson). Bulimina pupoides d'Orbigny var. fusiformis Williamson, 1858, Roy. Soc., London, p. 63, pl. 5, figs. 129, 130.

Tosaia hanzawai Takayanagi, 1953, Tohoku Univ. Inst. Geol. Paleont., Short Papers, no. 5, p. 30, pl. 4, figs. 7a, b.

Uvigerina hispida Schwager, 1866, Geol. Teil, v. 2, pt. 2, p. 249, pl. 7, fig. 95.

Uvigerina peregrina Cushman, 1923, Bull. U. S. Nat. Mus., v. 104, pt. 4, p. 166, pl. 42, figs. 7-10.

Uvigerina proboscidea Schwager, 1866, Geol. Teil, v. 2, pt. 2, p. 250, pl. 7, fig. 96. 